\title{
A Unified Approach for Network Information
}

\section{Theory}

\author{
Si-Hyeon Lee, Member, IEEE, and Sae-Young Chung, Senior Member, IEEE
}

\begin{abstract}
In this paper, we take a unified approach for network information theory and prove a coding theorem, which can recover most of the achievability results in network information theory that are based on random coding. The final single-letter expression has a very simple form, which was made possible by many novel elements such as a unified framework that represents various network problems in a simple and unified way, a unified coding strategy that consists of a few basic ingredients but can emulate many known coding techniques if needed, and new proof techniques beyond the use of standard covering and packing lemmas. For example, in our framework, sources, channels, states and side information are treated in a unified way and various constraints such as cost and distortion constraints are unified as a single joint-typicality constraint.

Our theorem can be useful in proving many new achievability results easily and in some cases gives simpler rate expressions than those obtained using conventional approaches. Furthermore, our unified coding can strictly outperform existing schemes. For example, we obtain a generalized decode-compressamplify-and-forward bound as a simple corollary of our main theorem and show it strictly outperforms previously known coding schemes. Using our unified framework, we formally define and characterize three types of network duality based on channel input-output reversal and network flow reversal combined with packing-covering duality.
\end{abstract}

\section{INTRODUCTION}

In network information theory, we study the fundamental limits of information flow and processing in a network and develop coding strategies that can approach the limits closely. Instead of studying

S.-H. Lee is with the Department of Electrical and Computer Engineering, University of Toronto, Toronto, Canada (e-mail: sihyeon.lee@utoronto.ca). This work was done when she was at KAIST. S.-Y. Chung is with the Department of Electrical Engineering, KAIST, Daejeon, South Korea (e-mail: sychung@ee.kaist.ac.kr). The material in this paper will be presented in part at IEEE ISIT 2015 [1]. 
a fully general network, however, we often study simple canonical models such as the multiple-access channel [2], relay channel [3], and distributed source coding [4] because they are easier to study and more importantly because we can get useful insights from studying them. Once such insights are obtained, one can try to develop a more general theory that is applicable to general networks.

However, such a task is challenging and only partial results have been known so far [5]-[15], in which network model and/or applied coding technique is limited. For example, network coding [5] and compress-and-forward (CF) [3] were unified as noisy network coding in [8], [9], but does not include decode-and-forward (DF) [3]. DF and partial DF [3] were generalized for single-source multiple-relay single-destination networks [6] and for multicast and broadcast networks [13], [14], respectively. In [10], noisy network coding was combined with network DF [6], but does not allow a relay to perform both partial DF and CF simultaneously. For joint source-channel coding problems, a hybrid analog/digital coding strategy [12] was proposed that recovers and generalizes many previously known results. Such a hybrid coding scheme was applied to some relay networks and was shown to unify both amplify-andforward (AF) [16] and CF [3]. In [15], a novel framework for proving achievability was proposed based on output statistics of random binning and source-channel duality. One important feature of this framework is that the addition of secrecy is free, i.e., once an achievability result is obtained for a network model using this framework, an achievability result with additional secrecy constraint is immediately obtained. We note that [12] and [15] took a bottom-up approach in a sense that achievability results are separately obtained for each of various network models.

In this paper, we take a top-down approach and prove a unified achievability theorem for a general network scenario with arbitrarily many nodes. Our setup is general enough such that any combination of source coding, channel coding, joint source-channel coding, and coding for computing problems can be treated. Our result recovers most of the exiting achievability results in network information theory as long as they are based on random coding. Some examples of known results recovered by our theorem are listed as follows:

- Channel coding: Gelfand-Pinsker coding [17], Marton's inner bound for the broadcast channel [18], Han-Kobayashi inner bound for the interference channel [19], [20], coding for channels with actiondependent states [21], interference decoding for a 3-user interference channel [22], [23], Cover-Leung inner bound for the multiple access channel with feedback [24], a combination of partial DF and $\mathrm{CF}$ for the relay channel [3], network DF [6], noisy network coding [8], [9], short message noisy network coding with a DF option [10], offset encoding for the multiple access relay channel [25].

- Source coding: Slepian-Wolf coding [4], Wyner-Ziv coding [26], Berger-Tung inner bound for 
distributed lossy compression [27], [28], Zhang-Berger inner bound for multiple description coding [29].

- Joint source-channel coding: hybrid coding [12] and all previous results recovered by hybrid coding including sending arbitrarily correlated sources over multiple access channels [30], broadcast channels [31], and interference channels [32], [33].

- Coding for computing: coding for computing [34], cascade coding for computing [35].

In Table \. we compare many approaches that attemped to unify various coding strategies 1

Our theorem can be useful in proving new achievability results easily and in some cases gives simpler rate expressions than those obtained using conventional approaches. Furthermore, our unified coding can strictly outperform existing schemes. To illustrate this, we show that a generalized decode-compressamplify-and-forward bound for acyclic networks can be obtained as a simple corollary of our main theorem and show it strictly outperforms previously known coding schemes. As another special case of our main theorem, we derive a generalized decode-compress-and-forward bound for a discrete memoryless network (DMN) in [36], which recovers both noisy network coding [9] and distributed decode-andforward [13] bounds. This is the first time the partial-decode-compress-and-forward bound (Theorem 7) by Cover and El Gamal [3] is generalized for DMN's such that each relay performs both partial DF and CF simultaneously.

Our unified coding theorem enables us to state various types of duality arising in network information theory. Specifically, we formally define and characterize three types of network duality based on channel input-output reversal and network flow reversal combined with packing-covering duality. Our duality results include as special cases many known duality relationships in network information theory, e.g., the duality between coding for multiple-access channel [2] and distributed sources [27], [28] (type-I duality), the duality between Gelfand-Pinsker coding [17] and Wyner-Ziv coding [26] (type-II duality), and the duality between coding for multiple-access channel [2] and broadast channel [18] (type-III duality).

Our unified achievability result is enabled by many novel elements such as a unified framework that represents various network problems in a simple and unified way, a unified coding strategy that consists of a few basic ingredients but can emulate known coding techniques if needed, and new proof techniques beyond the use of standard covering and packing lemmas. In our framework, sources, channels, states and side information are treated in a unified way and various constraints such as cost and distortion

\footnotetext{
${ }^{1}$ The check mark ' $\checkmark$ ' means that the corresponding unification approach subsumes both the model and the achievability bound of previous result.
} 
TABLE I

COMPARISON OF APPROACHES THAT ATTEMPTED UNIFICATION OF VARIOUS NETWORK MODELS AND CODING STRATEGIES

\begin{tabular}{|c|c|c|c|c|c|}
\hline Previous Results & SWC [8] & DDF [13], [14] & NNC-DF [10] & $\mathrm{HC}$ & Our result \\
\hline Gelfand-Pinsker coding [17] & & & & $\checkmark$ & $\checkmark$ \\
\hline Marton coding [18] & & $\checkmark$ & & $\checkmark$ & $\checkmark$ \\
\hline Han-Kobayashi coding [19] & & & & $\checkmark$ & $\checkmark$ \\
\hline Interference decoding [22] & & & & & $\checkmark$ \\
\hline Cover-Leung coding [24] & & & & $\checkmark$ & $\checkmark$ \\
\hline DF [3] & & $\checkmark$ & $\checkmark$ & & $\checkmark$ \\
\hline Partial DF [3] & & $\checkmark$ & & & $\checkmark$ \\
\hline $\mathrm{AF}$ [16] & & & & $\checkmark$ & $\checkmark$ \\
\hline $\mathrm{CF}$ [3] & $\checkmark$ & & $\checkmark$ & $\checkmark$ & $\checkmark$ \\
\hline Combination of partial DF and CF [3] & & & & & $\checkmark$ \\
\hline Network coding [5] & $\checkmark$ & $\checkmark$ & $\checkmark$ & $\checkmark$ & $\checkmark$ \\
\hline NNC [8], [9] & $\checkmark$ & & $\checkmark$ & & $\checkmark$ \\
\hline Wyner-Ziv coding [26] & & & & $\checkmark$ & $\checkmark$ \\
\hline Slepian-Wolf coding [4] & $\checkmark$ & & & $\checkmark$ & $\checkmark$ \\
\hline Berger-Tung coding [27], [28] & & & & $\checkmark$ & $\checkmark$ \\
\hline Zhang-Berger coding [29] & & & & $\checkmark$ & $\checkmark$ \\
\hline $\begin{array}{l}\text { Joint source-channel coding over } \\
\text { multiple access channels [30], broadcast channels [31], } \\
\text { and interference channels [32], [33] }\end{array}$ & & & & $\checkmark$ & $\checkmark$ \\
\hline Hybrid coding [12] & & & & $\checkmark$ & $\checkmark$ \\
\hline Coding for computing 34 & & & & & $\checkmark$ \\
\hline Cascade coding for computing [35] & & & & & $\checkmark$ \\
\hline
\end{tabular}

[Abbreviations] SWC: Slepian-Wolf coding over networks, DDF: distributed decode-and-forward, NNC-DF: noisy network coding with a DF option, HC: hybrid coding

constraints are combined as a joint-typicality constraint, which is specified by a single joint distribution. Furthermore, we mainly consider acyclic discrete memoryless networks (ADMN) in this paper, where information flows in an acyclic manner. However, we also show our coding theorem can also be applied to general DMN's by unfolding the network. Graph unfolding was first used in [5] for network coding.

Our coding scheme has four main ingredients, i.e., superposition coding, simultaneous nonunique decoding, simultaneous compression, and symbol-by-symbol mapping. We note that our coding scheme does not explicitly include binning and multicoding, but is still general enough to emulate them if needed. 
Although each of these coding ingredients is not new, these are tweaked and combined in a special way to enable unification of many previous approaches. In our coding scheme, covering codebooks are used to compress information that each node observes and decodes. These covering codebooks are generated to permit superposition coding [37]. Each node operates according to the following three steps. The first step is simultaneous nonunique decoding [20], [38], [39], where a node uniquely decodes some covering codewords of other nodes together with some other covering codewords that do not need to be decoded uniquely. The next step is simultaneous compression, where the node finds covering codewords simultaneously that carry information about a received channel output sequence and decoded codewords. Since we allow general superposition relationship among covering codebooks, a more general analysis beyond multivariate covering lemma [40], [41] is needed. The last step is a symbol-by-symbol mapping from a received channel output sequence and decoded and covered codewords to a channel input sequence. The technique of using a symbol-by-symbol mapping was introduced in [42], which is referred to as the Shannon strategy. Our symbol-by-symbol mapping from all three, i.e., the channel output sequence and decoded and covered codewords, was first used in [43] for a three-node noncausal relay channel. We note that such a use of symbol-by-symbol mapping results in correlation between a channel input sequence and nonchosen covering codewords and thus the standard packing lemma [41] cannot be applied for the error analysis. Such correlation was problematic in many previous works and solved for some simple networks in [12], [43], [44]. Our proof technique completely solves this correlation issue in a fully general network setup.

This paper is organized as follows. In Section II, we present our unified framework. In Section III, we propose a unified coding scheme and present the main theorem of this paper. We also show various examples to illustrate how to utilize our results. In Section IV] we characterize three types of network duality. To demonstrate usefulness of our unified coding theorem, in Section $\mathrm{V}$, we derive a generalized decode-compress-amplify-and-forward bound as a simple corollary of our theorem and show it strictly outperforms previously known coding schemes. In Section VI, we present a unified coding theorem for the Gaussian case. We conclude this paper in Section VII.

The following notations are used throughout the paper.

\section{A. Notation}

For two integers $i$ and $j,[i: j]$ denotes the set $\{i, i+1, \ldots, j\}$. For a set $S$ of real numbers, $S_{[i]}$ denotes the $i$-th smallest element in $S$ and $S[i]$ denotes $\{j: j \in S, j<i\}$. For constants $u_{1}, \ldots, u_{k}$ and $S \subseteq[1: k], u_{S}$ denotes the vector $\left(u_{j}: j \in S\right)$ and $u_{i}^{j}$ denotes $u_{[i: j]}$ where the subscript is omitted 
when $i=1$, i.e., $u^{j}=u_{[1: j]}$. For random variables $U_{1}, \ldots, U_{k}$ and $S \subseteq[1: k], U_{S}$ and $U_{i}^{j}$ are defined similarly. For sets $T_{1}, \ldots, T_{k}$ and $S \subseteq[1: k], T_{S}$ denotes $\bigcup_{j \in S} T_{j}$ and $T_{i}^{j}$ denotes $T_{[i: j]}$ where the subscript is omitted when $i=1$. Consider two real vectors $u=\left(u_{1}, \ldots, u_{k}\right)$ and $v=\left(v_{1}, \ldots, v_{k}\right)$ of length $k$. We say that $u$ is smaller than $v$ and write $u<v$ if there exists $k^{\prime} \in[1: k]$ such that $u_{j}=v_{j}$ for all $j \in\left[1: k^{\prime}-1\right]$ and $u_{k^{\prime}}<v_{k^{\prime}}$. Furthermore, we say that $u$ is component-wise smaller than $v$ and write $u \prec v$ if $u_{j}<v_{j}$ for all $j \in[1: k]$. $\mathbf{1}$ denotes an all-one vector and $\mathbf{I}$ denotes an identity matrix. When $U$ is a Gaussian random vector with mean $\mu$ and covariance matrix $\Lambda_{U}$, we write $U \sim \mathcal{N}\left(\mu, \Lambda_{U}\right)$. $\mathbb{1}_{u=v}$ is the indicator function, i.e., it is 1 if $u=v$ and 0 otherwise. $\delta(\epsilon)>0$ denotes a function of $\epsilon$ that tends to zero as $\epsilon$ tends to zero.

We follow the notion of typicality in [45], [41]. Let $\pi_{x^{n}}(x)$ denote the number of occurrences of $x \in \mathcal{X}$ in the sequence $x^{n}$. Then, $x^{n}$ is said to be $\epsilon$-typical (or just typical) for $\epsilon>0$ if for every $x \in \mathcal{X}$,

$$
\left|\pi_{x^{n}}(x) / n-p(x)\right| \leq \epsilon p(x) .
$$

The set of all $\epsilon$-typical $x^{n}$ is denoted as $\mathcal{T}_{\epsilon}^{(n)}(X)$, which is shortly denoted as $\mathcal{T}_{\epsilon}^{(n)}$. A jointly typical set (or just a typical set) such as $\mathcal{T}_{\epsilon}^{(n)}(X, Y)$ for multiple variables, which will also be denoted as $\mathcal{T}_{\epsilon}^{(n)}$, is naturally defined from the definition of $\mathcal{T}_{\epsilon}^{(n)}(X)$.

\section{UNIFIED FRAMEWORK}

In this section, we build a unified framework for proving the achievability of many network information theory problems including channel coding, source coding, joint source-channel coding, and coding for computing. Let us first construct a unified framework for point-to-point scenarios and then generalize it to general network scenarios.

\section{A. Point-to-point scenarios}

Consider the standard channel coding and source coding problems [46] illustrated in Fig. 1] These two problems can be stated with the following elements: information to be communicated, node interaction and node processing functions, and the definition of achievability. Let us investigate differences between these two coding problems for each element and discuss how we can unify them into a single framework. In the following, $n$ denotes the number of channel uses for channel coding and the number of source symbols for source coding and $R \geq 0$ denotes the rate in each problem.

- Information to be communicated: In channel coding, a message $I$, uniformly distributed over $[1$ : $\left.2^{n R}\right]$, is communicated from node 1 to node 2 . In source coding, a discrete memoryless source 


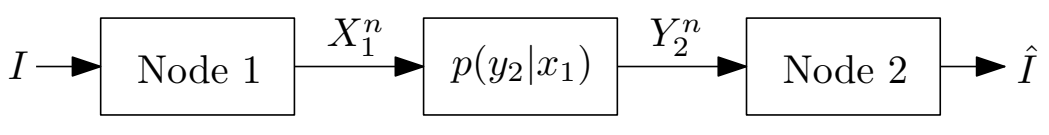

(a)

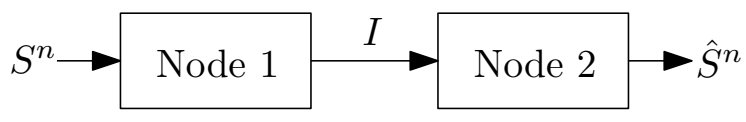

(b)

Fig. 1. (a) Channel coding, (b) Source coding

(DMS) $(\mathcal{S}, p(s))$ is given to node 1 and is reconstructed at node 2 (up to a prescribed distortion level in the case of lossy source coding). We can observe that a message can be regarded as a DMS $(\mathcal{S}, p(s))$ such that $H(S)=R$. Hence, in both channel coding and source coding problems, we can say that a DMS $(\mathcal{S}, p(s))$ is given to node 1 and is reconstructed at node 2 .

- Node interaction and node processing functions: In channel coding, node 1 communicates with node 2 through a discrete memoryless channel (DMC) $\left(\mathcal{X}_{1}, \mathcal{Y}_{2}, p\left(y_{2} \mid x_{1}\right)\right)$. Node 1 maps $s^{n}$ to a channel input sequence $x_{1}^{n}$ and node 2 receives a channel output sequence $y_{2}^{n}$ and maps it to $\hat{s}^{n}$. In source coding, node 1 maps $s^{n}$ to an index $I \in\left[1: 2^{n R}\right]$ and node 2 receives $I$ exactly and maps it to $\hat{s}^{n}$. The noiseless communication of an index in source coding can be regarded as a DMC $\left(\mathcal{X}_{1}, \mathcal{Y}_{2}, p\left(y_{2} \mid x_{1}\right)\right)$ such that $\max _{p\left(x_{1}\right)} I\left(X_{1} ; Y_{2}\right)=R$. Hence, in both channel coding and source coding problems, we can say that node 1 communicates with node 2 through a $\operatorname{DMC}\left(\mathcal{X}_{1}, \mathcal{Y}_{2}, p\left(y_{2} \mid x_{1}\right)\right)$, the processing function at node 1 is a mapping from $S^{n}$ to $X_{1}^{n}$, and the processing function at node 2 is a mapping from $Y_{2}^{n}$ to $\hat{S}^{n}$. By denoting $S$ by $Y_{1}$ and $\hat{S}$ by $X_{2}$, we can further unify the notation for sequences and the node processing functions, i.e., a sequence received by node $k$ is denoted by $Y_{k}^{n}$, the resultant sequence from processing at node $k$ is denoted by $X_{k}^{n}$, and the node processing function at node $k=1,2$ is a mapping from $Y_{k}^{n}$ to $X_{k}^{n}$.

- Achievability: In channel coding and lossless source coding problems, a rate $R$ is said to be achievable if there exists a sequence of node processing functions such that $\lim _{n \rightarrow \infty} P_{e}^{(n)}=0$, where $P_{e}^{(n)}$ denotes the probability of error event given as $P\left(Y_{1}^{n} \neq X_{2}^{n}\right)$. In lossy source coding problem, a rate-distortion pair $(R, d)$ is said to be achievable if there exists a sequence of node processing functions such that $\limsup _{n \rightarrow \infty} \mathrm{E}\left(d\left(Y_{1}^{n}, X_{2}^{n}\right)\right) \leq d$, where $d(\cdot, \cdot) \geq 0$ is a distortion measure between two arguments.

Now, let us introduce a new definition of achievability from which we can show the achievability 
of both channel coding and source coding problems in a unified way. We say a joint distribution $p^{*}\left(x_{1}, x_{2}, y_{1}, y_{2}\right)$, shortly denoted as $p^{*}$, is achievable if there exists a sequence of node processing functions such that $\lim _{n \rightarrow \infty} P_{e}^{(n)}\left(p^{*}, \epsilon\right)=0$ for any $\epsilon>0$, where $P_{e}^{(n)}\left(p^{*}, \epsilon\right)$ denotes the probability

$$
P\left(\left(X_{1}^{n}, X_{2}^{n}, Y_{1}^{n}, Y_{2}^{n}\right) \notin T_{\epsilon}^{(n)}\right)
$$

in which the typical set is defined with respect to $p^{*}$. Then, the achievability of appropriately chosen $p^{*}$ implies the achievability of $R$ or $(R, d)$ in channel coding and source coding problems. For channel coding and lossless source coding problems, $R$ is achievable if $p^{*}$ such that $X_{2}=Y_{1}$ is achievable. For lossy source coding problem, $(R, d)$ is achievable if $p^{*}$ such that $E\left(d\left(Y_{1}, X_{2}\right)\right) \leq d /\left(1+\epsilon^{\prime}\right)$, $\epsilon^{\prime} \rightarrow 0$ is achievable from the typical average lemma [41] and the continuity of the rate-distortion function $R(d)$ in $d$.

To see whether the aforementioned unification approach is general enough for point-to-point scenarios, let us consider more general point-to-point scenarios in Fig. 2 First, in channels with noncausal states [17] illustrated in Fig. 2f(a), node 1 observes a message $I$ of rate $R$ and a state sequence $S^{n} \sim p(s)$ and encodes $\left(I, S^{n}\right)$ as $X_{1}^{n}$. Then, node 2 receives $Y_{2}^{n} \sim p\left(y_{2} \mid s, x_{1}\right)$ and estimates $I$ as $\hat{I}$. Achievability is defined in the same way as in the channel coding problem. Let us apply the aforementioned unification approach to this problem. Since $Y_{1}$ represents all the information node 1 receives, we let $Y_{1}=(M, S)$ such that $H(M)=R$ and $M$ and $S$ are independent, where $M^{n}$ corresponds to the message of rate $R$. But, we cannot use the channel form of $p\left(y_{2} \mid x_{1}\right)$ to capture the dependency of the channel output $Y_{2}$ on state $S$. This indicates that a more general channel form of $p\left(y_{2} \mid y_{1}, x_{1}\right)$ is needed in the unified framework. Then, we can let $p\left(y_{2} \mid y_{1}, x_{1}\right)$ be equal to $p\left(y_{2} \mid s, x_{1}\right)$. If we choose $p^{*}$ such that $X_{2}=M$, the achievability of $p^{*}$ implies the achievability of $R$ of the original problem.

Next, in lossy source coding with side information [26] represented in Fig. 2-(b), node 1 receives a source sequence $S^{n} \sim p(s)$ and encodes it as an index $I \in\left[1: 2^{n R}\right]$. Then, node 2 receives the index $I$ and side information $T^{n} \sim p(t \mid s)$ and reconstructs $S^{n}$ as $\hat{S}^{n}$ up to some distortion level. Achievability is defined in the same way as in the lossy source coding problem. For this problem, we apply the unification approach as follows. We let $Y_{1}=S$. Since node 2 has two channel inputs, we let $Y_{2}=\left(Y_{2}^{\prime}, T\right)$ and let the channel $p\left(y_{2} \mid y_{1}, x_{1}\right)$ be decomposed as $p\left(y_{2}^{\prime} \mid x_{1}\right) p\left(t \mid y_{1}\right)$, where the channel $p\left(y_{2}^{\prime} \mid x_{1}\right)$ corresponds to the communication of $I$ of rate $R$ and hence its capacity is given as $R$, i.e., $\max _{p\left(x_{1}\right)} I\left(X_{1} ; Y_{2}^{\prime}\right)=R$, and the channel $p\left(t \mid y_{1}\right)$ captures the correlation between $Y_{1}=S$ and the side information $T$. We pick up the target distribution in the same way as in the lossy source coding problem. Furthermore, coding for computing problem [34], where node 2 wishes to reconstruct a function $f(S, T)$ of $S$ and $T$ up 


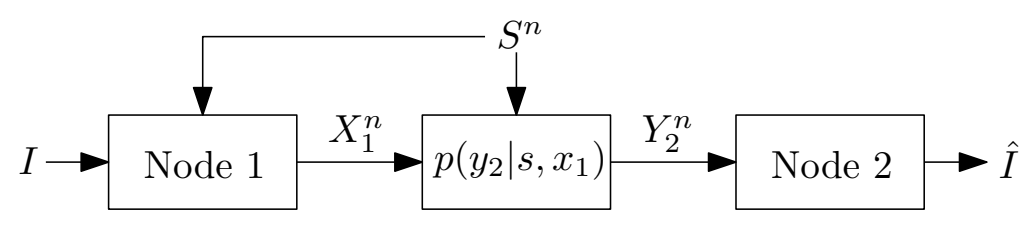

(a)

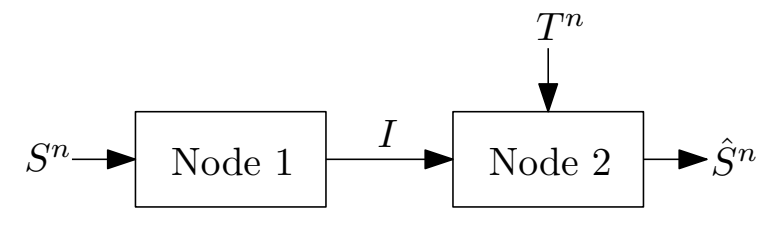

(b)

Fig. 2. (a) Channel with noncausal states, (b) Lossy source coding with side information

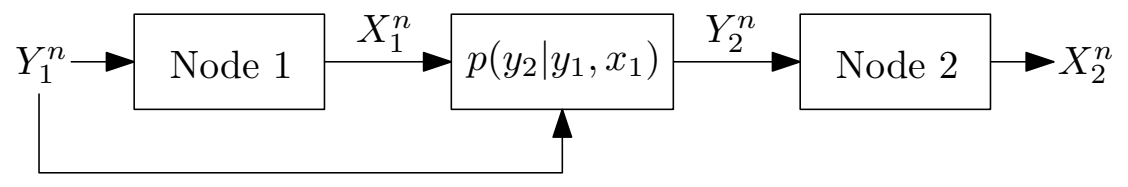

Fig. 3. Unified framework for point-to-point scenarios

to distortion $d$ with respect to a distortion measure $d(\cdot, \cdot)$, can also be included in this framework by choosing $p^{*}$ such that $\mathrm{E}\left(d\left(g\left(Y_{1}, Y_{2}\right), X_{2}\right)\right) \leq d /(1+\epsilon), \epsilon \rightarrow 0$ where $g\left(Y_{1}, Y_{2}\right)=g\left(S, Y_{2}^{\prime}, T\right)=f(S, T)$.

In summary, the achievability of the aforementioned point-to-point coding problems can be shown by considering the following unified framework. Network model is given by $\left(\mathcal{X}_{1}, \mathcal{X}_{2}, \mathcal{Y}_{1}, \mathcal{Y}_{2}, p\left(y_{1}\right) p\left(y_{2} \mid y_{1}, x_{1}\right)\right)$ as illustrated in Fig. 3 and the objective is specified by a target distribution $p^{*} . p^{*}$ is said to be achievable if there exists a sequence of node processing functions, $Y_{k}^{n} \rightarrow X_{k}^{n}, k=1,2$, such that $\lim _{n \rightarrow \infty} P_{e}^{(n)}\left(p^{*}, \epsilon\right)=0$ for any $\epsilon>0$.

\section{B. General scenarios}

In this subsection, we generalize the unified framework in Section $\llbracket-\mathrm{A}$ to general $N$-node networks. In our unified framework for $N$ nodes, we define an $N$-node acyclic discrete memoryless network (ADMN) $\left(\mathcal{X}_{1}, \ldots, \mathcal{X}_{N}, \mathcal{Y}_{1}, \ldots, \mathcal{Y}_{N}, \prod_{k=1}^{N} p\left(y_{k} \mid y^{k-1}, x^{k-1}\right)\right)$, which consists of a set of alphabet pairs $\left(\mathcal{X}_{k}, \mathcal{Y}_{k}\right)$, $k \in[1: N]$ and a collection of conditional pmfs $p\left(y_{k} \mid y^{k-1}, x^{k-1}\right), k \in[1: N]$. Here, $Y_{k}$ and $X_{k}$ represent any information that comes into and goes out of node $k$, respectively. $Y_{k}$ can be a channel output, message, source, non-causal state information, and any combination of those. $X_{k}$ can be a channel input, message estimate, reconstructed source, action for generating channel state, and any combination of those. Next, 
$p\left(y_{k} \mid y^{k-1}, x^{k-1}\right)$ signifies the correlation between information prior to node $k$ and information received at node $k$. It can capture channel distribution possibly with states, correlation between distributed sources, and complicated network-wide correlation among sources and channels.

In this network, information flows in one direction and node operations are sequential. Let $n$ denote the number of channel uses. First, $Y_{1}^{n}$ is generated according to $\prod_{i=1}^{n} p\left(y_{1, i}\right)$ and then node 1 processes $X_{1}^{n}$ based on $Y_{1}^{n}$. Next, $Y_{2}^{n}$ is generated according to $\prod_{i=1}^{n} p\left(y_{2, i} \mid x_{1, i}, y_{1, i}\right)$ and then node 2 encodes $X_{2}^{n}$ based on $Y_{2}^{n}$. Similarly, $Y_{k}^{n}$ is generated according to $\prod_{i=1}^{n} p\left(y_{k, i} \mid x_{i}^{k-1}, y_{i}^{k-1}\right)$ and node $k$ encodes $X_{k}^{n}$ based on $Y_{k}^{n}$ for $k \in[1: N]$. Clearly, any layered network [7] or noncausal network (without infinite loop) [47] possibly with noncausal state or side information is represented as an ADMN. Furthermore, any strictly causal (usual discrete memoryless network with relay functions having one sample delay) or causal network (relays without delay [47]) with blockwise operations can be represented as an ADMN by unfolding the network. Note that our unified achievability theorem (Theorem 11) still applies to the unfolded network. Therefore, considering only acyclic DMN (ADMN) in our unified approach is without loss of generality while greatly simplifying our unification approach. In the following subsection, we show several known examples represented by an ADMN.

Achievability is specified using a target joint distribution $p^{*}\left(x^{N}, y^{N}\right)$, which is shortly denoted as $p^{*}$. For a set of node processing functions $Y_{k}^{n} \rightarrow X_{k}^{n}, k=1, \ldots, N$, the $\epsilon$-probability of error is defined as $P_{e}^{(n)}\left(p^{*}, \epsilon\right)=P\left(\left(X_{[1: N]}^{n}, Y_{[1: N]}^{n}\right) \notin \mathcal{T}_{\epsilon}^{(n)}\right)$, where the typical set $\mathcal{T}_{\epsilon}^{(n)}$ is defined with respect to $p^{*}$. We say the target distribution $p^{*}$ is achievable if there exists a sequence of node processing functions $Y_{k}^{n} \rightarrow X_{k}^{n}, k=1, \ldots, N$, such that $\lim _{n \rightarrow \infty} P_{e}^{(n)}\left(p^{*}, \epsilon\right)=0$ for any $\epsilon>0$. We note that $p^{*}$ unifies diverse network demands and constaints. It can be used for designating the source-destination relationship and for imposing distortion and cost constraints.

\section{Examples}

In this subsection, we represent some network information theory problems by an ADMN and a target distribution $p^{*}$ such that the achievability of $p^{*}$ implies the achievability of the original problem. Let us first consider some examples of single-hop networks.

Example 1 (Multiple access channels [2]): For multiple access channel problem with rates $R_{1}$ and $R_{2}$, we choose $N=3, H\left(Y_{1}\right)=R_{1}, p\left(y_{2} \mid x_{1}, y_{1}\right)=p\left(y_{2}\right), H\left(Y_{2}\right)=R_{2}, p\left(y_{3} \mid x_{1}, x_{2}, y_{1}, y_{2}\right)=p\left(y_{3} \mid x_{1}, x_{2}\right)$, and $p^{*}$ such that $X_{3}=\left(Y_{1}, Y_{2}\right)$.

Example 2 (Distributed lossy compression [27], [28]): For distributed lossy compression problem with rate-distortion pairs $\left(R_{1}, d_{1}\right)$ and $\left(R_{2}, d_{2}\right)$, we let $N=3, p\left(y_{2} \mid x_{1}, y_{1}\right)=p\left(y_{2} \mid y_{1}\right), \mathcal{Y}_{3}=\mathcal{Y}_{3,1} \times \mathcal{Y}_{3,2}$, 
$p\left(y_{3} \mid x_{[1: 2]}, y_{[1: 2]}\right)=p\left(y_{3,1} \mid x_{1}\right) p\left(y_{3,2} \mid x_{2}\right)$ such that $\max _{p\left(x_{1}\right)} I\left(X_{1} ; Y_{3,1}\right)=R_{1}$ and $\max _{p\left(x_{2}\right)} I\left(X_{2} ; Y_{3,2}\right)=$ $R_{2}$, and $p^{*}$ such that $\mathrm{E}\left[d_{k}\left(Y_{k}, X_{3}\right)\right] \leq \frac{d_{k}}{1+\epsilon}$ for $k \in[1: 2]$, where $d_{k}(\cdot, \cdot) \geq 0$ is a distortion measure between two arguments and $\epsilon \rightarrow 0$.

Example 3 (Broadcast channels [18]): For broadcast channel problem with rates $R_{1}$ and $R_{2}$, we choose $N=3, Y_{1}=\left(Y_{1,1}, Y_{1,2}\right), p\left(y_{1,1}, y_{1,2}\right)=p\left(y_{1,1}\right) p\left(y_{1,2}\right), H\left(Y_{1,1}\right)=R_{1}, H\left(Y_{1,2}\right)=R_{2}, p\left(y_{2} \mid y_{1}, x_{1}\right)=$ $p\left(y_{2} \mid x_{1}\right), p\left(y_{3} \mid y_{[1: 2]}, x_{[1: 2]}\right)=p\left(y_{3} \mid x_{1}, y_{2}\right)$, and $p^{*}$ such that $X_{2}=Y_{1,1}, X_{3}=Y_{1,2}$.

Example 4 (Multiple description coding [48]): For multiple description coding with rates $\left(R_{1}, R_{2}\right)$ and distortion triples $d_{1}, d_{2}$, and $d_{3}$, we choose $N=4, \mathcal{X}_{1}=\mathcal{X}_{1,1} \times \mathcal{X}_{1,2}, p\left(y_{2} \mid x_{1}, y_{1}\right)=p\left(y_{2} \mid x_{1,1}\right)$ such that $\max _{p\left(x_{1,1}\right)} I\left(X_{1,1} ; Y_{2}\right)=R_{1}, p\left(y_{3} \mid x_{[1: 2]}, y_{[1: 2]}\right)=p\left(y_{3} \mid x_{1,2}\right)$ such that $\max _{p\left(x_{1,2}\right)} I\left(X_{1,2} ; Y_{3}\right)=R_{2}$, $Y_{4}=\left(Y_{2}, Y_{3}\right)$, and $p^{*}$ such that $\mathrm{E}\left[d_{k}\left(Y_{1}, X_{k}\right)\right] \leq \frac{d_{k}}{1+\epsilon}$ for $k \in[2: 4]$, where $d_{k}(\cdot, \cdot) \geq 0$ is a distortion measure between two arguments and $\epsilon \rightarrow 0$.

Next, we show an example of multi-hop networks.

Example 5 (Relay channels): Consider a three-node relay channel $\left(\mathcal{X}_{1}, \mathcal{X}_{2}, \mathcal{Y}_{2}, \mathcal{Y}_{3}, p\left(y_{2}, y_{3} \mid x_{1}, x_{2}\right)\right)$ illustrated in Fig. 4.(a), where node 1 wishes to send a message to node 3 with the help of node 2 . Let $R$ and $n$ denote the rate and the number of channel uses, respectively, and let $I$ and $\hat{I}$ denote the message of rate $R$ at node 1 and the estimated message at node 3 , respectively. Then, the node processing function at node 1 is a mapping from $\left[1: 2^{n R}\right]$ to $\mathcal{X}_{1}^{n}$, the node processing function at node 2 at time $i \in[1: n]$ is a mapping from $\mathcal{Y}_{2}^{i-1}$ to $\mathcal{X}_{2}$, and the node processing function at node 3 is a mapping from $\mathcal{Y}_{3}^{n}$ to $\left[1: 2^{n R}\right]$. The probability of error is defined as $P_{e}^{(n)}=P(\hat{I} \neq I)$ and a rate $R$ is said to be achievable if there exists a sequence of node processing functions such that $\lim _{n \rightarrow \infty} P_{e}^{(n)}=0$.

If we assume a blockwise operation at each node, we can represent this network as an ADMN by unfolding the network. Assume $B$ transmission blocks, each consisting of $n$ channel uses. In the unfolded network illustrated in Fig. 4-(b), we have $3(B+1)$ nodes and the operation of node $(k, b), k \in[1: 3], b \in$ $[1: B+1]$ corresponds to that of node $k$ of the original network at the end of block $b-1$. To reflect the fact that node $(k, b+1)$ is originally the same node as node $(k, b)$, we assume that node $(k, b)$ has an orthogonal link of sufficiently large rate to node $(k, b+1)$, which is represented as a dashed line in Fig. 44(b). Because this unfolded network is acyclic, it can be represented as an ADMN and $p^{*}$ can be chosen accordingly.

\section{Introduction of a virtual node}

The following two propositions are obtained by introducing a virtual node in an ADMN, which turn out to be useful in recovering some known achievability results in Section 


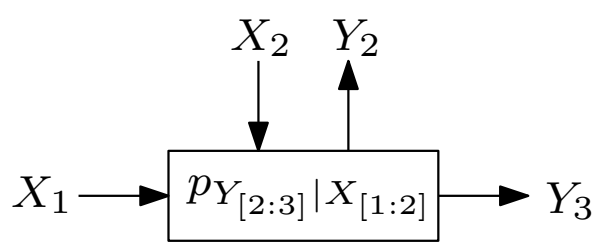

(a)

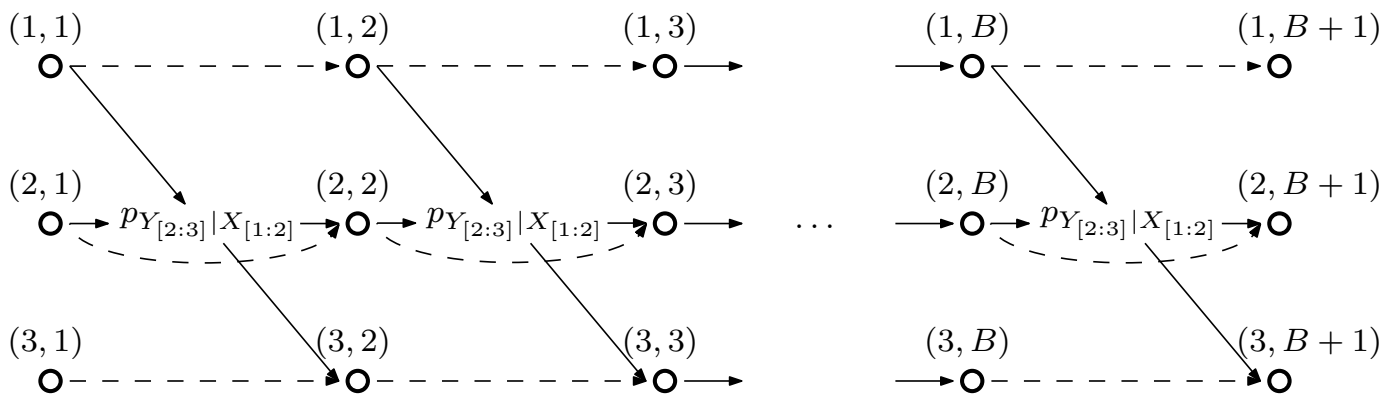

(b)

Fig. 4. Three-node relay network is illustrated in (a). The corresponding unfolded network is shown in (b). In the unfolded network, the operation of node $(k, b)$ corresponds to that of node $k$ of the original network at the end of block $b-1$.

Proposition 1: Consider an $N$-node ADMN

$$
\left(\mathcal{X}_{1}, \ldots, \mathcal{X}_{N}, \mathcal{Y}_{1}, \ldots, \mathcal{Y}_{N}, \prod_{k=1}^{N} p\left(y_{k} \mid y^{k-1}, x^{k-1}\right)\right)
$$

and target distribution $p^{*}$. For some $v \in[1: N]$ and finite set $\mathcal{Y}$, assume $p\left(y \mid x^{v-1}, y^{v}\right)$ for $y \in \mathcal{Y}$. Then, we have

$$
\begin{gathered}
p\left(y \mid x^{v-1}, y^{v-1}\right)=\sum_{y_{v}} p\left(y_{v} \mid x^{v-1}, y^{v-1}\right) p\left(y \mid x^{v-1}, y^{v}\right) \\
p\left(y_{v} \mid x^{v-1}, y^{v-1}, y\right)=\frac{p\left(y_{v} \mid x^{v-1}, y^{v-1}\right) p\left(y \mid x^{v-1}, y^{v}\right)}{\sum_{y_{v}} p\left(y_{v} \mid x^{v-1}, y^{v-1}\right) p\left(y \mid x^{v-1}, y^{v}\right)} .
\end{gathered}
$$

Now, consider an $(N+1)$-node ADMN

$$
\left(\mathcal{X}_{1}^{\prime}, \ldots, \mathcal{X}_{N+1}^{\prime}, \mathcal{Y}_{1}^{\prime}, \ldots, \mathcal{Y}_{N+1}^{\prime}, \prod_{k=1}^{N+1} p^{\prime}\left(y_{k} \mid y^{k-1}, x^{k-1}\right)\right)
$$

and target distribution $p^{*}$ such that

$$
\mathcal{X}_{k}^{\prime}=\left\{\begin{array}{l}
\mathcal{X}_{k} \text { if } k<v \\
\emptyset \text { if } k=v \\
\mathcal{X}_{k-1} \text { if } k>v
\end{array} \quad, \quad \mathcal{Y}_{k}^{\prime}=\left\{\begin{array}{l}
\mathcal{Y}_{k} \text { if } k<v \\
\mathcal{Y} \text { if } k=v \\
\mathcal{Y}_{k-1} \text { if } k>v
\end{array},\right.\right.
$$




$$
p^{\prime}\left(y_{k} \mid x^{k-1}, y^{k-1}\right)=\left\{\begin{array}{l}
p_{Y_{k} \mid X^{k-1}, Y^{k-1}}\left(y_{k} \mid x^{k-1}, y^{k-1}\right) \text { if } k<v \\
p_{Y \mid X^{k-1}, Y^{k-1}}\left(y_{k} \mid x^{k-1}, y^{k-1}\right) \text { if } k=v \\
p_{Y_{v} \mid X^{v-1}, Y^{v-1}, Y}\left(y_{k} \mid x^{v-1}, y^{v-1}, y_{v}\right) \text { if } k=v+1 \\
p_{Y_{k-1} \mid X^{k-2}, Y^{k-2}}\left(y_{k} \mid x_{[1: k-1] \backslash\{v\}}, y_{[1: k-1] \backslash\{v\}}\right) \text { if } k>v+1
\end{array}\right.
$$

and

$$
\sum_{x_{v}, y_{v}} p^{*}\left(x^{N+1}, y^{N+1}\right)=p^{*}\left(x^{v-1}, x_{v+1}^{N+1}, y^{v-1}, y_{v+1}^{N+1}\right)
$$

Then, if $p^{*}$ is achievable for the $(N+1)$-node ADMN, $p^{*}$ is achievable for the $N$-node ADMN.

Proof: The proof is straightforward from the observation that the $(N+1)$-node ADMN is obtained by introducing a virtual node, whose channel output is $Y$ and channel input is null, between nodes $v-1$ and $v$ in the $N$-node $\mathrm{ADMN}$ and reindexing the nodes.

Proposition 2: Consider an $N$-node ADMN

$$
\left(\mathcal{X}_{1}, \ldots, \mathcal{X}_{N}, \mathcal{Y}_{1}, \ldots, \mathcal{Y}_{N}, \prod_{k=1}^{N} p\left(y_{k} \mid y^{k-1}, x^{k-1}\right)\right)
$$

such that $p\left(y_{v_{1}} \mid y^{v_{1}-1}, x^{v_{1}-1}\right)=p\left(y_{v_{1}}\right)$ and $p\left(y_{v_{2}} \mid y^{v_{2}-1}, x^{v_{2}-1}\right)=p\left(y_{v_{2}} \mid y_{v_{1}}\right)$ for some $v_{1} \in[1: N]$, $v_{2} \in[1: N], v_{1}<v_{2}$. Let $Y$ denote the common part of two random variables $Y_{v_{1}}$ and $Y_{v_{2}}$, where the common part of two discrete memoryless sources is defined in [49], [50]

Now, consider an $(N+1)$-node ADMN

$$
\left(\mathcal{X}_{1}^{\prime}, \ldots, \mathcal{X}_{N+1}^{\prime}, \mathcal{Y}_{1}^{\prime}, \ldots, \mathcal{Y}_{N+1}^{\prime}, \prod_{k=1}^{N+1} p^{\prime}\left(y_{k} \mid y^{k-1}, x^{k-1}\right)\right)
$$

and target distribution $p^{*}$ such that

$$
\mathcal{X}_{k}^{\prime}=\left\{\begin{array}{l}
\mathcal{X}_{k} \text { if } k<v_{1} \\
\mathcal{X} \text { if } k=v_{1} \\
\mathcal{X}_{k-1} \text { if } k>v_{1}
\end{array} \quad, \quad \mathcal{Y}_{k}^{\prime}=\left\{\begin{array}{l}
\mathcal{Y}_{k} \text { if } k<v_{1} \\
\mathcal{Y} \text { if } k=v_{1} \\
\mathcal{Y}_{k-1} \times \mathcal{X} \text { if } k=v_{1}+1 \text { or } k=v_{2}+1 \\
\mathcal{Y}_{k-1} \text { otherwise }
\end{array},\right.\right.
$$




$$
p^{\prime}\left(y_{k} \mid x^{k-1}, y^{k-1}\right)=\left\{\begin{array}{l}
p_{Y_{k} \mid X^{k-1}, Y^{k-1}}\left(y_{k} \mid x^{k-1}, y^{k-1}\right) \text { if } k<v_{1} \\
p_{Y}\left(y_{k}\right) \text { if } k=v_{1} \\
p_{Y_{v_{1}}}\left(y_{k, 1}\right) \mathbb{1}_{y_{k, 2}=x_{v_{1}}} \text { if } k=v_{1}+1 \\
p_{Y_{v_{2}} \mid Y_{v_{1}}}\left(y_{k, 1} \mid y_{v_{1}+1,1}\right) \mathbb{1}_{y_{k, 2}=x_{v_{1}}} \text { if } k=v_{2}+1 \\
p_{Y_{k-1} \mid X^{k-2}, Y^{k-2}}\left(y_{k} \mid x_{[1: k-1] \backslash\left\{v_{1}\right\}}, y_{[1: k-1] \backslash\left\{v_{1}\right\}}\right) \text { otherwise }
\end{array}\right.
$$

and

$$
\sum_{x_{v_{1}}, y_{v_{1}}} p^{\prime *}\left(x^{N+1}, y^{N+1}\right)=p^{*}\left(x^{v_{1}-1}, x_{v_{1}+1}^{N+1}, y^{v_{1}-1}, y_{v_{1}+1}^{N+1}\right)
$$

where $y_{k}=\left(y_{k, 1}, y_{k, 2}\right)$ for $k=v_{1}+1$ or $k=v_{2}+1$ and $|\mathcal{X}|$ can be arbitrarily large.

Then, if $p^{*}$ is achievable for the $(N+1)$-node ADMN, $p^{*}$ is achievable for the $N$-node ADMN.

Proof: Note that in the $N$-node ADMN, both nodes $v_{1}$ and $v_{2}$ observe the common part $Y$ and hence can share any function of $Y^{n}$. Thus, we can introduce a virtual node whose channel output is $Y$ and channel input is $X$ and assume that $X^{n}$ is available at nodes $v_{1}$ and $v_{2}$.

\section{UNIFIED CODING THEOREM}

In this section, we propose a unified coding scheme and present the main theorem of this paper, followed by various examples that show how to utilize our results. Our scheme consists of the following ingredients: 1) superposition, 2) simultaneous nonunique decoding, 3) simultaneous compression, and 4) symbol-by-symbol mapping. These are tweaked and combined in a special way to enable unification of many previous approaches. Let us first briefly explain the proposed scheme and introduce related coding parameters. Detailed description of our scheme is given in the proof of Theorem 1 .

- Codebook generation: In our coding scheme, covering codebooks are used to compress information that each node observes and decodes. We generate $\nu$ covering codebooks $\mathcal{C}_{1}, \ldots, \mathcal{C}_{\nu}$. Let $\mathcal{U}_{j}$ for $j \in[1: \nu]$ denote the alphabet for the codeword symbol of $\mathcal{C}_{j}$. For indexing of codewords, we consider $\mu$ index sets $\mathcal{L}_{1}, \ldots, \mathcal{L}_{\mu}$, where $\mathcal{L}_{j}=\left[1: 2^{n r_{j}}\right]$ for some $r_{j} \geq 0$ for each $j \in[1: \mu]$. We denote by $\Gamma_{j} \subseteq[1: \mu]$ the set of indices of $\mathcal{L}$ 's associated with $\mathcal{C}_{j}$ in a way that each codeword in $\mathcal{C}_{j}$ is indexed by the vector $l_{\Gamma_{j}} \in \prod_{i \in \Gamma_{j}} \mathcal{L}_{i}$ and hence $\mathcal{C}_{j}$ consists of $2^{n \sum_{i \in \Gamma_{j}} r_{i}}$ codewords, i.e., $\mathcal{C}_{j}=\left\{u_{j}^{n}\left(l_{\Gamma_{j}}\right): l_{\Gamma_{j}} \in \prod_{i \in \Gamma_{j}} \mathcal{L}_{i}\right\}$. Each codebook is constructed allowing superposition coding. Let $A_{j} \subseteq[1: \nu], j \in[1: \nu]$ denote the set of the indices of $\mathcal{C}$ 's on which $\mathcal{C}_{j}$ is constructed by superposition. 


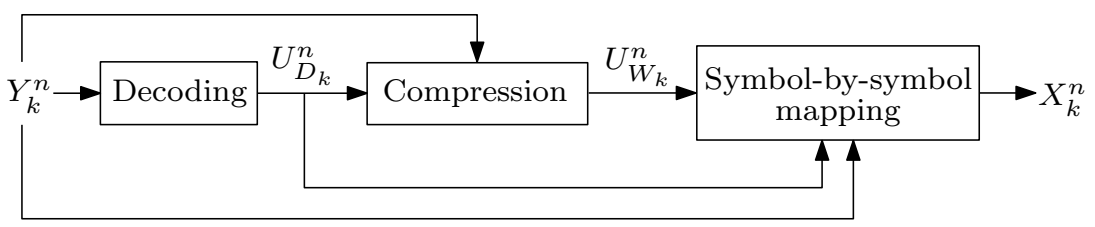

Fig. 5. Node $k \in[1: N]$ operates in three steps: 1) simultaneous nonunique decoding, 2) simultaneous compression, and 3) symbol-by-symbol mapping.

- Node operation: Node $k \in[1: N]$ operates according to the following three steps as illustrated in Fig. 5

- Simultaneous nonunique decoding: After receiving $y_{k}^{n}$, node $k$ decodes some covering codewords of previous nodes simultaneously, where some are decoded uniquely and the others are decoded non-uniquely. We denote by $D_{k} \subseteq[1: \nu]$ and $B_{k} \subseteq[1: \nu]$ the sets of the indices of $\mathcal{C}$ 's whose codewords are decoded uniquely and non-uniquely, respectively, at node $k$.

- Simultaneous compression: After decoding, node $k$ finds covering codewords $u_{W_{k}}^{n}$ simultaneously according to a conditional pmf $p\left(u_{W_{k}} \mid u_{D_{k}}, y_{k}\right)$ that carry some information about the received channel output sequence $y_{k}^{n}$ and uniquely decoded codewords $u_{D_{k}}^{n}$, where $W_{k} \subseteq[1: \nu]$ denotes the set of the indices of $\mathcal{C}$ 's used for compression.

- Symbol-by-symbol mapping: After decoding and compression, node $k$ generates $x_{k}^{n}$ by a symbolby-symbol mapping from uniquely decoded codewords $u_{D_{k}}^{n}$, covered codewords $u_{W_{k}}^{n}$, and received channel output sequence $y_{k}^{n}$. Let $x_{k}\left(u_{D_{k}}, u_{W_{k}}, y_{k}\right)$ denote the function used for symbolby-symbol mapping.

In summary, our scheme requires the following set $\omega$ of coding parameters, where some constraints are added to make the aforementioned codebook generation and node operation proper:

1) positive integers $\mu$ and $\nu$

2) alphabets $\mathcal{U}_{j}, j \in[1: \nu]$

3) $\mu$-rate tuple $\left(r_{1}, \ldots, r_{\mu}\right)$

4) sets $\Gamma_{j} \subseteq[1: \mu], A_{j} \subseteq[1: \nu], D_{k} \subseteq W^{k-1}, B_{k} \subseteq W^{k-1} \backslash D_{k}$, and $W_{k} \subseteq[1: \nu] \backslash W^{k-1}$ for $k \in[1: N]$ and $j \in[1: \nu]$ that satisfy

A-1 $\Gamma_{W_{k}} \backslash \Gamma_{D_{k}}$ 's are disjoint,

A-2 $\Gamma_{A_{j}} \subseteq \Gamma_{j}$ and $j^{\prime}<j$ if $j^{\prime} \in A_{j}$,

A-3 $A_{W_{k}} \subseteq W_{k} \cup D_{k}, A_{B_{k}} \subseteq D_{k} \cup B_{k}$, and $A_{D_{k}} \subseteq D_{k}$. 
5) a set of conditional pmfs $p\left(u_{W_{k}} \mid u_{D_{k}}, y_{k}\right)$ and functions $x_{k}\left(u_{D_{k}}, u_{W_{k}}, y_{k}\right)$ for $k \in[1: N]$ such that $p\left(x_{[1: N]}, y_{[1: N]}\right)$ induced by

$$
\prod_{k=1}^{N} p\left(y_{k} \mid y^{k-1}, x^{k-1}\right) p\left(u_{W_{k}} \mid u_{D_{k}}, y_{k}\right) \mathbb{1}_{x_{k}=x_{k}\left(u_{D_{k}}, u_{W_{k}}, y_{k}\right)}
$$

is the same as the target distribution $p^{*}\left(x_{[1: N]}, y_{[1: N]}\right)$.

Now, we are ready to present our main theorem, which gives a sufficient condition for achievability using the aforementioned scheme. For an $\operatorname{ADMN}\left(\mathcal{X}_{1}, \ldots, \mathcal{X}_{N}, \mathcal{Y}_{1}, \ldots, \mathcal{Y}_{N}, \prod_{k=1}^{N} p\left(y_{k} \mid y^{k-1}, x^{k-1}\right)\right)$ and target distribution $p^{*}$, let $\Omega\left(\mathcal{X}_{1}, \ldots, \mathcal{X}_{N}, \mathcal{Y}_{1}, \ldots, \mathcal{Y}_{N}, \prod_{k=1}^{N} p\left(y_{k} \mid y^{k-1}, x^{k-1}\right), p^{*}\right)$, shortly denoted as $\Omega\left(p^{*}\right)$ or $\Omega$, denote the set of all possible $\omega$ 's.

Theorem 1: For an $N$-node ADMN, $p^{*}$ is achievable if there exists $\omega \in \Omega$ such that for $1 \leq k \leq N$

$$
\begin{aligned}
& \sum_{j \in \bar{S}_{k}} r_{j}<\sum_{j \in S_{k}} I\left(U_{j} ; U_{S_{k}[j] \cup S_{k}^{c}}, Y_{k} \mid U_{A_{j}}\right) \\
& \sum_{j \in \bar{T}_{k}} r_{j}>\sum_{j \in T_{k}} I\left(U_{j} ; U_{T_{k}[j] \cup D_{k}}, Y_{k} \mid U_{A_{j}}\right)
\end{aligned}
$$

for all $\bar{S}_{k} \subseteq \bar{D}_{k} \cup \bar{B}_{k}$ such that $\bar{S}_{k} \cap \bar{D}_{k} \neq \emptyset$ and for all $\bar{T}_{k} \subseteq \bar{W}_{k}$ such that $\bar{T}_{k} \neq \emptyset$, where $\bar{D}_{k} \triangleq \Gamma_{D_{k}}$, $\bar{B}_{k} \triangleq \Gamma_{B_{k}} \backslash \Gamma_{D_{k}}, \bar{W}_{k} \triangleq \Gamma_{W_{k}} \backslash \Gamma_{D_{k}}$,

$$
\begin{aligned}
& S_{k} \triangleq\left\{j: j \in D_{k} \cup B_{k}, \Gamma_{j} \cap \bar{S}_{k} \neq \emptyset\right\}, \\
& T_{k} \triangleq\left\{j: j \in W_{k}, \Gamma_{j} \cap\left(\bar{T}_{k} \cup \bar{D}_{k}\right)^{c}=\emptyset\right\} .
\end{aligned}
$$

Remark 1: For $k \in[1: N]$, the inequalities (2) and (3) are the conditions for successful simultaneous nonunique decoding and simultaneous compression, respectively, at node $k$.

Remark 2: Theorem 1 can be improved using coded time sharing [19].

Proof: Consider $\omega \in \Omega$. Let $0<\epsilon_{k}<\epsilon_{k}^{\prime}<\epsilon_{k}^{\prime \prime}$ for all $k \in[1: N]$ such that $\epsilon_{k-1}^{\prime \prime}<\epsilon_{k}$ and $\epsilon_{N}^{\prime \prime}<\epsilon$. Let $\mathcal{L}_{j}=\left[1: 2^{n r_{j}}\right]$ for $j \in[1: \mu]$. In the following, $l_{j} \in \mathcal{L}_{j}$ for $j \in[1: \mu]$.

1) Codebook generation: For each $j \in[1: \nu]$ and $l_{\Gamma_{j}} \in \prod_{i \in \Gamma_{j}} \mathcal{L}_{i}$, generate $u_{j}^{n}\left(l_{\Gamma_{j}}\right)$ conditionally independently according to $\prod_{i=1}^{n} p\left(u_{j, i} \mid u_{A_{j}, i}\left(l_{\Gamma_{A_{j}}}\right)\right)$. Let $u_{S}^{n}\left(l_{\Gamma_{S}}\right)$ for $S \subseteq[1: \nu]$ denote $\left\{u_{i}^{n}\left(l_{\Gamma_{i}}\right): i \in S\right\}$.

2) Operation at node $k \in[1: N]$ : After receiving $Y_{k}^{n}$, node $k$ finds the smallest $\hat{l}_{\bar{D}_{k}, k}$ such that

$$
\left(u_{D_{k} \cup B_{k}}^{n}\left(\hat{l}_{\bar{D}_{k}, k}, l_{\bar{B}_{k}}\right), y_{k}^{n}\right) \in \mathcal{T}_{\epsilon_{k}}^{(n)}
$$

for some $l_{\bar{B}_{k}}$. If there is no such index vector, let $\hat{l}_{\bar{D}_{k}, k}=\mathbf{1}$. 
Next, node $k$ finds the smallest $l_{\bar{W}_{k}}$ such that

$$
\left(u_{D_{k}}^{n}\left(\hat{l}_{\bar{D}_{k}, k}\right), u_{W_{k}}^{n}\left(\hat{l}_{\bar{D}_{k}, k}, l_{\bar{W}_{k}}\right), y_{k}^{n}\right) \in \mathcal{T}_{\epsilon_{k}^{\prime}}^{(n)} .
$$

If there is no such index vector, let $l_{\bar{W}_{k}}=\mathbf{1}$. Send $x_{k, i}=x_{k}\left(u_{D_{k}, i}\left(\hat{l}_{\bar{D}_{k}, k}\right), u_{W_{k}, i}\left(\hat{l}_{\bar{D}_{k}, k}, l_{\bar{W}_{k}}\right), y_{k, i}\right)$ for $i \in[1: n]$.

3) Error analysis: For $k \in[1: N]$, let $\hat{L}_{\bar{D}_{k}, k}$ and $L_{\bar{W}_{k}}$ denote the chosen index vectors at node $k$. Let us define the error event as follows:

$$
\mathcal{E}=\bigcup_{k=1}^{N}\left(\mathcal{E}_{k, 1} \cup \mathcal{E}_{k, 2} \cup \mathcal{E}_{k, 3} \cup \mathcal{E}_{k, 4}\right)
$$

where

$$
\begin{aligned}
& \mathcal{E}_{k, 1}=\left\{\left(U_{W^{k-1}}^{n}\left(L_{\bar{W}^{k-1}}\right), Y_{[1: k]}^{n}\right) \notin \mathcal{T}_{\epsilon_{k}}^{(n)}\right\} \\
& \mathcal{E}_{k, 2}=\left\{\left(U_{D_{k} \cup B_{k}}^{n}\left(l_{\bar{D}_{k} \cup \bar{B}_{k}}\right), Y_{k}^{n}\right) \in \mathcal{T}_{\epsilon_{k}}^{(n)} \text { for some } l_{\bar{D}_{k}} \neq L_{\bar{D}_{k}}, l_{\bar{B}_{k}}\right\} \\
& \mathcal{E}_{k, 3}=\left\{\left(U_{D_{k}}^{n}\left(\hat{L}_{\bar{D}_{k}, k}\right), U_{W_{k}}^{n}\left(\hat{L}_{\bar{D}_{k}, k}, L_{\bar{W}_{k}}\right), Y_{k}^{n}\right) \notin \mathcal{T}_{\epsilon_{k}^{\prime}}^{(n)}\right\} \\
& \mathcal{E}_{k, 4}=\left\{\left(U_{W^{k}}^{n}\left(L_{\bar{W}^{k}}\right), Y_{[1: k]}^{n}\right) \notin \mathcal{T}_{\epsilon_{k}^{\prime \prime}}^{(n)}\right\} .
\end{aligned}
$$

Note that $\mathcal{E}^{c}$ implies $\hat{L}_{\bar{D}_{k}, k}=L_{\bar{D}_{k}}$ for all $k \in[1: N]$ and $\left(U_{W^{N}}^{n}\left(L_{\bar{W}^{N}}\right), Y_{[1: N]}^{n}\right) \in \mathcal{T}_{\epsilon}^{(n)}$, which means $\left(X_{[1: N]}^{n}, Y_{[1: N]}^{n}\right) \in \mathcal{T}_{\epsilon}^{(n)}$. Hence, $P_{e}^{(n)}(\epsilon) \leq P(\mathcal{E})$.

The probability of the error event can be upper-bounded as follows:

$$
\begin{gathered}
P(\mathcal{E}) \leq \sum_{k=1}^{N}\left(P\left(\mathcal{E}_{k, 1} \cap \bigcap_{j=1}^{k-1}\left(\mathcal{E}_{j, 1} \cup \mathcal{E}_{j, 2}\right)^{c} \cap \mathcal{E}_{k-1,4}^{c}\right)+P\left(\mathcal{E}_{k, 2} \cap \bigcap_{j=1}^{k-1}\left(\mathcal{E}_{j, 1} \cup \mathcal{E}_{j, 2} \cup \mathcal{E}_{j, 3}\right)^{c}\right)\right. \\
\left.\quad+P\left(\mathcal{E}_{k, 3} \cap \mathcal{E}_{k, 1}^{c} \cap \mathcal{E}_{k, 2}^{c}\right)+P\left(\mathcal{E}_{k, 4} \cap \mathcal{E}_{k, 1}^{c} \cap \mathcal{E}_{k, 2}^{c}\right)\right) .
\end{gathered}
$$

Note that $\left(\mathcal{E}_{k, 1} \cup \mathcal{E}_{k, 2}\right)^{c}$ implies $\hat{L}_{\bar{D}_{k}, k}=L_{\bar{D}_{k}}$.

Let us bound each term in the summation in (8) for given $k \in[1: N]$. First, we have

$$
\begin{aligned}
& P\left(\mathcal{E}_{k, 1} \cap \bigcap_{j=1}^{k-1}\left(\mathcal{E}_{j, 1} \cup \mathcal{E}_{j, 2}\right)^{c} \cap \mathcal{E}_{k-1,4}^{c}\right) \\
& \leq P\left(\left(U_{W^{k-1}}^{n}\left(L_{\bar{W}^{k-1}}\right), Y_{[1: k-1]}^{n}\right) \in \mathcal{T}_{\epsilon_{k-1}^{\prime \prime}}^{(n)},\left(U_{W^{k-1}}^{n}\left(L_{\bar{W}^{k-1}}\right), Y_{[1: k-1]}^{n}, Y_{k}^{n}\right) \notin \mathcal{T}_{\epsilon_{k}}^{(n)},\right. \\
& \left.\hat{L}_{\bar{D}_{j}, j}=L_{\bar{D}_{j}} \text { for all } j \in[1: k-1]\right),
\end{aligned}
$$

which tends to zero as $n$ tends to infinity from the conditional typicality lemma [41].

${ }^{2}$ In 77, $\left(\hat{l}_{\Gamma_{W_{k}} \backslash \bar{W}_{k}, k}, l_{\bar{W}_{k}}\right)$ suffices to specify the index set of $u_{W_{k}}^{n}$, but we write $\left(\hat{l}_{\bar{D}_{k}, k}, l_{\bar{W}_{k}}\right)$ as the index set of $u_{W_{k}}^{n}$ for notational convenience. 
Next, we show in Appendix $\mathrm{A}$ that the second term in the summation in (8) tends to zero as $n$ tends to infinity if

$$
\sum_{j \in \bar{S}_{k}} r_{j}<\sum_{j \in S_{k}} I\left(U_{j} ; U_{S_{k}[j] \cup S_{k}^{c}}, Y_{k} \mid U_{A_{j}}\right)-(1+\nu) \delta\left(\epsilon_{k}\right)
$$

for all $\bar{S}_{k} \subseteq \bar{D}_{k} \cup \bar{B}_{k}$ such that $\bar{S}_{k} \cap \bar{D}_{k} \neq \emptyset$, where $S_{k}$ is defined in (4).

The third term in the summation in (8) is shown in Appendix B to tend to zero as $n$ tends to infinity if

$$
\sum_{j \in \bar{T}_{k}} r_{j}>\sum_{j \in T_{k}} I\left(U_{j} ; U_{T_{k}[j] \cup D_{k}}, Y_{k} \mid U_{A_{j}}\right)+4(1+\nu) \delta\left(\epsilon_{k}^{\prime}\right)
$$

for all $\bar{T}_{k} \subseteq \bar{W}_{k}$ such that $\bar{T}_{k} \neq \emptyset$, where $T_{k}$ is defined in (5).

Finally, the fourth term in the summation in $(8)$ is proved in Appendix $\mathrm{C}$ to tend to zero as $n$ tends to infinity for sufficiently small $\epsilon_{k}$ and $\epsilon_{k}^{\prime}$ under the aforementioned condition (9) for all $\bar{T}_{k} \subseteq \bar{W}_{k}$ such that $\bar{T}_{k} \neq \emptyset$.

Therefore, $P(\mathcal{E})$ and thus $P_{e}^{(n)}(\epsilon)$ tend to zero as $n$ tends to infinity if rate tuple $\left(r_{1}, \ldots, r_{\mu}\right)$ satisfies for $1 \leq k \leq N$,

$$
\begin{aligned}
& \sum_{j \in \bar{S}_{k}} r_{j}<\sum_{j \in S_{k}} I\left(U_{j} ; U_{S_{k}[j] \cup S_{k}^{c}}, Y_{k} \mid U_{A_{j}}\right) \\
& \sum_{j \in \bar{T}_{k}} r_{j}>\sum_{j \in T_{k}} I\left(U_{j} ; U_{T_{k}[j] \cup D_{k}}, Y_{k} \mid U_{A_{j}}\right)
\end{aligned}
$$

for all $\bar{S}_{k} \subseteq \bar{D}_{k} \cup \bar{B}_{k}$ such that $\bar{S}_{k} \cap \bar{D}_{k} \neq \emptyset$ and for all $\bar{T}_{k} \subseteq \bar{W}_{k}$ such that $\bar{T}_{k} \neq \emptyset$. This completes the proof.

Let $\Omega^{\prime}$ denote the set of all possible $\omega$ 's that satisfy additional conditions $\nu=\mu$ and $\Gamma_{j}=\{j\} \cup A_{j}$. In many cases, it is sufficient to consider $\Omega^{\prime}$.

Corollary 1: For an $N$-node ADMN, $p^{*}$ is achievable if there exists $\omega^{\prime} \in \Omega^{\prime}$ such that for $1 \leq k \leq N$

$$
\begin{gathered}
\sum_{j \in S_{k}} r_{j}<\sum_{j \in S_{k}} I\left(U_{j} ; U_{S_{k}[j] \cup S_{k}^{c}}, Y_{k} \mid U_{A_{j}}\right) \\
\sum_{j \in T_{k}} r_{j}>\sum_{j \in T_{k}} I\left(U_{j} ; U_{T_{k}[j] \cup D_{k}}, Y_{k} \mid U_{A_{j}}\right)
\end{gathered}
$$

for all $S_{k} \subseteq D_{k} \cup B_{k}$ such that $S_{k} \cap D_{k} \neq \emptyset$ and if $j \in S_{k}^{c}$, then $A_{j} \subseteq S_{k}^{c}$ and for all $T_{k} \subseteq W_{k}$ such that $T_{k} \neq \emptyset$ and if $j \in T_{k}$, then $A_{j} \cap W_{k} \subseteq T_{k}$.

Proof: For $\omega^{\prime} \in \Omega^{\prime}$, we have $\bar{W}_{k}=W_{k}, \bar{D}_{k}=D_{k}, \bar{B}_{k}=B_{k}, \bar{S}_{k} \subseteq S_{k}$, and $T_{k} \subseteq \bar{T}_{k}$ for all $\bar{S}_{k} \subseteq \bar{D}_{k} \cup \bar{B}_{k}$ such that $\bar{S}_{k} \cap \bar{D}_{k} \neq \emptyset$ and for all $\bar{T}_{k} \subseteq \bar{W}_{k}$ such that $\bar{T}_{k} \neq \emptyset$. Hence, it is enough to consider $\bar{S}_{k}$ and $\bar{T}_{k}$ such that $\bar{S}_{k}=S_{k}$ and $\bar{T}_{k}=T_{k}$. 
Now, let us show various examples to illustrate how to utilize the unified framework and unified coding theorem. Throughout this paper, unspecified components $W_{k}, D_{k}, B_{k}$, and $A_{j}$ of $\omega \in \Omega$ or $\omega^{\prime} \in \Omega^{\prime}$ are assumed to be empty 3 .

\section{A. Multicoding and binning}

Our scheme does not include multicoding and binning explicitly, but our scheme is general enough to emulate those. In the following two examples, we provide guidelines for choosing $\omega$ that correpond multicoding and binning operations.

Example 6 (Gelfand-Pinsker coding [17]): Consider channels with noncausal states represented by a two-node ADMN such that $Y_{1}=(M, S), p\left(y_{1}\right)=p(m) p(s)$ where $H(M)=R$, and $p\left(y_{2} \mid y_{1}, x_{1}\right)=$ $p\left(y_{2} \mid x_{1}, s\right)$, as discussed in Section 【. We choose $\omega^{\prime} \in \Omega^{\prime}$ in Corollary 1 as $\mu=1, U_{1}=(M, U), W_{1}=$ $\{1\}, D_{2}=\{1\}, p\left(u \mid y_{1}\right)=p(u \mid s), x_{1}\left(u_{1}, y_{1}\right)=x_{1}(u, s)$, and $x_{2}\left(u_{1}, y_{2}\right)=m$. Then, from Corollary 1 we have the condition $r_{1}>R+I(U ; S)$ for compression at node 1 and the condition $r_{1}<I\left(U ; Y_{2}\right)$ for decoding at node 2. By the Fourier-Motzkin (F-M) elimination, we obtain $R<I\left(U ; Y_{2}\right)-I(U ; S)$.

To see the relevance to the multicoding operation, let us assume that $2^{R}$ is an integer and $M \sim \operatorname{Unif}[1$ : $\left.2^{R}\right]$. In this case, there are roughly $2^{n R}$ sets of $u_{1}^{n}$ sequences where each set consists of roughly $2^{n\left(r_{1}-R\right)}$ $u_{1}^{n}$ sequences having the same $m^{n}$ sequence. Note that $r_{1}-R>I(U ; S)$. Then, when $y_{1}^{n}=\left(\tilde{m}^{n}, \tilde{s}^{n}\right)$ is received at node 1 , among $2^{n\left(r_{1}-R\right)} u_{1}^{n}$ 's having $\tilde{m}^{n}$, we can find a $\tilde{u}_{1}^{n}=\left(\tilde{u}^{n}, \tilde{m}^{n}\right)$ with high probability such that $\tilde{u}^{n}$ is jointly typical with $\tilde{s}^{n}$ with respect to $p(u, s)$. This corresponds to the multicoding operation in a sense that for each message $m^{n}$, multiple $u^{n}$ 's are matched to satisfy the joint typicality with respect to $p(u, s)$.

Example 7 (Wyner-Ziv coding [26]): Consider lossy source coding with side information represented by a two-node ADMN such that $Y_{2}=\left(Y_{2}^{\prime}, T\right)$ and $p\left(y_{2} \mid y_{1}, x_{1}\right)=p\left(y_{2}^{\prime} \mid x_{1}\right) p\left(t \mid y_{1}\right)$ where $\max _{p\left(x_{1}\right)} I\left(X_{1} ; Y_{2}^{\prime}\right)$ $=R$, and target distribution $p^{*}$ such that $p^{*}\left(x_{1}\right)=\arg \max I\left(X_{1} ; Y_{2}^{\prime}\right)$ and $\mathrm{E}\left[d\left(Y_{1}, X_{2}\right)\right] \leq \frac{d}{1+\epsilon}$ for $\epsilon \rightarrow 0$, as discussed in Section $\amalg$. We choose $\omega^{\prime} \in \Omega^{\prime}$ in Corollary 1 as $\mu=1, W_{1}=\{1\}, D_{2}=\{1\}$, $U_{1}=\left(U, X_{1}\right), p\left(u_{1} \mid y_{1}\right)=p\left(u \mid y_{1}\right) p\left(x_{1}\right)$, and $x_{2}\left(u_{1}, y_{2}\right)=x_{2}(u, t)$ such that $p=p^{*}$. Then, from Corollary 1, we need the condition $r_{1}>I\left(U ; Y_{1}\right)$ for compression at node 1 and the condition $r_{1}<R+I(U ; T)$ for decoding at node 2. By the F-M elimination, we get $R>I\left(U ; Y_{1}\right)-I(U ; T)=I\left(U ; Y_{1} \mid T\right)$.

To see the relevance to the binning operation, let us assume that $2^{R}$ is an integer, $\left|\mathcal{X}_{1}\right|=2^{R}$, and $Y_{2}^{\prime}=X_{1}$. In this case, there are roughly $2^{n R}$ sets of $u_{1}^{n}$ sequences where each set consists of roughly

\footnotetext{
${ }^{3}$ For $\omega^{\prime} \in \Omega^{\prime}$, we do not explicitly specify $\Gamma_{j}$ since $\Gamma_{j}=\{j\} \cup A_{j}$.
} 
$2^{n\left(r_{1}-R\right)} u_{1}^{n}$ sequences having the same $x_{1}^{n}$ sequence. Note that $r_{1}-R<I(U ; T)$. Then, when $y_{2}^{n}=$ $\left(\tilde{x}_{1}^{n}, \tilde{t}^{n}\right)$ is received at node 2 , among $2^{n\left(r_{1}-R\right)} u_{1}^{n}$ sequences having $\tilde{x}_{1}^{n}$, there is a unique $\tilde{u}_{1}^{n}=\left(\tilde{u}^{n}, \tilde{x}_{1}^{n}\right)$ with high probability such that $\tilde{u}^{n}$ is jointly typical with $\tilde{t}^{n}$ with respect to $p(u, t)$. This corresponds to the binning operation in a sense that multiple $u^{n}$ sequences are matched to the same bin index $x_{1}^{n}$ but node 2 can decode $u^{n}$ using the joint typicality with respect to $p(u, t)$.

\section{B. Rate-splitting}

The following example shows how the rate-splitting can be incorporated.

Example 8 (Han-Kobayashi coding [19]): To perform the rate-splitting, we represent the interference channel as a four-node ADMN such that $Y_{k}=\left(M_{k 0}, M_{k k}\right), H\left(M_{k 0}\right)=R_{k 0}, H\left(M_{k k}\right)=R_{k k}, R_{k}=$ $R_{k 0}+R_{k k}$ for $k \in[1: 2], p\left(y_{1}\right)=p\left(m_{10}\right) p\left(m_{11}\right), p\left(y_{2} \mid y_{1}, x_{1}\right)=p\left(m_{20}\right) p\left(m_{22}\right), p\left(y_{3} \mid y_{[1: 2]}, x_{[1: 2]}\right)=$ $p\left(y_{3} \mid x_{[1: 2]}\right)$, and $p\left(y_{4} \mid y_{[1: 3]}, x_{[1: 3]}\right)=p\left(y_{4} \mid y_{3}, x_{[1: 2]}\right)$, and target distribution $p^{*}$ such that $X_{3}=Y_{1}, X_{4}=$ $Y_{2}$. Here, $M_{k 0}^{n}$ and $M_{k k}^{n}$ for $k \in[1: 2]$ correspond to the rate-splitted messages at node $k$.

We choose $\omega^{\prime} \in \Omega^{\prime}$ in Corollary 1 as follows: $\mu=4, U_{1}=\left(M_{10}, V_{1}\right), U_{2}=\left(M_{11}, X_{1}\right), U_{3}=$ $\left(M_{20}, V_{2}\right), U_{4}=\left(M_{22}, X_{2}\right), W_{1}=\{1,2\}, W_{2}=\{3,4\}, D_{3}=\{1,2\}, D_{4}=\{3,4\}, B_{3}=\{3\}, B_{4}=$ $\{1\}, A_{2}=\{1\}, A_{4}=\{3\}, p\left(v_{k}, x_{k} \mid y_{k}\right)=p\left(v_{k}, x_{k}\right)$ for $k \in[1: 2]$. Then, from Corollary 1 followed by the F-M elimination, we get the Han-Kobayashi inner bound.

\section{Introduction of a virtual node}

Let us show an example where a simpler rate expression than previously known result can be obtained by using Proposition 1 .

Example 9 (Interference decoding for a 3-SD-pair deterministic interference channel [22]]): In the 3SD-pair deterministic channel [22], source $k \in[1: 3]$ encodes a message $I_{k}$ of rate $R_{k} \geq 0$ to channel input sequence $X_{k}^{n}$ and destination $k$ estimates $I_{k}$ as $\hat{I}_{k}$ from its channel output sequence $Z_{k}^{n}$, where $n$ denotes the number of channel uses. The channel output at destination $k \in[1: 3]$ is given as $Z_{k}=$ $f_{k}\left(X_{k, k}, V_{k}\right)$ for some function $f_{k}$, where $X_{i, j}=g_{i, j}\left(X_{i}\right)$ for some function $g_{i, j}$ for $i \in[1: 3]$ and $j \in[1: 3], V_{1}=h_{1}\left(X_{2,1}, X_{3,1}\right), V_{2}=h_{2}\left(X_{1,2}, X_{3,2}\right)$, and $V_{3}=h_{3}\left(X_{1,3}, X_{2,3}\right)$ for some functions $h_{1}$, $h_{2}$, and $h_{3} . h_{k}$ and $f_{k}$ for $k \in[1: 3]$ are assumed to be injective in their arguments. The probability or error and achievability of a rate triple $\left(R_{1}, R_{2}, R_{3}\right)$ are defined in the standard way. In Fig. 6 , the 3-SD-pair deterministic channel is illustrated for destination 1. By using a new technique of decoding the combined interference, Bandemer and El Gamal showed in [22] the following achievable rate region. 4

\footnotetext{
${ }^{4}$ For simplicity, we present the rate region without coded time sharing.
} 


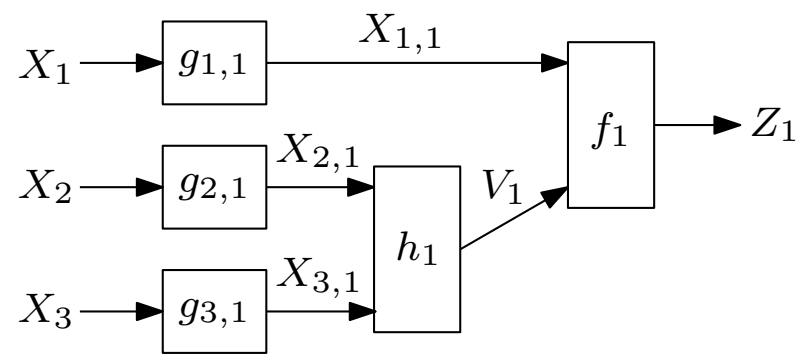

Fig. 6. The 3-SD-pair deterministic interference channel considered in [22]

Proposition 3 (Interference decoding inner bound [22]): The rate region $\bigcap_{k=1}^{3} \mathcal{R}_{k}\left(X_{1}, X_{2}, X_{3}\right)$ is achievable for some $\left(X_{1}, X_{2}, X_{3}\right) \sim p\left(x_{1}\right) p\left(x_{2}\right) p\left(x_{3}\right)$, where $\mathcal{R}_{1}\left(X_{1}, X_{2}, X_{3}\right)$ is the set of rate triples $\left(R_{1}, R_{2}, R_{3}\right)$ such that

$$
\begin{aligned}
R_{1} & <H\left(X_{1,1}\right) \\
R_{1}+\min \left(R_{2}, H\left(X_{2,1}\right)\right) & <H\left(Z_{1} \mid X_{3,1}\right) \\
R_{1}+\min \left(R_{3}, H\left(X_{3,1}\right)\right) & <H\left(Z_{1} \mid X_{2,1}\right) \\
R_{1}+\min \left(R_{2}+R_{3}, R_{2}+H\left(X_{3,1}\right), H\left(X_{2,1}\right)+R_{3}, H\left(V_{1}\right)\right) & <H\left(Z_{1}\right),
\end{aligned}
$$

and $\mathcal{R}_{2}\left(X_{1}, X_{2}, X_{3}\right)$ and $\mathcal{R}_{3}\left(X_{1}, X_{2}, X_{3}\right)$ are defined similarly by replacing the subscripts as $1 \mapsto 2 \mapsto$ $3 \mapsto 1$ and $1 \mapsto 3 \mapsto 2 \mapsto 1$ in $\mathcal{R}_{1}\left(X_{1}, X_{2}, X_{3}\right)$, respectively.

Now, let us show that Corollary 1 can recover the interference decoding inner bound by applying Proposition 1. By introducing a virtual node from Proposition 1, the 3-SD-pair deterministic channel can be represented by the following ADMN and target distribution:

- ADMN: $N=7, H\left(Y_{k}\right)=R_{k}$ for $k \in[1: 3], p\left(y_{2} \mid x_{1}, y_{1}\right)=p\left(y_{2}\right), p\left(y_{3} \mid x_{[1: 2]}, y_{[1: 2]}\right)=p\left(y_{3}\right)$, $Y_{4}=\left(X_{1,2}, X_{1,3}, X_{2,1}, X_{2,3}, X_{3,1}, X_{3,2}, V_{1}, V_{2}, V_{3}\right), X_{4}=\emptyset, Y_{5}=Z_{1}, Y_{6}=Z_{2}, Y_{7}=Z_{3}$.

- Target distribution: $p^{*}$ such that $X_{5}=Y_{1}, X_{6}=Y_{2}, X_{7}=Y_{3}$.

For this $\mathrm{ADMN}$ and $p^{*}$, let us choose $\omega^{\prime} \in \Omega^{\prime}$ for Corollary 1 We let $\mu=12, W_{k}=\{k\}$ for $k \in[1: 3], W_{4}=[4: 12], U_{j}=\left(Y_{j}, X_{j}\right)$ and $p\left(x_{j} \mid y_{j}\right)=p\left(x_{j}\right)$ for $j \in[1: 3], U_{4}=X_{1,2}, U_{5}=$ $X_{1,3}, U_{6}=X_{2,1}, U_{7}=X_{2,3}, U_{8}=X_{3,1}, U_{9}=X_{3,2}, U_{10}=V_{1}, U_{11}=V_{2}, U_{12}=V_{3}, D_{5}=\{1\}$, 
$D_{6}=\{2\}, D_{7}=\{3\}$. We let $B_{5}$ as follows:

$$
B_{5}=\left\{\begin{array}{ll}
\{10\} & \text { if } m_{1}=H\left(V_{1}\right) \\
\{2,3\} & \text { if } R_{2}<H\left(X_{2,1}\right), R_{3}<H\left(X_{3,1}\right), m_{1}<H\left(V_{1}\right) \\
\{2,8\} & \text { if } R_{2}<H\left(X_{2,1}\right), R_{3} \geq H\left(X_{3,1}\right), m_{1}<H\left(V_{1}\right) \\
\{3,6\} & \text { if } R_{2} \geq H\left(X_{2,1}\right), R_{3}<H\left(X_{3,1}\right), m_{1}<H\left(V_{1}\right)
\end{array},\right.
$$

where $m_{1}=\min \left(R_{2}+R_{3}, R_{2}+H\left(X_{3,1}\right), H\left(X_{2,1}\right)+R_{3}, H\left(V_{1}\right)\right)$. We choose $B_{6}$ and $B_{7}$ similarly. Then, by applying Corollary 1 we can obtain an inner bound that is at least as good as that in Proposition 3 and has a simpler form 5 . Furthermore, the interference decoding inner bound in [22] was improved in [23] by incorporating rate splitting, Marton coding, and superposition coding. We can choose $\omega^{\prime} \in \Omega^{\prime}$ that includes such coding techniques and obtain an inner bound from Corollary 1 that includes that in [23] and has a simpler form.

The following example illustrates the usefulness of Proposition 2 in networks with correlated sources.

Example 10 (Lossless communication of two correlated sources over a multiple access channel [30]): By using Proposition 2, we can represent the problem of sending two correlated sources over a multiple access channel as the following ADMN and target distribution:

- ADMN: $N=4, Y_{1}=V_{1}, Y_{2}=\left(V_{2}, X_{1}\right), Y_{3}=\left(V_{3}, X_{1}\right)$, and $p\left(y_{4} \mid y_{[1: 3]}, x_{[1: 3]}\right)=p\left(y_{4} \mid x_{[2: 3]}\right)$ where $V_{2}$ and $V_{3}$ are two discrete memoryless sources, $V_{1}$ is the common part of $V_{2}$ and $V_{3}$, and $\left|\mathcal{X}_{1}\right|$ is arbitrarily large.

- Target distribution: $p^{*}$ such that $X_{4}=\left(V_{2}, V_{3}\right)$.

We choose $\omega^{\prime} \in \Omega^{\prime}$ in Corollary 1 as follows: $\mu=3, U_{1}=\left(V_{1}, U, X_{1}\right), U_{2}=\left(V_{2}, X_{2}\right), U_{3}=\left(V_{3}, X_{3}\right)$, $W_{k}=\{k\}$ for $k \in[1: 3], D_{2}=\{1\}, D_{3}=\{1\}, D_{4}=\{1,2,3\}, A_{2}=\{1\}, A_{3}=\{1\}, p\left(u, x_{1} \mid y_{1}\right)=$ $p(u) /\left|\mathcal{X}_{1}\right|, p\left(x_{2} \mid y_{2}, u_{1}\right)=p\left(x_{2} \mid v_{2}, u\right), p\left(x_{3} \mid y_{3}, u_{1}\right)=p\left(x_{3} \mid v_{3}, u\right)$. By Corollary 1 followed by the F-M elimination, the sufficient condition for lossless communication of two correlated sources over a multiple access channel [30] is recovered.

\section{Application to DMNs}

Note that any strictly causal or causal network with blockwise operations can be represented as an ADMN by unfolding the network as illustrated in Section [I] The following lemma is useful when we

\footnotetext{
${ }^{5}$ When $m_{1}=H\left(V_{1}\right)$, our bound for the decoding at the first destination is given as $R_{1}<H\left(X_{1,1}\right)$ and $R_{1}+H\left(V_{1}\right)<H\left(Z_{1}\right)$, while that in Proposition 3 has two additional inequalities 111) and 12.
} 
apply Theorem 1 to unfolded networks.

Lemma 1: Consider $\omega \in \Omega$. For $\bar{S}_{k} \subseteq \bar{D}_{k} \cup \bar{B}_{k}$ such that $\bar{S}_{k} \cap \bar{D}_{k} \neq \emptyset$ and $\bar{T}_{k} \subseteq \bar{W}_{k}$ such that $\bar{T}_{k} \neq \emptyset$, the decoding and compression bounds, i.e., (2) and (3), in Theorem 1 are satisfied if

$$
\begin{aligned}
& \sum_{j \in \bar{S}_{k}} r_{j}<\sum_{j \in S_{k}^{\prime}} I\left(U_{j} ; U_{S_{k}^{\prime}}[j] \cup S_{k}^{\prime c}, Y_{k} \mid U_{A_{j}}\right) \\
& \sum_{j \in \bar{T}_{k}} r_{j}>\sum_{j \in T_{k}^{\prime}} I\left(U_{j} ; U_{T_{k}^{\prime}[j] \cup D_{k}}, Y_{k} \mid U_{A_{j}}\right),
\end{aligned}
$$

for some $S_{k}^{\prime} \subseteq S_{k}$ such that $A_{j} \subseteq\left(S_{k} \backslash S_{k}^{\prime}\right)[j] \cup S_{k}^{c}$ for all $j \in S_{k} \backslash S_{k}^{\prime}$ and for some $T_{k}^{\prime}$ such that $T_{k} \subseteq T_{k}^{\prime}$

Proof: The compression part is straightforward. For the decoding part, we have

$$
\begin{aligned}
& \sum_{j \in S_{k}^{\prime}} I\left(U_{j} ; U_{S_{k}^{\prime}[j] \cup S_{k}^{\prime c}}, Y_{k} \mid U_{A_{j}}\right) \\
& \stackrel{(a)}{\leq} \sum_{j \in S_{k}^{\prime}} I\left(U_{j} ; U_{S_{k}^{\prime}[j] \cup S_{k}^{\prime c}}, Y_{k} \mid U_{A_{j}}\right)+\sum_{j \in S_{k} \backslash S_{k}^{\prime}}\left(H\left(U_{j} \mid U_{A_{j}}\right)-H\left(U_{j} \mid U_{\left(S_{k} \backslash S_{k}^{\prime}\right)[j]}, U_{S_{k}^{c}}, Y_{k}\right)\right) \\
& =\sum_{j \in S_{k}} H\left(U_{j} \mid U_{A_{j}}\right)-H\left(U_{S_{k}^{\prime}} \mid U_{S_{k}^{\prime c}}, Y_{k}\right)-H\left(U_{S_{k} \backslash S_{k}^{\prime}} \mid U_{S_{k}^{c}}, Y_{k}\right) \\
& =\sum_{j \in S_{k}} H\left(U_{j} \mid U_{A_{j}}\right)-H\left(U_{S_{k}} \mid U_{S_{k}^{c}}, Y_{k}\right) \\
& =\sum_{j \in S_{k}}\left(H\left(U_{j} \mid U_{A_{j}}\right)-H\left(U_{j} \mid U_{S_{k}[j]}, U_{S_{k}^{c}}, Y_{k}\right)\right) \\
& =\sum_{j \in S_{k}} I\left(U_{j} ; U_{S_{k}[j] \cup S_{k}^{c}}, Y_{k} \mid U_{A_{j}}\right),
\end{aligned}
$$

where $(a)$ is from the condition for $S_{k}^{\prime}$ stated in the lemma.

In the following example, we show an achievable rate for a DMN by applying Theorem 1 to the unfolded network.

Example 11 (Noisy network coding [9]): Consider a single-source multicast DMN $\left(\mathcal{X}_{1}, \ldots, \mathcal{X}_{N}, \mathcal{Y}_{1}\right.$, $\left.\ldots, \mathcal{Y}_{N}, p\left(y_{[1: N]} \mid x_{[1: N]}\right)\right)$. Let node 1 denote the source node and let $\mathcal{D} \subseteq[2: N]$ denote the set of destination nodes. An $(R, n)$ code for the single-source multicast DMN consists of message $I$, uniformly distributed over $\mathcal{I} \triangleq\left[1: 2^{n R}\right]$, encoding function at the source that maps $I \in \mathcal{I}$ to $x_{1}^{n} \in \mathcal{X}_{1}^{n}$, processing function at node $k \in[2: N]$ at time $i \in[1: n]$ that maps $y_{k}^{i-1} \in \mathcal{Y}_{k}^{i-1}$ to $x_{k, i} \in \mathcal{X}_{k}$, and decoding function at destination $d \in \mathcal{D}$ that maps $y_{d}^{n} \in \mathcal{Y}_{d}^{n}$ to $\hat{I}_{d} \in \mathcal{I}$. The probability of error is defined as $P_{e}^{(n)}=P\left(\hat{I}_{d} \neq I\right.$ for some $\left.d \in \mathcal{D}\right)$ and a rate $R$ is said to be achievable if there exists a sequence of $(R, n)$ codes such that $\lim _{n \rightarrow \infty} P_{e}^{(n)}=0$. 
For a single-source multicast DMN, noisy network coding (NNC) rate [9] is given as follows.

Proposition 4 (Noisy network coding bound [9]): For a single-source multicast DMN, a rate of $R$ is achievable if

$$
R<\min _{d \in \mathcal{D}} \min _{S \in[2: N] \backslash\{d\}} I\left(X_{1}, X_{S} ; \hat{Y}_{S^{c}}, Y_{d} \mid X_{S^{c}}\right)-I\left(Y_{S} ; \hat{Y}_{S} \mid X^{N}, \hat{Y}_{S^{c}}, Y_{d}\right)
$$

for some $p_{X_{1}} \prod_{k=2}^{N} p_{X_{k}} p_{\hat{Y}_{k} \mid X_{k}, Y_{k}}$.

Now, let us obtain the NNC rate from Theorem 1. Fix $p_{X_{1}} \prod_{k=2}^{N} p_{X_{k}} p_{\hat{Y}_{k} \mid X_{k}, Y_{k}}$. Achievability uses $B$ transmission blocks, each consisting of $n$ channel uses. Let $Y_{k, b}^{n}$ and $X_{k, b}^{n}$ for $k \in[1: N]$ and $b \in[1: B]$ denote the channel output and channel input sequences, respectively, at node $k$ at block $b$. Let us assume the following blockwise operation at each node: at the end of block $b-1$, where $b \in[1: B]$, node $k \in[1: N]$ encodes what to transmit in block $b$, i.e., $X_{k, b}^{n}$, using previously received channel outputs up to block $b-1$, i.e., $Y_{k,[1: b-1]}^{n}$. Then, we can unfold the network.

In the unfolded network, we have $(B+1) N$ nodes. The operation of node $(k, b), k \in[1: N], b \in[1: B]$ corresponds to that of node $k$ of the original network transmitting in block $b$ based on the received channel outputs up to block $b-1$ and the operation of node $(d, B+1), d \in \mathcal{D}$ corresponds to that of node $d$ of the original network that estimates the message based on the received channel outputs up to block $B$. Let $Y_{k, b}^{\mathrm{unf}}$ and $X_{k, b}^{\mathrm{unf}}$ denote the channel output and channel input at node $(k, b)$ of the unfolded network, respectively. A message generated at the source is regarded as the channel output at node $(1,1)$, i.e., $Y_{1,1}^{\text {unf }}=M$ such that $H(M)=B R$, and the message estimate at destination $d \in \mathcal{D}$ is regarded as the channel input at node $(d, B+1)$, i.e., $\mathcal{X}_{d, B+1}^{\mathrm{unf}}=\mathcal{M}$. To reflect the fact that node $(k, b+1)$ is originally the same node as node $(k, b)$, we assume that node $(k, b)$ has an orthogonal link of sufficiently large rate to node $(k, b+1)$. Hence, for $k \in[1: N]$ and $b \in[1: B]$, we let $Y_{k, b+1}^{\mathrm{unf}}=\left(X_{k, b}^{\mathrm{unf}}, Y_{k, b}\right)$ and let

$$
p\left(y_{k, b} \mid y_{[1: N],[1: b]}^{\mathrm{unf}_{[1: k-1], b+1}}, x_{[1: N],[1: b]}^{\mathrm{unf}}, x_{[1: k-1], b+1}^{\mathrm{unf}}\right)=p_{Y_{k} \mid Y_{[1: k-1]}, X_{[1: N]}}\left(y_{k, b} \mid y_{[1: k-1], b}, x_{[1: N], b}\right)
$$

where $x_{k, b}^{\text {unf }}=\left(x_{k, b}, z_{k, b}\right)$ for $x_{k, b} \in \mathcal{X}_{k}$ and $z_{k, b} \in \mathcal{Z}_{k, b}$ for some $\mathcal{Z}_{k, b}$ with arbitrarily large cardinality. We assume a target joint distribution $p^{*}\left(x_{[1: N],[1: B+1]}^{\mathrm{unf}}, y_{[1: N],[1: B+1]}^{\mathrm{unf}}\right)$ such that $X_{d, B+1}^{\mathrm{unf}}=Y_{1,1}^{\mathrm{unf}}$ for all $d \in \mathcal{D}$. Note that the achievability of $p^{*}$ for the unfolded network implies the achievability of rate $R$ for the original network.

Now, let us choose $\omega \in \Omega$ to obtain the NNC rate. Let $\mu=B N-B+1$ and $\nu=2 B N-2 B-N+2$. Consider a $\mu$-rate tuple $\left(r_{0}, r_{k, b}: k \in[2: N], b \in[0: B-1]\right)$. For notational convenience, let us index the codebook $\mathcal{C}$ by the auxiliary random variable used for its generation, i.e., if a codebook consists of $u^{n}(1), \ldots, u^{n}\left(2^{n r}\right)$ generated conditionally independently according to $\prod_{i=1}^{n} p\left(u_{i} \mid v_{i}\right)$ for some $r \geq 0$ 
and $p(u \mid v)$, we denote the codebook by $\mathcal{C}_{U}$. In addition, we index the index set $\mathcal{L}$ in the following way: $\mathcal{L}_{l_{0}}=\left[1: 2^{n r_{0}}\right]$ and $\mathcal{L}_{l_{k, b}}=\left[1: 2^{n r_{k, b}}\right]$ for $k \in[2: N]$ and $b \in[0: B-1]$. The remaining coding parameters associated with each node are given as follows:

- Node $(1,1)$ :

$$
W_{1,1}=\left\{U_{0}\right\}, \Gamma_{U_{0}}=\left\{l_{0}\right\}, U_{0}=\left(Y_{1,1}^{\mathrm{unf}}, X_{1,1}, \ldots, X_{1, B}\right), p\left(x_{1,1}, \ldots, x_{1, B} \mid y_{1,1}^{\mathrm{unf}}\right)=\prod_{b \in[1: B]} p_{X_{1}}\left(x_{1, b}\right)
$$

- Node $(k, 1), k \in[2: N]$ :

$$
W_{k, 1}=\left\{X_{k, 1}\right\}, \Gamma_{X_{k, 1}}=\left\{l_{k, 0}\right\}, p\left(x_{k, 1} \mid y_{k, 1}^{\mathrm{unf}}\right)=p_{X_{k}}\left(x_{k, 1}\right)
$$

- Node $(1, b), b \in[2: B]$ :

$$
D_{1, b}=W_{1,1}
$$

- Node $(k, b), k \in[2: N], b \in[2: B]$ :

$$
\begin{aligned}
D_{k, b}=W_{k}^{b-1}, W_{k, b}=\left\{\hat{Y}_{k, b-1}, X_{k, b}\right\}, \Gamma_{\hat{Y}_{k, b-1}} & =\left\{l_{k, b-1}, l_{k, b-2}\right\}, \Gamma_{X_{k, b}}=\left\{l_{k, b-1}\right\}, A_{\hat{Y}_{k, b-1}}=\left\{X_{k, b-1}\right\} \\
p\left(W_{k, b} \mid D_{k, b}, y_{k, b}^{\mathrm{unf}}\right) & =p_{\hat{Y}_{k} \mid X_{k}, Y_{k}}\left(\hat{y}_{k, b-1} \mid x_{k, b-1}, y_{k, b-1}\right) p_{X_{k}}\left(x_{k, b}\right)
\end{aligned}
$$

- Node $(d, B+1), d \in \mathcal{D}$ :

$$
D_{d, B+1}=W_{d}^{B} \cup\left\{U_{0}\right\}, B_{d, B+1}=\left\{X_{k, b}, \hat{Y}_{k, b}, k \in[2: N] \backslash\{d\}, b \in[1: B-1]\right\}
$$

For $k \in[1: N]$ and $b \in[1: B]$, we let $X_{k, b}^{\mathrm{unf}}=\left(Y_{k,[1: b-1]}, W_{k, b}, D_{k, b}\right)$. For $d \in \mathcal{D}$, let $X_{d, B+1}^{\text {unf }}=U_{0}$. Note that the above choice of coding parameters shows the following blockwise i.i.d. property:

$$
\begin{aligned}
& p\left(x_{[1: N],[1: B-1]}, y_{[2: N],[1: B-1]}, \hat{y}_{[2: N],[1: B-1]}\right) \\
& =\prod_{b \in[1: B-1]}\left(p_{X_{1}}\left(x_{1, b}\right) \prod_{k \in[2: N]} p_{X_{k}}\left(x_{k, b}\right) p_{\hat{Y}_{k} \mid X_{k}, Y_{k}}\left(\hat{y}_{k, b} \mid x_{k, b}, y_{k, b}\right) p_{Y_{[2: N]} \mid X_{[1: N]}}\left(y_{[2: N], b} \mid x_{[1: N], b}\right)\right) .
\end{aligned}
$$

Now, we are ready to apply Theorem 1 to obtain the NNC rate. For each node in the unfolded network, the decoding and compression bounds i.e., (2) and (3), respectively, are given as follows.

- Compression at node $(1,1)$ : The bound for compression is given as follows:

$$
r_{0}>B R \text {. }
$$

- Compression at node $(k, 1), k \in[2: N]$ : It can be easily shown that the bound for compression is inactive.

- Decoding at node $(1, b), b \in[2: B]$ : Since $Y_{1, b}^{\text {unf }}=D_{1, b}$, the bound for decoding becomes inactive. 
- Decoding and compression at node $(k, b), k \in[2: N], b \in[2: B]$ : Since $Y_{k, b}^{\text {unf }}$ contains $D_{k, b}$, the bound for decoding becomes inactive. From the blockwise i.i.d. property (16), the bound for compression is given as

$$
r_{k, b-1}>I\left(\hat{Y}_{k} ; Y_{k} \mid X_{k}\right)
$$

- Decoding at node $(d, B+1), d \in \mathcal{D}$ : Since $D_{d, B+1}=W_{d}^{B} \cup\left\{U_{0}\right\}$ and $Y_{d, B+1}^{\text {unf }}$ contains $W_{d}^{B}$, we only need to consider $\bar{S}_{d, B+1} \subseteq\left\{l_{0}, l_{k, b}: k \in[2: N] \backslash\{d\}, b \in[0: B-1]\right\}$ such that $l_{0} \in \bar{S}_{d, B+1}$. Note that such $\bar{S}_{d, B+1}$ can be represented as $\left\{l_{0}\right\} \cup \bigcup_{b \in[0: B-1]}\left\{l_{k, b}: k \in S_{b}\right\}$ for some $S_{b} \subseteq[2: N] \backslash\{d\}$ for $b \in[0: B-1]$. Then, from Lemma 1 and using the blockwise i.i.d. property shown in (16), the bound for decoding is given as

$$
\begin{aligned}
& r_{0}+\sum_{b \in[0: B-1]} \sum_{k \in S_{b}} r_{k, b}<\sum_{b \in[1: B-1]}\left(I\left(X_{1} ; \hat{Y}_{S_{b-1}^{c}}, X_{S_{b-1}^{c}}, Y_{d}\right)\right. \\
& \left.+\sum_{k \in S_{b-1}} I\left(X_{k} ; X_{S_{b-1}[k]}, X_{1}, \hat{Y}_{S_{b-1}^{c}}, X_{S_{b-1}^{c}}, Y_{d}\right)+\sum_{k \in S_{b-1}} I\left(\hat{Y}_{k} ; \hat{Y}_{S_{b-1}[k]}, \hat{Y}_{S_{b-1}^{c}}, X^{N}, Y_{d} \mid X_{k}\right)\right) \\
& <\sum_{b \in[1: B-1]}\left(I\left(X_{1}, X_{S_{b-1}} ; \hat{Y}_{S_{b-1}^{c}}, Y_{d} \mid X_{S_{b-1}^{c}}\right)+\sum_{k \in S_{b-1}} I\left(\hat{Y}_{k} ; \hat{Y}_{S_{b-1}[k]}, \hat{Y}_{S_{b-1}^{c}}, X^{N}, Y_{d} \mid X_{k}\right)\right)
\end{aligned}
$$

for all $S_{b} \subseteq[2: N] \backslash\{d\}$ for $b \in[0: B-1]$.

Now, let $r_{k, b}=r_{k}, k \in[2: N], b \in[0: B-1]$ for some $r_{k} \geq 0$. Then, (18) is satisfied if

$$
r_{k}>I\left(\hat{Y}_{k} ; Y_{k} \mid X_{k}\right)
$$

for $k \in[2: N]$, and (19) is satisfied if

$$
\begin{aligned}
r_{0}<(B-1) \min _{S: S \subseteq[2: N] \backslash\{d\}}\left(I\left(X_{1}, X_{S} ; \hat{Y}_{S^{c}}, Y_{d} \mid X_{S^{c}}\right)+\sum_{k \in S} I\left(\hat{Y}_{k} ; \hat{Y}_{S[k]}, \hat{Y}_{S^{c}}, X^{N}, Y_{d} \mid X_{k}\right)-\sum_{k \in S} r_{k}\right) \\
-\sum_{k \in[2: N]} r_{k}
\end{aligned}
$$

for all $d \in \mathcal{D}$.

By performing F-M elimination to (17), (20), and (21) and by taking $B \rightarrow \infty$, the NNC rate in Proposition 4 is obtained.

\section{E. Wiretap channel}

We note that a secrecy constraint cannot be imposed by using our definition of achievability based on joint typicality. Nevertheless, we show that our unified coding scheme can be specialized to the scheme that achieves the secrecy capacity of the wiretap channel. 
Example 12 (Wiretap channel [51]): Consider a three-node ADMN such that $Y_{1}=\left(M, M_{1}, M_{2}\right)$, $H(M)=R, H\left(M_{1}\right)=R_{1}, H\left(M_{2}\right)=R_{2}, p\left(y_{1}\right)=p(m) p\left(m_{1}\right) p\left(m_{2}\right), p\left(y_{2} \mid y_{1}, x_{1}\right)=p\left(y_{2} \mid x_{1}\right)$, and $p\left(y_{3} \mid y_{[1: 2]}, x_{[1: 2]}\right)=p\left(y_{3} \mid y_{2}, x_{1}\right)$ and target distribution $p^{*}$ such that $X_{2}=M$. Here, nodes 1,2 , and 3 correspond to a source, legitimate destination, and wiretapper, respectively, in the wiretap channel. $M^{n}$ corresponds to the message and $M_{1}^{n}$ and $M_{2}^{n}$ play the roles of fictitious messages to confuse the wiretapper. We note that the achievability of $p^{*}$ implies the reliable communication to the legitimate destination, but does not guarantee the security.

If we choose $\omega^{\prime} \in \Omega^{\prime}$ in Corollary 1 as $\mu=2, U_{1}=\left(M, M_{1}, U\right), U_{2}=\left(M_{2}, X_{1}\right), W_{1}=\{1,2\}$, $D_{2}=\{1\}, A_{2}=\{1\}, p\left(u, x_{1} \mid y_{1}\right)=p\left(u, x_{1}\right)$, we obtain following set of inequalities from Corollary 1

- $T_{1}=\{1\}: r_{1}>R+R_{1}$

- $T_{1}=\{1,2\} r_{1}+r_{2}>R+R_{1}+R_{2}$

- $S_{1}=\{1\}: r_{1}<I\left(U ; Y_{2}\right)$

If $R_{1}>I\left(U ; Y_{3}\right)$ and $R_{2}>I\left(X_{1} ; Y_{3} \mid U\right)$ in addition to the above conditions, it can be shown through the analysis of the equivocation at the wiretapper that this coding scheme satisfies the secrecy constraint [51]. By the F-M elimination, the secrecy capacity $C_{S}=\max _{p\left(u, x_{1}\right)} I\left(U ; Y_{2}\right)-I\left(U ; Y_{3}\right)$ is recovered.

More examples recovered by the unified coding theorem are relegated to Appendix D

\section{DUALity}

In this section, we establish a duality theorem that shows interesting similarities among achievability conditions for ADMNs in dual relations. For an $N$-node ADMN

$$
\left(\mathcal{X}_{1}, \ldots, \mathcal{X}_{N}, \mathcal{Y}_{1}, \ldots, \mathcal{Y}_{N}, \prod_{k=1}^{N} p\left(y_{k} \mid y^{k-1}, x^{k-1}\right)\right)
$$

with target joint distribution $p^{*}\left(x_{[1: N]}, y_{[1: N]}\right)$, which we call the original problem in this section to distinguish from dual problems, we define three types of dual problems as follows:

- Type-I dual problem consists of an $N$-node $\operatorname{ADMN}\left(\mathcal{Y}_{1}, \ldots, \mathcal{Y}_{N}, \mathcal{X}_{1}, \ldots, \mathcal{X}_{N}, \prod_{k=1}^{N} p_{1}\left(x_{k} \mid y^{k-1}, x^{k-1}\right)\right)$, i.e., the input and output alphabets are swapped, and a target joint distribution $p_{1}^{*}\left(x_{[1: N]}, y_{[1: N]}\right)$.

- Type-II dual problem consists of an $N$-node $\operatorname{ADMN}\left(\mathcal{X}_{N}, \ldots, \mathcal{X}_{1}, \mathcal{Y}_{N}, \ldots, \mathcal{Y}_{1}, \prod_{k=1}^{N} p_{2}\left(y_{k} \mid y_{k+1}^{N}, x_{k+1}^{N}\right)\right)$, i.e., the order of nodes is reversed, and a target joint distribution $p_{2}^{*}\left(x_{[1: N]}, y_{[1: N]}\right)$.

- Type-III dual problem consists of an $N$-node $\operatorname{ADMN}\left(\mathcal{Y}_{N}, \ldots, \mathcal{Y}_{1}, \mathcal{X}_{N}, \ldots, \mathcal{X}_{1}, \prod_{k=1}^{N} p_{3}\left(x_{k} \mid y_{k+1}^{N}, x_{k+1}^{N}\right)\right)$, i.e., the input and output alphabets are swapped and the order of nodes is reversed, and a target joint distribution $p_{3}^{*}\left(x_{[1: N]}, y_{[1: N]}\right)$.

We note that $p_{t}^{*}\left(x_{[1: N]}, y_{[1: N]}\right)$ for $t \in[1: 3]$ is not necessarily the same as $p^{*}\left(x_{[1: N]}, y_{[1: N]}\right)$. 
For the coding parameters of unified coding for the original problem and its dual problems, we define $\Omega_{d}$ as the set of $\omega_{d}=\left(\mu, \mathcal{U}_{j}, r_{j}, W_{k}, D_{k}, p\left(u_{W_{k}} \mid u_{D_{k}}, y_{k}\right), p_{1}\left(u_{W_{k}} \mid u_{D_{k}}, x_{k}\right), p_{2}\left(u_{D_{k}} \mid u_{W_{k}}, y_{k}\right)\right.$, $p_{3}\left(u_{D_{k}} \mid u_{W_{k}}, x_{k}\right), x_{k}\left(u_{W_{k}}, u_{D_{k}}, y_{k}\right), y_{k, 1}\left(u_{W_{k}}, u_{D_{k}}, x_{k}\right), x_{k, 2}\left(u_{W_{k}}, u_{D_{k}}, y_{k}\right), y_{k, 3}\left(u_{W_{k}}, u_{D_{k}}, x_{k}\right)$ for $k \in$ $[1: N]$ and $j \in[1: \mu])$ such that

$$
\begin{array}{r}
\left(\mu, \mathcal{U}_{j}, r_{j}, W_{k}, D_{k}, B_{k}, A_{j}, p\left(u_{W_{k}} \mid u_{D_{k}}, y_{k}\right), x_{k}\left(u_{W_{k}}, u_{D_{k}}, y_{k}\right) \text { for } k \in[1: N], j \in[1: \mu]\right) \\
\in \Omega^{\prime}\left(\mathcal{X}_{1}, \ldots, \mathcal{X}_{N}, \mathcal{Y}_{1}, \ldots, \mathcal{Y}_{N}, \prod_{k=1}^{N} p\left(y_{k} \mid y^{k-1}, x^{k-1}\right), p^{*}\right) \\
\left(\mu, \mathcal{U}_{j}, r_{j}, W_{k}, D_{k}, B_{k}, A_{j}, p_{1}\left(u_{W_{k}} \mid u_{D_{k}}, x_{k}\right), y_{k, 1}\left(u_{W_{k}}, u_{D_{k}}, x_{k}\right) \text { for } k \in[1: N], j \in[1: \mu]\right) \\
\in \Omega^{\prime}\left(\mathcal{Y}_{1}, \ldots, \mathcal{Y}_{N}, \mathcal{X}_{1}, \ldots, \mathcal{X}_{N}, \prod_{k=1}^{N} p_{1}\left(x_{k} \mid y^{k-1}, x^{k-1}\right), p_{1}^{*}\right) \\
\left(\mu, \mathcal{U}_{j}, r_{j}, W_{k}, D_{k}, B_{k}, A_{j}, p_{2}\left(u_{D_{k}} \mid u_{W_{k}}, y_{k}\right), x_{k, 2}\left(u_{W_{k}}, u_{D_{k}}, y_{k}\right) \text { for } k \in[1: N], j \in[1: \mu]\right) \\
\in \Omega^{\prime}\left(\mathcal{X}_{N}, \ldots, \mathcal{X}_{1}, \mathcal{Y}_{N}, \ldots, \mathcal{Y}_{1}, \prod_{k=1}^{N} p_{2}\left(y_{k} \mid y_{k+1}^{N}, x_{k+1}^{N}\right), p_{2}^{*}\right) \\
\left(\mu, \mathcal{U}_{j}, r_{j}, W_{k}, D_{k}, B_{k}, A_{j}, p_{3}\left(u_{D_{k}} \mid u_{W_{k}}, x_{k}\right), y_{k, 3}\left(u_{W_{k}}, u_{D_{k}}, x_{k}\right) \text { for } k \in[1: N], j \in[1: \mu]\right) \\
\left.\in \Omega^{\prime}\left(\mathcal{Y}_{N}, \ldots, \mathcal{Y}_{1}, \mathcal{X}_{N}, \ldots, \mathcal{X}_{1}, \prod_{k=1}^{N} p_{3}\left(x_{k} \mid y_{k+1}^{N}, x_{k+1}^{N}\right)\right), p_{3}^{*}\right),
\end{array}
$$

where $B_{k}=A_{j}=\emptyset$ for $k \in[1: N], j \in[1: \mu]$. We note that for type I and type III dual problems, where the input and output alphabets are swapped, $y_{k, t}$ for $k \in[1: N]$ and $t \in\{1,3\}$ is the function used for the symbol-by-symbol maping from $\left(U_{W_{k}}^{n}, U_{D_{k}^{n}}, X_{k}^{n}\right)$ to the channel input $Y_{k}^{n}$. We also note that for type-II and type-III dual problems, where the node order is reversed, the roles of $W_{k}$ and $D_{k}$ are swapped, i.e., node $k$ decodes $U_{W_{k}}^{n}$ and compresses the channel output sequence and decoded codewords as $U_{D_{k}}^{n}$ for $k \in[1: N]$.

The following duality theorem is directly obtained from Corollary 1

Theorem 2: Consider $\omega_{d} \in \Omega_{d}$. For the original network, $p^{*}$ is achievable if for $1 \leq k \leq N$

$$
\begin{gathered}
\sum_{j \in S_{k}} r_{j}<\sum_{j \in S_{k}} I\left(U_{j} ; U_{S_{k}[j] \cup S_{k}^{c}}, Y_{k}\right) \\
\sum_{j \in T_{k}} r_{j}>\sum_{j \in T_{k}} I\left(U_{j} ; U_{T_{k}[j] \cup D_{k}}, Y_{k}\right)
\end{gathered}
$$

for all $S_{k} \subseteq D_{k}$ such that $S_{k} \neq \emptyset$ and for all $T_{k} \subseteq W_{k}$ such that $T_{k} \neq \emptyset$. 
For the type-I dual network, $p_{1}^{*}$ is achievable if for $1 \leq k \leq N$

$$
\begin{aligned}
\sum_{j \in S_{k}} r_{j} & <\sum_{j \in S_{k}} I_{1}\left(U_{j} ; U_{S_{k}[j] \cup S_{k}^{c}}, X_{k}\right) \\
\sum_{j \in T_{k}} r_{j} & >\sum_{j \in T_{k}} I_{1}\left(U_{j} ; U_{T_{k}[j] \cup D_{k}}, X_{k}\right)
\end{aligned}
$$

for all $S_{k} \subseteq D_{k}$ such that $S_{k} \neq \emptyset$ and for all $T_{k} \subseteq W_{k}$ such that $T_{k} \neq \emptyset$, where the mutual informations are evaluated using the distribution $\left.\prod_{k=1}^{N} p_{1}\left(u_{W_{k}} \mid u_{D_{k}}, x_{k}\right) \mathbb{1}_{y_{k}=y_{k, 1}\left(u_{W_{k}}, u_{D_{k}}, x_{k}\right.}\right) p_{1}\left(x_{k} \mid y^{k-1}, x^{k-1}\right)$.

For the type-II dual network, $p_{2}^{*}$ is achievable if for $1 \leq k \leq N$

$$
\begin{aligned}
& \sum_{j \in S_{k}} r_{j}<\sum_{j \in S_{k}} I_{2}\left(U_{j} ; U_{S_{k}[j] \cup S_{k}^{c}}, Y_{k}\right) \\
& \sum_{j \in T_{k}} r_{j}>\sum_{j \in T_{k}} I_{2}\left(U_{j} ; U_{T_{k}[j] \cup W_{k}}, Y_{k}\right)
\end{aligned}
$$

for all $S_{k} \subseteq W_{k}$ such that $S_{k} \neq \emptyset$ and for all $T_{k} \subseteq D_{k}$ such that $T_{k} \neq \emptyset$, where the mutual informations are evaluated using the distribution $\prod_{k=1}^{N} p_{2}\left(u_{D_{k}} \mid u_{W_{k}}, y_{k}\right) \mathbb{1}_{x_{k}=x_{k, 2}\left(u_{W_{k}}, u_{D_{k}}, y_{k}\right)} p_{2}\left(y_{k} \mid y_{k+1}^{N}, x_{k+1}^{N}\right)$.

For the type-III dual network, $p_{3}^{*}$ is achievable if for $1 \leq k \leq N$

$$
\begin{aligned}
\sum_{j \in S_{k}} r_{j} & <\sum_{j \in S_{k}} I_{3}\left(U_{j} ; U_{S_{k}[j] \cup S_{k}^{c}}, X_{k}\right) \\
\sum_{j \in T_{k}} r_{j} & >\sum_{j \in T_{k}} I_{3}\left(U_{j} ; U_{T_{k}[j] \cup W_{k}}, X_{k}\right)
\end{aligned}
$$

for all $S_{k} \subseteq W_{k}$ such that $S_{k} \neq \emptyset$ and for all $T_{k} \subseteq D_{k}$ such that $T_{k} \neq \emptyset$, where the mutual informations are evaluated using the distribution $\left.\prod_{k=1}^{N} p_{3}\left(u_{D_{k}} \mid u_{W_{k}}, x_{k}\right) \mathbb{1}_{y_{k}=y_{k, 3}\left(u_{W_{k}}, u_{D_{k}}, x_{k}\right.}\right) p_{3}\left(x_{k} \mid y_{k+1}^{N}, x_{k+1}^{N}\right)$.

Remark 3: Theorem 2 shows some similarities among achievability conditions for dual problems. In the achievability conditions (23) and (25) for type-I and type-III dual problems, respectively, $X_{k}$ and $Y_{k}$ are swapped from (22). In the achievability conditions (24) and (25) for type-II and type-III dual problems, respectively, $W_{k}$ and $D_{k}$ are swapped from (22).

Theorem 2 includes as special cases many known duality relationships. Examples include the duality between the point-to-point channel coding (original problem) and the point-to-point source coding (typeI and type-II dual problems) and the duality between Gelfand-Pinsker coding [17] (original problem) and Wyner-Ziv coding [26] (type-II dual problem). Furthermore, using Theorem 2, duality can be shown among the achievability results for multiple-access channel [2] (original problem), distributed source coding [27], [28] (type-I dual problem), multiple-description [48] (type-II dual problem), and broadcast channel [18] (type-III dual problem). Let us describe the last duality in detail. For the original network, consider the multiple-access channel in Example 1 represented as a three-node ADMN 
$\left(\mathcal{X}_{1}, \mathcal{X}_{2}, \mathcal{X}_{3}, \mathcal{Y}_{1}, \mathcal{Y}_{2}, \mathcal{Y}_{3}, \prod_{k=1}^{3} p\left(y_{k} \mid y^{k-1}, x^{k-1}\right)\right)$ such that $\mathcal{X}_{3}=\mathcal{X}_{3,1} \times \mathcal{X}_{3,2}, \mathcal{X}_{3,1}=\mathcal{Y}_{1}, \mathcal{X}_{3,2}=\mathcal{Y}_{2}$, $H\left(Y_{1}\right)=R_{1}, p\left(y_{2} \mid y_{1}, x_{1}\right)=p\left(y_{2}\right), H\left(Y_{2}\right)=R_{2}$, and $p\left(y_{3} \mid y_{[1: 2]}, x_{[1: 2]}\right)=p\left(y_{3} \mid x_{[1: 2]}\right)$ with a target distribution $p^{*}\left(x_{[1: 3]}, y_{[1: 3]}\right)$ such that $X_{3}=\left(Y_{1}, Y_{2}\right)$. For the type-I dual problem, in which the input and output alphabets are swapped, we consider the distributed source coding problem, by assuming $p_{1}\left(x_{1}\right)$, $p_{1}\left(x_{2} \mid x_{1}, y_{1}\right)=p_{1}\left(x_{2} \mid x_{1}\right)$, and $p_{1}\left(x_{3}\right)=p_{1}\left(x_{3,1} \mid y_{1}\right) p_{1}\left(x_{3,2} \mid y_{2}\right)$ such that $\max _{p\left(y_{1}\right)} I\left(Y_{1} ; X_{3,1}\right)=R_{1}$ and $\max _{p\left(y_{2}\right)} I\left(Y_{2} ; X_{3,2}\right)=R_{2}$, and assuming target distribution $p_{1}^{*}\left(x_{[1: 3]}, y_{[1: 3]}\right)$ similarly as in Example 2. Next, for the type-II dual problem, in which the order of nodes is reversed, we consider the multipledescription scenario without combined reconstruction, which is a special case of Example 4, by assuming $p_{2}\left(y_{3}\right), p_{2}\left(y_{2} \mid y_{3}, x_{3}\right)=p_{2}\left(y_{2} \mid x_{3,2}\right)$ such that $\max _{p\left(x_{3,2}\right)} I\left(X_{3,2} ; Y_{2}\right)=R_{2}$, and $p_{2}\left(y_{1} \mid y_{2}, y_{3}, x_{2}, x_{3}\right)=$ $p_{2}\left(y_{1} \mid x_{3,1}\right)$ such that $\max _{p\left(x_{3,1}\right)} I\left(X_{3,1} ; Y_{1}\right)=R_{1}$, and assuming target distribution $p_{2}^{*}\left(x_{[1: 3]}, y_{[1: 3]}\right)$ similarly as in Example 4. For the type-III dual problem, we consider the broadcast channel problem in Example 3 by assuming $p_{3}\left(x_{3}\right)=p_{3}\left(x_{3,1}\right) p_{3}\left(x_{3,2}\right)$ such that $H\left(X_{3,1}\right)=R_{1}$ and $H\left(X_{3,2}\right)=R_{2}$, $p_{3}\left(x_{2} \mid y_{3}, x_{3}\right)=p\left(x_{2} \mid y_{3}\right)$, and $p_{3}\left(x_{1} \mid y_{2}, y_{3}, x_{2}, x_{3}\right)=p_{3}\left(x_{1} \mid y_{3}, x_{2}\right)$, and assuming target distribution $p_{3}^{*}\left(x_{[1: 3]}, y_{[1: 3]}\right)$ such that $Y_{1}=X_{3,1}$ and $Y_{2}=X_{3,2}$.

Choose $\omega_{d} \in \Omega_{d}$ as follows: $\mu=2, \mathcal{U}_{1}=\mathcal{Y}_{1} \times \mathcal{X}_{1} \times \mathcal{V}_{1}, \mathcal{U}_{2}=\mathcal{Y}_{2} \times \mathcal{X}_{2} \times \mathcal{V}_{2}, W_{1}=\{1\}, W_{2}=\{2\}, D_{3}=$ $\{1,2\}$. For the multiple access channel problem, we have $U_{1}=\left(Y_{1}, X_{1}\right)$ and $U_{2}=\left(Y_{2}, X_{2}\right)$, where $p\left(x_{1} \mid y_{1}\right)=p\left(x_{1}\right)$ and $p\left(x_{2} \mid y_{2}\right)=p\left(x_{2}\right)$ such that $p=p^{*}$. For the distributed source coding problem, we let $U_{1}=\left(V_{1}, Y_{1}\right), U_{2}=\left(V_{2}, Y_{2}\right)$, and $y_{3,1}\left(u_{1}, u_{2}, x_{3}\right)=y_{3,1}\left(v_{1}, v_{2}\right)$, where $p_{1}\left(u_{1} \mid x_{1}\right)=p_{1}\left(y_{1}\right) p_{1}\left(v_{1} \mid x_{1}\right)$ and $p_{1}\left(u_{2} \mid x_{2}\right)=p_{1}\left(y_{2}\right) p_{1}\left(v_{2} \mid x_{2}\right)$ such that $p_{1}=p_{1}^{*}$. For the multiple description problem, we assume $U_{1}=\left(X_{1}, X_{3,1}\right)$ and $U_{2}=\left(X_{2}, X_{3,2}\right)$, where $p_{2}\left(u_{1}, u_{2} \mid y_{3}\right)=p_{2}\left(x_{3,1}\right) p_{2}\left(x_{3,2}\right) p_{2}\left(x_{1} \mid y_{3}\right) p_{2}\left(x_{2} \mid y_{3}\right)$ such that $p_{2}=p_{2}^{*}$. For the broadcast channel problem, we assume $U_{1}=\left(X_{3,1}, V_{1}\right), U_{2}=\left(X_{3,2}, V_{2}\right)$, and $y_{3,3}\left(u_{1}, u_{2}\right)=y_{3,3}\left(v_{1}, v_{2}\right)$ such that $p_{3}\left(v_{1}, v_{2} \mid x_{3}\right)=p_{3}\left(v_{1}, v_{2}\right)$ and $p_{3}=p_{3}^{*}$.

Now, we are ready to apply Theorem 2, For the multiple access channel, we obtain the following condition to achieve $p^{*}$ :

$$
\begin{aligned}
& r_{1}>I\left(U_{1} ; Y_{1}\right)=R_{1} \\
& r_{2}>I\left(U_{2} ; Y_{2}\right)=R_{2} \\
& r_{1}<I\left(U_{1} ; U_{2}, Y_{3}\right)=I\left(X_{1} ; Y_{3} \mid X_{2}\right) \\
& r_{2}<I\left(U_{2} ; U_{1}, Y_{3}\right)=I\left(X_{2} ; Y_{3} \mid X_{1}\right) \\
r_{1}+r_{2} & <I\left(U_{1}, U_{2} ; Y_{3}\right)+I\left(U_{1} ; U_{2}\right)=I\left(X_{1}, X_{2} ; Y_{3}\right),
\end{aligned}
$$


which corresponds to the capacity region of the multiple access channel [2] by performing the F-M elimination, taking the union over $p\left(x_{1}\right) p\left(x_{2}\right)$, and incorporating coded time sharing [19].

Next, for the distributed source coding problem, we obtain the following condition to achieve $p_{1}^{*}$ :

$$
\begin{aligned}
r_{1} & >I_{1}\left(U_{1} ; X_{1}\right)=I_{1}\left(V_{1} ; X_{1}\right) \\
r_{2} & >I_{1}\left(U_{2} ; X_{2}\right)=I_{1}\left(V_{2} ; X_{2}\right) \\
r_{1} & <I_{1}\left(U_{1} ; U_{2}, X_{3}\right)=R_{1}+I_{1}\left(V_{1} ; V_{2}\right) \\
r_{2} & <I_{1}\left(U_{2} ; U_{1}, X_{3}\right)=R_{2}+I_{1}\left(V_{1} ; V_{2}\right) \\
r_{1}+r_{2} & <I_{1}\left(U_{1}, U_{2} ; X_{3}\right)+I_{1}\left(U_{1} ; U_{2}\right)=R_{1}+R_{2}+I_{1}\left(V_{1} ; V_{2}\right),
\end{aligned}
$$

which corresponds to the Berger-Tung inner bound [27] by performing the F-M elimination.

Next, for the multiple description problem without combined reconstruction, we obtain the following condition to achieve $p_{2}^{*}$ :

$$
\begin{aligned}
& r_{1}<I_{2}\left(U_{1} ; Y_{1}\right)=R_{1} \\
& r_{2}<I_{2}\left(U_{2} ; Y_{2}\right)=R_{2} \\
& r_{1}>I_{2}\left(U_{1} ; Y_{3}\right)=I_{2}\left(X_{1} ; Y_{3}\right) \\
& r_{2}>I_{2}\left(U_{2} ; Y_{3}\right)=I_{2}\left(X_{2} ; Y_{3}\right) \\
r_{1}+ & r_{2}>I_{2}\left(U_{1}, U_{2} ; Y_{3}\right)+I_{2}\left(U_{1} ; U_{2}\right)=I_{2}\left(X_{1} ; Y_{3}\right)+I_{2}\left(X_{2} ; Y_{3}\right),
\end{aligned}
$$

which corresponds to the optimal rate-distortion region by performing the F-M elimination and taking the union over $p\left(x_{1} \mid y_{3}\right) p\left(x_{2} \mid y_{3}\right)$ that satisfies the distortion constraints.

Lastly, for the broadcast channel problem, we obtain the following condition to achieve $p_{3}^{*}$ :

$$
\begin{aligned}
r_{1} & <I_{3}\left(U_{1} ; X_{1}\right)=I_{3}\left(V_{1} ; X_{1}\right) \\
r_{2} & <I_{3}\left(U_{2} ; X_{2}\right)=I_{3}\left(V_{2} ; X_{2}\right) \\
r_{1} & >I_{3}\left(U_{1} ; X_{3}\right)=R_{1} \\
r_{2} & >I_{3}\left(U_{2} ; X_{3}\right)=R_{2} \\
r_{1}+r_{2} & >I_{3}\left(U_{1}, U_{2} ; X_{3}\right)+I_{3}\left(U_{1} ; U_{2}\right)=R_{1}+R_{2}+I_{3}\left(V_{1} ; V_{2}\right),
\end{aligned}
$$

which corresponds to the Marton bound [18] by performing the F-M elimination. 


\section{Generalized DeCode-Compress-AmPlify-AND-Forward}

In this section, as an application of our unified coding theorem, we present a generalized decodecompress-amplify-and-forward (GDCAF) bound for a single-source single-destination $N$-node ADMN, which includes hybrid coding [12] and distributed decode-and-forward (DDF) [13] bounds applied to layered networks as special cases. Furthermore, we show an example where our GDCAF scheme strictly outperforms many previously known schemes.

For a single-source single-destination $N$-node ADMN where node 1 and node $N$ are the source and the destination, respectively, we let $H\left(Y_{1}\right)=R$ for some $R \geq 0, p\left(y_{k} \mid x^{k-1}, y^{k-1}\right)=p\left(y_{k} \mid x^{k-1}, y_{2}^{k-1}\right)$ for $k \in[2: N]$, and $p^{*}$ such that $X_{N}=Y_{1}$, i.e., $Y_{1}^{n}$ corresponds to the message of rate $R$ that does not affect the remaining channels and node $N$ wishes to decode $Y_{1}^{n}$ reliably. The following theorem gives the GDCAF bound using Corollary 1

Theorem 3 (GDCAF bound for a single-source single-destination ADMN): For a single-source singledestination $N$-node ADMN, a rate of $R$ is achievable if

$$
\begin{gathered}
R<\min _{S, T: S \subseteq T \subseteq[2: N-1]} I\left(X_{1}, U_{S}, \hat{Y}_{T} ; \hat{Y}_{T^{c}}, Y_{N} \mid U_{S^{c}}\right)-\sum_{j \in T} I\left(\hat{Y}_{j} ; Y_{j} \mid U_{[2: N-1]}, \hat{Y}_{T[j]}, X_{1}\right) \\
+H\left(U_{S^{c}}\right)-\sum_{j \in S^{c}} H\left(U_{j} \mid Y_{j}\right)
\end{gathered}
$$

for some $p\left(x_{1}, u_{2}, \ldots, u_{N}\right) \prod_{j \in[2: N-1]} p\left(\hat{y}_{j} \mid y_{j}, u_{j}\right)$ and functions $x_{k}\left(u_{k}, \hat{y}_{k}, y_{k}\right)$ for $k \in[2: N-1]$ such that

$$
\sum_{j \in S^{\prime}} I\left(U_{j} ; U_{S^{\prime}[j]}\right)<\sum_{j \in S^{\prime}} I\left(U_{j} ; Y_{j}\right)
$$

for all $S^{\prime} \subseteq[2: N-1]$.

Remark 4: In the GDCAF bound, $U_{k}$ and $\hat{Y}_{k}$ for $k \in[2: N-1]$ correspond to the partial information about the message decoded by node $k$ and the compressed version of $Y_{k}$ at node $k$, respectively.

Remark 5: The GDCAF bound recovers hybrid coding bound [12] applied to layered networks by letting $U_{k}=\emptyset$ for $k \in[2: N-1]$.

Remark 6: The GDCAF bound recovers DDF bound [13] applied to layered networks by letting $\hat{Y}_{k}=\emptyset$ and $U_{k}=\left(V_{k}, X_{k}\right)$ for $k \in[2: N-1]$, and $p\left(x_{1}, u_{2}, \ldots, u_{N-1}\right)=\prod_{k=2}^{N-1} p\left(x_{k}\right) p\left(x_{1} \mid x_{2}^{N-1}\right) p\left(v_{2}^{N} \mid x^{N-1}\right)$.

Proof: We apply Corollary 1 to derive the GDCAF bound. We choose $\omega^{\prime} \in \Omega^{\prime}$ as follows: $\mu=2 N-2$, $W_{1}=[1: N], A_{N}=[1: N-1], p\left(u_{1}, \ldots, u_{N} \mid y_{1}\right)=\mathbb{1}_{u_{1}=y_{1}} \cdot p\left(u_{2}, \ldots, u_{N}\right), X_{1}=U_{N}, D_{k}=\{k\}$, $W_{k}=\{N+k-1\}, A_{N+k-1}=\{k\}$ for $k \in[2: N-1], D_{N}=\{1\}$, and $B_{N}=[2: 2 N-2]$. For notational convenience, let $r_{k}^{\prime}$ and $\hat{Y}_{k}$ denote $r_{N+k-1}$ and $U_{N+k-1}$, respectively, for $k \in[2: N-1]$. 


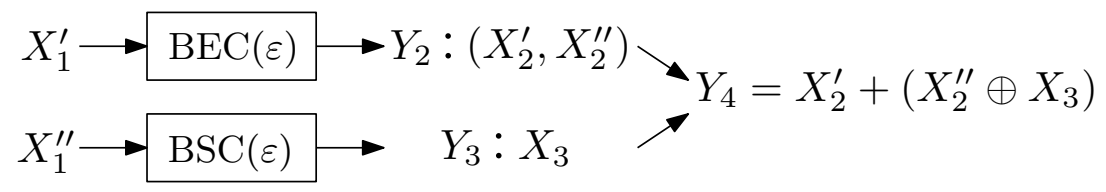

Fig. 7. An example of diamond network

By applying Corollary 1 for the aforementioned choice of $\omega^{\prime}$, we obtain the following bounds:

$$
\begin{aligned}
& r_{1}>R \\
& \sum_{j \in S} r_{j}>\sum_{j \in S} I\left(U_{j} ; U_{S[j]}\right) \\
& \sum_{j \in[1: N]} r_{j}>R+\sum_{j \in[2: N-1]} I\left(U_{j} ; U^{j-1}\right) \\
& r_{k}<I\left(U_{k} ; Y_{k}\right) \\
& r_{k}^{\prime}>I\left(\hat{Y}_{k} ; Y_{k} \mid U_{k}\right) \\
& r_{1}+r_{N}+\sum_{j \in S} r_{j}+\sum_{j \in T} r_{j}^{\prime}<\sum_{j \in S} I\left(U_{j} ; U_{S[j] \cup S^{c}}, \hat{Y}_{T^{c}}, Y_{N}\right)+I\left(X_{1} ; \hat{Y}_{T^{c}}, Y_{N} \mid U_{[2: N-1]}\right) \\
&+\sum_{j \in T} I\left(\hat{Y}_{j} ; X_{1}, U_{[2: N-1]}, \hat{Y}_{T[j] \cup T^{c}}, Y_{N} \mid U_{j}\right)
\end{aligned}
$$

for all $k \in[2: N]$ and for all $S$ and $T$ such that $S \subseteq T \subseteq[2: N-1]$. By performing the F-M elimination, Theorem 3 is proved.

Now, let us show that the GDCAF scheme strictly outperforms many existing schemes for the diamond network illustrated in Fig. 7 In this diamond network, $X_{1}=\left(X_{1}^{\prime}, X_{1}^{\prime \prime}\right)$, the channel from $X_{1}^{\prime}$ to $Y_{2}$ is a binary erasure channel (BEC) with erasure probability $\varepsilon$, and the channel from $X_{1}^{\prime \prime}$ to $Y_{3}$ is a binary symmetric channel (BSC) with cross over probability $\varepsilon$, where the two channels are correlated, i.e., cross over in the BSC happens iff an erasure occurs in the BEC. We have $X_{2}=\left(X_{2}^{\prime}, X_{2}^{\prime \prime}\right)$ and $Y_{4}=X_{2}^{\prime}+\left(X_{2}^{\prime \prime} \oplus X_{3}\right)$, where $X_{2}^{\prime}, X_{2}^{\prime \prime}$, and $X_{3}$ are binary and $\oplus$ denotes the XOR operation. Let us assume $\varepsilon=1-H(1 / 3)$. Then, the capacity of the BEC is $1-\varepsilon \approx 0.9183$ and the capacity of the BSC is $1-H(\varepsilon) \approx 0.5918$. The cut-set bound is given as $\log 3 \approx 1.5850$.

In this diamond network, the GDCAF scheme achieves the cut-set bound, which means the capacity is $\log 3$. Let $\hat{Y}_{2}=\hat{Y}_{3}=U_{3}=\emptyset, U_{2}=\left(X_{1}^{\prime}, X_{2}^{\prime}\right), X_{1}^{\prime} \sim \operatorname{Bern}(1 / 2),\left(X_{1}^{\prime \prime}, X_{2}^{\prime}\right) \sim \operatorname{Unif}(\{(0,0),(0,1),(1,1)\})$, $X_{2}^{\prime \prime}=0$ if $Y_{2}$ is not erased, $X_{2}^{\prime \prime}=1$ if $Y_{2}$ is erased, and $X_{3}=Y_{3}$, where $X_{1}^{\prime}$ and $\left(X_{1}^{\prime \prime}, X_{2}^{\prime}\right)$ are independent. 
Then, the GDCAF rate is given as

$$
\min \left\{I\left(X_{1}, X_{2}^{\prime} ; Y_{4}\right), I\left(X_{1} ; Y_{4} \mid X_{1}^{\prime}, X_{2}^{\prime}\right)+I\left(X_{1}^{\prime}, X_{2}^{\prime} ; Y_{2}\right)\right\}=\log 3
$$

Our choice of coding parameters for GDCAF scheme indicates that node 2 performs a combination of partial DF and AF and node 3 employs AF. Now, let us show the suboptimality of DF [52], partial DF, DDF [13], and hybrid coding [12] schemes. First, the DF rate [52] is given as $\min \left\{I\left(X_{1} ; Y_{2}\right), I\left(X_{1} ; Y_{3}\right)\right.$, $\left.I\left(X_{2}, X_{3} ; Y_{4}\right)\right\}$ for some $p\left(x_{1}\right) p\left(x_{2}, x_{3}\right)$, which is upper-bounded by 1 . Next, a partial DF scheme can be constructed by combining Marton coding with common information [53] for the first hop and the optimal scheme [4] for the MAC with common information for the second hop. From the first hop, we have

$$
\begin{aligned}
R & <I\left(U_{0}, U_{1} ; Y_{2}\right)+I\left(U_{2} ; Y_{3} \mid U_{0}\right)-I\left(U_{1} ; U_{2} \mid U_{0}\right) \\
& \leq I\left(U_{0}, U_{1}, X_{1} ; Y_{2}\right)+I\left(U_{2}, X_{1} ; Y_{3} \mid U_{0}\right) \\
& \leq I\left(X_{1} ; Y_{2}\right)+I\left(X_{1} ; Y_{3}\right) \\
& \leq 1-\varepsilon+1-H(\varepsilon) \approx 1.5101
\end{aligned}
$$

for some $p\left(u_{0}, u_{1}, u_{2}\right)$ and a function $x_{1}\left(u_{0}, u_{1}, u_{2}\right)$. Thus, such a partial DF scheme is also suboptimal.

On the other hand, the DDF rate [13] for the diamond channel is given as follows:

$$
\begin{aligned}
& R<\min \{ I\left(X_{1} ; U_{2}, U_{3} \mid X_{2}, X_{3}\right)-I\left(U_{2} ; X_{1}, X_{3} \mid X_{2}, Y_{2}\right)-I\left(U_{3} ; U_{2}, X_{1}, X_{2} \mid X_{3}, Y_{3}\right), \\
& I\left(X_{1}, X_{2} ; U_{3}, Y_{4} \mid X_{3}\right)-I\left(U_{3} ; X_{1}, X_{2} \mid X_{3}, Y_{3}\right), \\
&\left.I\left(X_{1}, X_{3} ; U_{2}, Y_{4} \mid X_{2}\right)-I\left(U_{2} ; X_{1}, X_{3} \mid X_{2}, Y_{2}\right), I\left(X_{2}, X_{3} ; Y_{4}\right)\right\} \\
&=\min \{ H\left(U_{2}, U_{3} \mid X_{2}, X_{3}\right)-H\left(U_{2} \mid X_{2}, Y_{2}\right)-H\left(U_{3} \mid X_{3}, Y_{3}\right), \\
&\left.I\left(X_{2} ; Y_{4} \mid U_{3}, X_{3}\right)+I\left(U_{3} ; Y_{3} \mid X_{3}\right), I\left(X_{3} ; Y_{4} \mid U_{2}, X_{2}\right)+I\left(U_{2} ; Y_{2} \mid X_{2}\right), I\left(X_{2}, X_{3} ; Y_{4}\right)\right\}
\end{aligned}
$$


for $p\left(x_{2}\right) p\left(x_{3}\right) p\left(x_{1}, u_{2}, u_{3} \mid x_{2}, x_{3}\right)$. The DDF rate is upper-bounded as follows:

$$
\begin{aligned}
R & \leq H\left(U_{2}, U_{3} \mid X_{2}, X_{3}\right)-H\left(U_{2} \mid X_{2}, Y_{2}\right)-H\left(U_{3} \mid X_{3}, Y_{3}\right) \\
& \leq H\left(U_{2} \mid X_{2}\right)+H\left(U_{3} \mid X_{3}\right)-H\left(U_{2} \mid X_{2}, Y_{2}\right)-H\left(U_{3} \mid X_{3}, Y_{3}\right) \\
& =I\left(U_{2} ; Y_{2} \mid X_{2}\right)+I\left(U_{3} ; Y_{3} \mid X_{3}\right) \\
& \leq I\left(U_{2}, X_{1} ; Y_{2} \mid X_{2}\right)+I\left(U_{3}, X_{1} ; Y_{3} \mid X_{3}\right) \\
& \leq H\left(Y_{2}\right)-H\left(Y_{2} \mid X_{1}\right)+H\left(Y_{3}\right)-H\left(Y_{3} \mid X_{1}\right) \\
& =I\left(X_{1} ; Y_{2}\right)+I\left(X_{1} ; Y_{3}\right) \\
& \leq 1-\varepsilon+1-H(\varepsilon) \approx 1.5101,
\end{aligned}
$$

hence it is suboptimal.

Lastly, the hybrid coding scheme achieves the following rate

$$
\begin{aligned}
\min \{ & I\left(X_{1} ; \hat{Y}_{2}, \hat{Y}_{3}, Y_{4}\right), I\left(X_{1}, \hat{Y}_{2} ; \hat{Y}_{3}, Y_{4}\right)-I\left(\hat{Y}_{2} ; Y_{2} \mid X_{1}\right), \\
& \left.I\left(X_{1}, \hat{Y}_{3} ; \hat{Y}_{2}, Y_{4}\right)-I\left(\hat{Y}_{3} ; Y_{3} \mid X_{1}\right), I\left(X_{1}, \hat{Y}_{2}, \hat{Y}_{3} ; Y_{4}\right)-I\left(\hat{Y}_{2}, \hat{Y}_{3} ; Y_{2}, Y_{3} \mid X_{1}\right)\right\}
\end{aligned}
$$

for some $p\left(x_{1}\right) p\left(\hat{y}_{2} \mid y_{2}\right) p\left(\hat{y}_{3} \mid y_{3}\right)$ and functions $x_{2}\left(\hat{y}_{2}, y_{2}\right)$ and $x_{3}\left(\hat{y}_{3}, y_{3}\right)$. Let us show the suboptimality of the hybrid coding scheme by contradiction. Assume hybrid coding achieves the capacity. Fix $p\left(x_{1}\right) p\left(\hat{y}_{2} \mid y_{2}\right) p\left(\hat{y}_{3} \mid y_{3}\right)$ and functions $x_{2}\left(\hat{y}_{2}, y_{2}\right)$ and $x_{3}\left(\hat{y}_{3}, y_{3}\right)$ that achieves the capacity. Then

$$
I\left(X_{1}, \hat{Y}_{2}, \hat{Y}_{3} ; Y_{4}\right)-I\left(\hat{Y}_{2}, \hat{Y}_{3} ; Y_{2}, Y_{3} \mid X_{1}\right) \geq \log 3
$$

should hold, which implies

$$
\begin{aligned}
H\left(Y_{4}\right) & =\log 3 \\
H\left(Y_{4} \mid X_{1}, \hat{Y}_{2}, \hat{Y}_{3}\right) & =0 \\
I\left(\hat{Y}_{2}, \hat{Y}_{3} ; Y_{2}, Y_{3} \mid X_{1}\right) & =0 .
\end{aligned}
$$

From (28), we have $p\left(\hat{y}_{2}, \hat{y}_{3} \mid y_{2}, y_{3}, x_{1}\right)=p\left(\hat{y}_{2}, \hat{y}_{3} \mid x_{1}\right)$ for all $\left(y_{2}, y_{3}, x_{1}\right)$ such that $p\left(y_{2}, y_{3}, x_{1}\right)>0$. On the other hand, for all $\left(y_{2}, y_{3}, x_{1}\right)$ such that $p\left(y_{2}, y_{3}, x_{1}\right)>0, p\left(\hat{y}_{2}, \hat{y}_{3} \mid y_{2}, y_{3}, x_{1}\right)=p\left(\hat{y}_{2} \mid y_{2}\right) p\left(\hat{y}_{3} \mid y_{3}\right)$ should hold. Thus, we conclude that $p\left(\hat{y}_{2} \mid y_{2}\right) p\left(\hat{y}_{3} \mid y_{3}\right)=p\left(\hat{y}_{2}, \hat{y}_{3} \mid x_{1}\right)$ for all $\left(y_{2}, y_{3}, x_{1}\right)$ such that $p\left(y_{2}, y_{3}, x_{1}\right)>0$. Note that $p\left(x_{1}\right)>0$ for at least three different values of $x_{1}$ since otherwise the achievable rate is upper bounded by $R \leq H\left(X_{1}\right) \leq 1$. This means that there exists $x \in\{0,1\}$ such that 
$p\left(x_{1}^{\prime}=0, x_{1}^{\prime \prime}=x\right)>0$ and $p\left(x_{1}^{\prime}=1, x_{1}^{\prime \prime}=x\right)>0$. Then, we have

$$
\begin{array}{r}
p\left(\hat{y}_{2} \mid y_{2}=0\right) p\left(\hat{y}_{3} \mid y_{3}=x\right)=p\left(\hat{y}_{2}, \hat{y}_{3} \mid x_{1}^{\prime}=0, x_{1}^{\prime \prime}=x\right) \\
p\left(\hat{y}_{2} \mid y_{2}=E\right) p\left(\hat{y}_{3} \mid y_{3}=1-x\right)=p\left(\hat{y}_{2}, \hat{y}_{3} \mid x_{1}^{\prime}=0, x_{1}^{\prime \prime}=x\right) \\
p\left(\hat{y}_{2} \mid y_{2}=1\right) p\left(\hat{y}_{3} \mid y_{3}=x\right)=p\left(\hat{y}_{2}, \hat{y}_{3} \mid x_{1}^{\prime}=1, x_{1}^{\prime \prime}=x\right) \\
p\left(\hat{y}_{2} \mid y_{2}=E\right) p\left(\hat{y}_{3} \mid y_{3}=1-x\right)=p\left(\hat{y}_{2}, \hat{y}_{3} \mid x_{1}^{\prime}=1, x_{1}^{\prime \prime}=x\right) .
\end{array}
$$

Therefore, we get

$$
p\left(\hat{y}_{2} \mid y_{2}=0\right) p\left(\hat{y}_{3} \mid y_{3}=x\right)=p\left(\hat{y}_{2} \mid y_{2}=E\right) p\left(\hat{y}_{3} \mid y_{3}=1-x\right)=p\left(\hat{y}_{2} \mid y_{2}=1\right) p\left(\hat{y}_{3} \mid y_{3}=x\right)
$$

for all $\hat{y}_{2}$ and $\hat{y}_{3}$. Thus, we conclude $p\left(\hat{y}_{3} \mid y_{3}\right)=p\left(\hat{y}_{3}\right)$ by summing $p\left(\hat{y}_{2} \mid y_{2}=0\right) p\left(\hat{y}_{3} \mid y_{3}=x\right)=$ $p\left(\hat{y}_{2} \mid y_{2}=E\right) p\left(\hat{y}_{3} \mid y_{3}=1-x\right)$ over $\hat{y}_{2}$. Similarly, we conclude $p\left(\hat{y}_{2} \mid y_{2}\right)=p\left(\hat{y}_{2}\right)$. Thus, we get $p\left(x_{1}, y_{2}, y_{3}, \hat{y}_{2}, \hat{y}_{3}\right)=p\left(x_{1}\right) p\left(y_{2}, y_{3} \mid x_{1}\right) p\left(\hat{y}_{2}\right) p\left(\hat{y}_{3}\right)$.

From (27), we have

$$
H\left(Y_{4} \mid X_{1}=x_{1}, \hat{Y}_{2}=\hat{y}_{2}, \hat{Y}_{3}=\hat{y}_{3}\right)=0
$$

for all $\left(x_{1}, \hat{y}_{2}, \hat{y}_{3}\right)$ such that $p\left(x_{1}, \hat{y}_{2}, \hat{y}_{3}\right)=p\left(x_{1}\right) p\left(\hat{y}_{2}\right) p\left(\hat{y}_{3}\right)>0$. On the other hand, since we are assuming hybrid coding achieves the capacity, we must have $I\left(X_{1} ; \hat{Y}_{2}, \hat{Y}_{3}, Y_{4}\right) \geq H\left(Y_{4}\right)=\log 3$. Note that $I\left(X_{1} ; \hat{Y}_{2}, \hat{Y}_{3}, Y_{4}\right)=I\left(X_{1} ; Y_{4} \mid \hat{Y}_{2}, \hat{Y}_{3}\right) \leq H\left(Y_{4} \mid \hat{Y}_{2}, \hat{Y}_{3}\right)$. Thus, we conclude $H\left(Y_{4} \mid \hat{Y}_{2}, \hat{Y}_{3}\right)=H\left(Y_{4}\right)=$ $\log 3$. This means

$$
H\left(Y_{4} \mid \hat{Y}_{2}=\hat{y}_{2}, \hat{Y}_{3}=\hat{y}_{3}\right)=\log 3
$$

for all $\hat{y}_{2}$ and $\hat{y}_{3}$ such that $p\left(\hat{y}_{2}\right) p\left(\hat{y}_{3}\right)>0$.

Because $Y_{4}$ is a function of $X_{2}$ and $X_{3}$, there exists a function $y_{4}(\cdot)$ such that $Y_{4}=y_{4}\left(Y_{2}, Y_{3}, \hat{Y}_{2}, \hat{Y}_{3}\right)$. Fix $\left(\hat{y}_{2}, \hat{y}_{3}\right)$ such that $p\left(\hat{y}_{2}\right) p\left(\hat{y}_{3}\right)>0$. Because $p\left(x_{1}^{\prime}=0, x_{1}^{\prime \prime}=x\right)>0$, we have $p\left(x_{1}^{\prime}=0, x_{1}^{\prime \prime}=x, y_{2}=\right.$ $\left.0, y_{3}=x\right)>0$ and $p\left(x_{1}^{\prime}=0, x_{1}^{\prime \prime}=x, y_{2}=E, y_{3}=1-x\right)>0$. Due to (29), this implies

$$
y_{4}\left(0, x, \hat{y}_{2}, \hat{y}_{3}\right)=y_{4}\left(E, 1-x, \hat{y}_{2}, \hat{y}_{3}\right)
$$

Similarly, from $p\left(x_{1}^{\prime}=1, x_{1}^{\prime \prime}=x\right)>0$, we conclude

$$
y_{4}\left(1, x, \hat{y}_{2}, \hat{y}_{3}\right)=y_{4}\left(E, 1-x, \hat{y}_{2}, \hat{y}_{3}\right) .
$$

Hence, we have

$$
y_{4}\left(0, x, \hat{y}_{2}, \hat{y}_{3}\right)=y_{4}\left(1, x, \hat{y}_{2}, \hat{y}_{3}\right)=y_{4}\left(E, 1-x, \hat{y}_{2}, \hat{y}_{3}\right) .
$$


From (29), (30), and (33), both $p\left(x_{1}^{\prime}=0, x_{1}^{\prime \prime}=1-x\right)$ and $p\left(x_{1}^{\prime}=1, x_{1}^{\prime \prime}=1-x\right)$ should be positive, which implies

$$
y_{4}\left(0,1-x, \hat{y}_{2}, \hat{y}_{3}\right)=y_{4}\left(1,1-x, \hat{y}_{2}, \hat{y}_{3}\right)=y_{4}\left(E, x, \hat{y}_{2}, \hat{y}_{3}\right)
$$

Note that (33) and (34) implies that for $\left(\hat{Y}_{2}, \hat{Y}_{3}\right)=\left(\hat{y}_{2}, \hat{y}_{3}\right), Y_{4}$ has only two possibilities, which is contradictory to (30). Therefore, we conclude that the hybrid coding scheme is strictly suboptimal.

\section{ACYClic GAUSSIAN Network}

In this section, we consider an $N$-node acyclic Gaussian network (AGN), in which the channel output $Y_{k}$ and channel input $X_{k}$ at node $k$ are $r_{k}$-dimensional and $t_{k}$-dimensional vectors, respectively, and the channel from nodes $1, \ldots, k-1$ to node $k$ is given as

$$
Y_{k}=\sum_{j \in[1: k-1]} H_{k j} X_{j}+\sum_{j \in[1: k-1]} H_{k j}^{\prime} Y_{j}+Y_{k}^{\prime}
$$

where $H_{k j}$ is an $r_{k} \times t_{j}$ matrix, $H_{k j}^{\prime}$ is an $r_{k} \times r_{j}$ matrix, and $Y_{k}^{\prime} \sim \mathcal{N}\left(0, \Lambda_{Y_{k}^{\prime}}\right)$ is independent from $X^{k-1}$ and $Y^{k-1}$. Let $n$ denote the number of channel uses and $Y_{k}^{n}$ and $X_{k}^{n}$ for $k \in[1: N]$ denote the $r_{k} \times n$ and $t_{k} \times n$ matrices, respectively, where the $i$-th vectors $Y_{k, i}$ and $X_{k, i}$ of $Y_{k}^{n}$ and $X_{k}^{n}$ are the channel output and channel input, respectively, at node $k$ at the $i$-th channel use. Similarly as in ADMNs, node operations are sequential, i.e., $Y_{1}^{n}$ is generated according to (35) and node 1 maps it to $X_{1}^{n}, Y_{2}^{n}$ is generated according to (35) and node 2 maps it to $X_{2}^{n}$, and so on.

The objective of this network is specified by a $\theta$-dimensional nonnegative vector $\Theta^{*}$ and a nonnegative function $\Theta$ that maps $\left(x_{1}, \ldots, x_{N}, y_{1}, \ldots, y_{N}\right) \in \mathcal{X}_{1} \times \ldots \times \mathcal{X}_{N} \times \mathcal{Y}_{1} \times \ldots \times \mathcal{Y}_{N}$ to a $\theta$-dimensional vector whose elements are all nonnegative. For a set of node processing functions $Y_{k}^{n} \rightarrow X_{k}^{n}, k=1, \ldots, N$, the $\epsilon$-probability of error for $\epsilon>0$ is defined as

$$
P_{e}^{(n)}\left(\Theta, \Theta^{*}, \epsilon\right)=1-P\left(\frac{1}{n} \sum_{i=1}^{n} \Theta\left(X_{1, i}, \ldots, X_{N, i}, Y_{1, i}, \ldots, Y_{N, i}\right) \prec(1+\epsilon) \Theta^{*}\right) .
$$

We say $\left(\Theta, \Theta^{*}\right)$ is achievable if there exists a sequence of node processing functions $Y_{k}^{n} \rightarrow X_{k}^{n}$, $k=1, \ldots, N$, such that $\lim _{n \rightarrow \infty} P_{e}^{(n)}\left(\Theta, \Theta^{*}, \epsilon\right)=0$ for any $\epsilon>0$. We note that $\left(\Theta, \Theta^{*}\right)$ can be used for imposing input power constraint and quadratic distortion constraint between a source sequence and a reconstructed sequence.

To derive a sufficient condition for achieving $\left(\Theta, \Theta^{*}\right)$ by using Theorem 11 let us first assume a distribution $f^{*}\left(x_{[1: N]}, y_{[1: N]}\right)$ such that $\mathrm{E}\left(\Theta\left(X_{1}, \ldots, X_{N}, Y_{1}, \ldots, Y_{N}\right)\right) \prec \Theta^{*}$. For such $f^{*}\left(x_{[1: N]}, y_{[1: N]}\right)$, 
consider a subset $\Omega_{g}\left(f^{*}\right)$ of $\Omega\left(f^{*}\right) 6$ where $U_{j}$ is an $a_{j}$-dimensional vector and $U_{W_{k}}$ and $X_{k}$ have the form of

$$
\begin{gathered}
U_{W_{k}}=G_{k} U_{D_{k}}+G_{k}^{\prime} Y_{k}+U_{W_{k}}^{\prime} \\
X_{k}=F_{k} U_{D_{k} \cup W_{k}}+F_{k}^{\prime} Y_{k} .
\end{gathered}
$$

In the above, $G_{k}$ is a $\sum_{j \in W_{k}} a_{j} \times \sum_{j \in D_{k}} a_{j}$ matrix, $G_{k}^{\prime}$ is a $\sum_{j \in W_{k}} a_{j} \times r_{k}$ matrix, $U_{W_{k}}^{\prime} \sim \mathcal{N}\left(0, \Lambda_{U_{W_{k}}^{\prime}}\right)$ is a $\sum_{j \in W_{k}} a_{j}$-dimensional Gaussian vector, $F_{k}$ is a $t_{k} \times \sum_{j \in D_{k} \cup W_{k}} a_{j}$ matrix, and $F_{k}^{\prime}$ is a $t_{k} \times r_{k}$ matrix, where $U_{W_{k}}^{\prime}$ is independent from $U_{D_{k}}$ and $Y_{k}$.

Note that $U_{W_{k}}$ and $X_{k}$ can be rewritten as follows:

$$
\begin{aligned}
U_{W_{k}} & =\sum_{j \in[1: k-1]} G_{k j} U_{W_{j}}+G_{k}^{\prime} Y_{k}+U_{W_{k}}^{\prime} \\
X_{k} & =\sum_{j \in[1: k]} F_{k j} U_{W_{j}}+F_{k}^{\prime} Y_{k},
\end{aligned}
$$

where the columns of $G_{k j}$ and $F_{k j}$ corresponding to $U_{D_{k}}$ and $U_{D_{k} \cup W_{k}}$ are from $G_{k}$ and $F_{k}$, respectively, and the other columns of $G_{k j}$ and $F_{k j}$ are zero vectors.

The following lemma gives $U_{W_{k}}$ and $Y_{k}$, whose proof is given at the end of this section.

Lemma 2: For $k \in[1: N]$, we have

$$
\left[\begin{array}{c}
U_{W_{k}} \\
Y_{k}
\end{array}\right]=\sum_{j \in[1: k]} \Phi_{k j} \Psi_{j}
$$

where

$$
\begin{gathered}
\Phi_{k j} \triangleq\left\{\begin{array}{cc}
\sum_{S:\{j, k\} \subseteq S \subseteq[j: k]} \prod_{i \in[1:|S|-1]} \Upsilon_{S_{[i+1]} S_{[i]}} & \text { if } j<k \\
\mathbf{I} & \text { if } j=k
\end{array},\right. \\
\Psi_{j} \triangleq\left[\begin{array}{c}
G_{j}^{\prime} Y_{j}^{\prime}+U_{W_{j}}^{\prime} \\
Y_{j}^{\prime}
\end{array}\right], \\
\Upsilon_{k^{\prime} j^{\prime}} \triangleq\left[\begin{array}{cc}
G_{k^{\prime} j^{\prime}}+G_{k^{\prime}}^{\prime} \sum_{i \in\left[j^{\prime}: k^{\prime}-1\right]} H_{k^{\prime} i} F_{i j^{\prime}} & G_{k^{\prime}}^{\prime}\left(H_{k^{\prime} j^{\prime}} F_{j^{\prime}}^{\prime}+H_{k^{\prime} j^{\prime}}^{\prime}\right) \\
\sum_{i \in\left[j^{\prime}: k^{\prime}-1\right]} H_{k^{\prime} i} F_{i j^{\prime}} & H_{k^{\prime} j^{\prime}} F_{j^{\prime}}^{\prime}+H_{k^{\prime} j^{\prime}}^{\prime}
\end{array}\right] .
\end{gathered}
$$

From Lemma 2] for any $S \subseteq W^{N}$, we can construct a matrix $\Phi_{U_{S}}$ such that $U_{S}=\Phi_{U_{S}} \Psi^{k(S)}$, where $k(S)=\max \left(\left\{k: S \cap W_{k} \neq \emptyset\right\}\right)$. Also, for any $k \in[1: N]$ and $S \subseteq W^{k}$, we can construct matrices $\Phi_{U_{S}, Y_{k}}$ such that $\left[U_{S}^{t} Y_{k}^{t}\right]^{t}=\Phi_{U_{S}, Y_{k}} \Psi^{k}$.

\footnotetext{
${ }^{6}$ The definition of $\Omega$ in Section $\Pi$ can be naturally generalized for continous random variables.
} 
Now, we are ready to present a sufficient condition for achieving $\left(\Theta, \Theta^{*}\right)$ for an $N$-node AGN.

Theorem 4: For an $N$-node AGN, $\left(\Theta, \Theta^{*}\right)$ is achievable if there exists $\omega_{g} \in \Omega_{g}\left(f^{*}\right)$ for some $f^{*}$ that satisfies $\mathrm{E}\left(\Theta\left(X_{1}, \ldots, X_{N}, Y_{1}, \ldots, Y_{N}\right)\right) \prec \Theta^{*}$ such that for $1 \leq k \leq N$

$$
\begin{aligned}
& \sum_{j \in \bar{S}_{k}} r_{j}<\frac{1}{2} \log \frac{\left|\Phi_{U_{S_{k}^{c}}, Y_{k}} \Lambda_{\Psi^{k}} \Phi_{U_{S_{k}^{c}}, Y_{k}}^{t}\right| \cdot \prod_{j \in S_{k}}\left|\Phi_{U_{j}, U_{A_{j}}} \Lambda_{\Psi^{k\left(\{j\} \cup A_{j}\right)}} \Phi_{U_{j}, U_{A_{j}}}^{t}\right|}{\left|\Phi_{U_{D_{k} \cup B_{k}}, Y_{k}} \Lambda_{\Psi^{k}} \Phi_{U_{D_{k} \cup B_{k}}, Y_{k}}^{t}\right| \cdot \prod_{j \in S_{k}}\left|\Phi_{U_{A_{j}}} \Lambda_{\Psi^{k\left(A_{j}\right)}} \Phi_{U_{A_{j}}}^{t}\right|} \\
& \sum_{j \in \bar{T}_{k}} r_{j}>\frac{1}{2} \log \frac{\prod_{j \in T_{k}}\left|\Phi_{U_{j}, U_{A_{j}}} \Lambda_{\Psi^{k\left(\{j\} \cup A_{j}\right)}} \Phi_{U_{j}, U_{A_{j}}}^{t}\right|}{\left|\Lambda_{U_{T_{k}}^{\prime}}\right| \cdot \prod_{j \in T_{k}}\left|\Phi_{U_{A_{j}}} \Lambda_{\Psi^{k\left(A_{j}\right)}} \Phi_{U_{A_{j}}}^{t}\right|}
\end{aligned}
$$

for all $\bar{S}_{k} \subseteq \bar{D}_{k} \cup \bar{B}_{k}$ such that $\bar{S}_{k} \cap \bar{D}_{k} \neq \emptyset$ and for all $\bar{T}_{k} \subseteq \bar{W}_{k}$ such that $\bar{T}_{k} \neq \emptyset$, where $\bar{D}_{k}, \bar{B}_{k}, \bar{W}_{k}$, $S_{k}$, and $T_{k}$ are defined in Theorem 1 and $\Lambda_{\Psi^{k}}$ is a block diagonal matrix with diagonal blocks

$$
\Lambda_{\Psi_{j}}=\left[\begin{array}{cc}
\Lambda_{U_{W_{j}}^{\prime}}+G_{j}^{\prime} \Lambda_{Y_{j}^{\prime}} G_{j}^{\prime t} & G_{j}^{\prime} \Lambda_{Y_{j}^{\prime}} \\
\Lambda_{Y_{j}^{\prime}} G_{j}^{\prime t} & \Lambda_{Y_{j}^{\prime}}
\end{array}\right], \quad j \in[1: k] .
$$

Proof: We apply Theorem 1 for $f^{*}\left(x_{[1: N]}, y_{[1: N]}\right)$ such that $\mathrm{E}\left(\Theta\left(X_{1}, \ldots, X_{N}, Y_{1}, \ldots, Y_{N}\right)\right) \prec \Theta^{*}$ and $\omega_{g} \in \Omega_{g}\left(f^{*}\right)$. Then, the inequalities in Theorem 1 is given as follows: for $k \in[1: N], \bar{S}_{k} \subseteq \bar{D}_{k} \cup \bar{B}_{k}$ such that $\bar{S}_{k} \cap \bar{D}_{k} \neq \emptyset$, and $\bar{T}_{k} \subseteq \bar{W}_{k}$ such that $\bar{T}_{k} \neq \emptyset$, we have

$$
\begin{aligned}
\sum_{j \in \bar{S}_{k}} r_{j} & <\sum_{j \in S_{k}} I\left(U_{j} ; U_{S_{k}[j] \cup S_{k}^{c}}, Y_{k} \mid U_{A_{j}}\right) \\
& =\sum_{j \in S_{k}} h\left(U_{j} \mid U_{A_{j}}\right)-h\left(U_{S_{k}} \mid U_{S_{k}^{c}}, Y_{k}\right) \\
& =\sum_{j \in S_{k}}\left(h\left(U_{j}, U_{A_{j}}\right)-h\left(U_{A_{j}}\right)\right)-h\left(U_{D_{k} \cup B_{k}}, Y_{k}\right)+h\left(U_{S_{k}^{c}}, Y_{k}\right) \\
& =\frac{1}{2} \log \frac{\left|\Phi_{U_{S_{k}^{c}}, Y_{k}} \Lambda_{\Psi^{k}} \Phi_{U_{S_{k}^{c}}, Y_{k}}^{t}\right| \cdot \prod_{j \in S_{k}}\left|\Phi_{U_{j}, U_{A_{j}}} \Lambda_{\Psi_{\Psi^{k\left(\{j\} A_{j}\right)}} \Phi_{U_{j}, U_{A_{j}}}^{t} \mid}\right|}{\left|\Phi_{{D_{k} \cup B_{k}}_{k}, Y_{k}} \Lambda_{\Psi^{k}} \Phi_{U_{D_{k} \cup B_{k}}, Y_{k}}\right| \cdot \prod_{j \in S_{k}}\left|\Phi_{U_{A_{j}}} \Lambda_{\Psi^{k\left(A_{j}\right)}} \Phi_{U_{A_{j}}}^{t}\right|}
\end{aligned}
$$

and

$$
\begin{aligned}
\sum_{j \in \bar{T}_{k}} r_{j}>\sum_{j \in T_{k}} I\left(U_{j} ; U_{T_{k}[j] \cup D_{k}}, Y_{k} \mid U_{A_{j}}\right) \\
=\sum_{j \in T_{k}} h\left(U_{j} \mid U_{A_{j}}\right)-h\left(U_{T_{k}} \mid U_{D_{k}}, Y_{k}\right) \\
=\sum_{j \in T_{k}}\left(h\left(U_{j}, U_{A_{j}}\right)-h\left(U_{A_{j}}\right)\right)-h\left(U_{T_{k}}^{\prime}\right) \\
=\frac{1}{2} \log \frac{\prod_{j \in T_{k}}\left|\Phi_{U_{j}, U_{A_{j}}} \Lambda_{\Psi^{k\left(\{j\} \cup A_{j}\right)}} \Phi_{U_{j}, U_{A_{j}}}^{t}\right|}{\left|\Lambda_{U_{T_{k}}^{\prime}}\right| \cdot \prod_{j \in T_{k}}\left|\Phi_{U_{A_{j}}} \Lambda_{\Psi^{k\left(A_{j}\right)}} \Phi_{U_{A_{j}}}^{t}\right|} .
\end{aligned}
$$

Now, by following the standard discretization procedure [54] and the typical average lemma [41], Theorem 4 is proved. 
Remark 7: If $U_{j}, j \in[1: \nu]$ has the form of $G_{j}^{\prime \prime} U_{A_{j}}+U_{j}^{\prime \prime}$ as a special case of (36) for some matrix $G_{j}^{\prime \prime}$, where $U_{j}^{\prime \prime}$ is independent of $U_{A_{j}}$, then we have

$$
\frac{\left|\Phi_{U_{j}, U_{A_{j}}} \Lambda_{\Psi^{k\left(\{j\} \cup A_{j}\right)}} \Phi_{U_{j}, U_{A_{j}}}^{t}\right|}{\left|\Phi_{U_{A_{j}}} \Lambda_{\Psi^{k\left(A_{j}\right)}} \Phi_{U_{A_{j}}}^{t}\right|}=\left|\Lambda_{U_{j}^{\prime \prime}}\right| .
$$

Proof of Lemma 2. By substituting (38) into (35), $Y_{k}$ is written as follows:

$$
\begin{aligned}
Y_{k} & =\sum_{i \in[1: k-1]} H_{k i}\left(\sum_{j \in[1: i]} F_{i j} U_{W_{j}}+F_{i}^{\prime} Y_{i}\right)+\sum_{j \in[1: k-1]} H_{k j}^{\prime} Y_{j}+Y_{k}^{\prime} \\
& =\sum_{i \in[1: k-1]} \sum_{j \in[1: i]} H_{k i} F_{i j} U_{W_{j}}+\sum_{j \in[1: k-1]}\left(H_{k j} F_{j}^{\prime}+H_{k j}^{\prime}\right) Y_{j}+Y_{k}^{\prime} \\
& \stackrel{(a)}{=} \sum_{j \in[1: k-1]} \sum_{i \in[j: k-1]} H_{k i} F_{i j} U_{W_{j}}+\sum_{j \in[1: k-1]}\left(H_{k j} F_{j}^{\prime}+H_{k j}^{\prime}\right) Y_{j}+Y_{k}^{\prime},
\end{aligned}
$$

where $(a)$ is by changing the summation order.

Next, by substituting (43) into (37), $U_{W_{k}}$ is given as follows:

$$
\begin{aligned}
U_{W_{k}} & =\sum_{j \in[1: k-1]} G_{k j} U_{W_{j}}+G_{k}^{\prime}\left(\sum_{j \in[1: k-1]} \sum_{i \in[j: k-1]} H_{k i} F_{i j} U_{W_{j}}+\sum_{j \in[1: k-1]}\left(H_{k j} F_{j}^{\prime}+H_{k j}^{\prime}\right) Y_{j}+Y_{k}^{\prime}\right)+U_{W_{k}}^{\prime} \\
& =\sum_{j \in[1: k-1]}\left(G_{k j}+G_{k}^{\prime} \sum_{i \in[j: k-1]} H_{k i} F_{i j}\right) U_{W_{j}}+\sum_{j \in[1: k-1]} G_{k}^{\prime}\left(H_{k j} F_{j}^{\prime}+H_{k j}^{\prime}\right) Y_{j}+G_{k}^{\prime} Y_{k}^{\prime}+U_{W_{k}}^{\prime} .
\end{aligned}
$$

Hence, we have

$$
\left[\begin{array}{c}
U_{W_{k}} \\
Y_{k}
\end{array}\right]=\sum_{j \in[1: k-1]} \Upsilon_{k j}\left[\begin{array}{c}
U_{W_{j}} \\
Y_{j}
\end{array}\right]+\Psi_{k}
$$

where $\Upsilon_{k j}$ and $\Psi_{k}$ are defined in (42) and (41), respectively.

We prove Lemma 2 by solving the recursive formula in (44) using strong induction. For $k=1$, $\left[\begin{array}{ll}U_{W_{1}}^{t} & Y_{1}^{t}\end{array}\right]^{t}=\Psi_{1}$ from (44), and hence Lemma 2 holds trivially. For $k>1$, assume that $\left[U_{W_{j}}^{t} Y_{j}^{t}\right]^{t}=$ $\sum_{i \in[1: j]} \Phi_{j i} \Psi_{i}$ for all $j<k$. Then,

$$
\begin{aligned}
& {\left[\begin{array}{ll}
U_{W_{k}}^{t} & Y_{k}^{t}
\end{array}\right]^{t} \stackrel{(a)}{=} \sum_{j \in[1: k-1]} \Upsilon_{k j}\left[U_{W_{j}}^{t} Y_{j}^{t}\right]^{t}+\Psi_{k}} \\
& \stackrel{(b)}{=} \sum_{j \in[1: k-1]} \sum_{i \in[1: j]} \Upsilon_{k j} \Phi_{j i} \Psi_{i}+\Psi_{k} \\
& \stackrel{(c)}{=} \sum_{i \in[1: k-1]} \sum_{j \in[i: k-1]} \Upsilon_{k j} \Phi_{j i} \Psi_{i}+\Psi_{k},
\end{aligned}
$$


where $(a)$ is from (44), $(b)$ is from the induction assumption, and $(c)$ is by changing the summation order. Now, we have

$$
\begin{aligned}
\sum_{j \in[i: k-1]} \Upsilon_{k j} \Phi_{j i} & =\Upsilon_{k i}+\sum_{j \in[i+1: k-1]} \Upsilon_{k j} \Phi_{j i} \\
& =\Upsilon_{k i}+\sum_{j \in[i+1: k-1]} \Upsilon_{k j} \sum_{S:\{i, j\} \subseteq S \subseteq[i: j]} \prod_{i^{\prime} \in[1:|S|-1]} \Upsilon_{S_{\left[i^{\prime}+1\right]} S_{\left[i^{\prime}\right]}} \\
& =\sum_{S:\{i, k\} \subseteq S \subseteq[i: k] i^{\prime} \in[1:|S|-1]} \Upsilon_{S_{\left[i^{\prime}+1\right]} S_{\left[i^{\prime}\right]}} \\
& =\Phi_{k i}
\end{aligned}
$$

Hence, we have $\left[U_{W_{k}}^{t} Y_{k}^{t}\right]^{t}=\sum_{j \in[1: k]} \Phi_{k j} \Psi_{j}$, and Lemma 2 is proved by strong induction.

\section{CONCLUSION}

We showed a unified achievability theorem that generalizes most of achievability results in network information theory that are based on random coding. Our single-letter rate expression has a very simple form. This was made possible due to our framework, where many different ingredients in network information theory are treated in a unified way, and our coding scheme that consists of a few basic ingredients but is at least as powerful as many existing schemes. Using our result, obtaining many new achievability results in network information theory can now be done more easily. As a simple application of our main theorem, we derived a generalized decode-compress-amplify-and-forward bound and showed it strictly outperforms previously known results. Because the final expression of our main theorem has a simple form, it enables us to get new insights. As an example, we showed how to derive three types of network duality from our main theorem. Our result can be made more general if other coding strategies such as structured codes can also be incorporated in our setting. However, such a task does not seem easy and the rate expression may become too complicated. 


\section{APPENDIX}

A. Bounding the second term in the summation in (8)

For given $k \in[1: N]$, we have

$$
\begin{aligned}
& P\left(\mathcal{E}_{k, 2} \cap \bigcap_{j=1}^{k-1}\left(\mathcal{E}_{j, 1} \cup \mathcal{E}_{j, 2} \cup \mathcal{E}_{j, 3}\right)^{c}\right) \\
& \leq P\left(\left(U_{D_{k} \cup B_{k}}^{n}\left(l_{\bar{D}_{k} \cup \bar{B}_{k}}^{\prime}\right), Y_{k}^{n}\right) \in \mathcal{T}_{\epsilon_{k}}^{(n)} \text { for some } l_{\bar{D}_{k} \cup \bar{B}_{k}}^{\prime} \text { s.t. } l_{\bar{D}_{k}}^{\prime} \neq L_{\bar{D}_{k}}, \hat{L}_{\bar{D}_{j}, j}=L_{\bar{D}_{j}},\right. \\
& \left.\left(U_{D_{j} \cup B_{j}}^{n}\left(L_{\bar{D}_{j}}, L_{\bar{B}_{j}}\right), Y_{j}^{n}\right) \in \mathcal{T}_{\epsilon_{j}}^{(n)},\left(U_{D_{j}}^{n}\left(L_{\bar{D}_{j}}\right), U_{W_{j}}^{n}\left(L_{\bar{D}_{j}}, L_{\bar{W}_{j}}\right), Y_{j}^{n}\right) \in \mathcal{T}_{\epsilon_{j}^{\prime}}^{(n)} \text { for all } j \in[1: k-1]\right) \\
& \leq \sum_{\substack{\bar{S}_{k} \subseteq \bar{D}_{k} \cup \bar{B}_{k} \\
\bar{S}_{k} \cap \bar{D}_{k} \neq \emptyset}} P\left(\left(U_{D_{k} \cup B_{k}}^{n}\left(l_{\bar{S}_{k}}^{\prime}, L_{\bar{S}_{k}^{c}}\right), Y_{k}^{n}\right) \in \mathcal{T}_{\epsilon_{k}}^{(n)} \text { for some } l_{\bar{S}_{k}}^{\prime} \text { s.t. } l_{i}^{\prime} \neq L_{i} \text { for all } i \in \bar{S}_{k}, \hat{L}_{\bar{D}_{j}, j}=L_{\bar{D}_{j}},\right. \\
& \left.\left(U_{D_{j} \cup B_{j}}^{n}\left(L_{\bar{D}_{j}}, L_{\bar{B}_{j}}\right), Y_{j}^{n}\right) \in \mathcal{T}_{\epsilon_{j}}^{(n)},\left(U_{D_{j}}^{n}\left(L_{\bar{D}_{j}}\right), U_{W_{j}}^{n}\left(L_{\bar{D}_{j}}, L_{\bar{W}_{j}}\right), Y_{j}^{n}\right) \in \mathcal{T}_{\epsilon_{j}^{\prime}}^{(n)} \text { for all } j \in[1: k-1]\right) .
\end{aligned}
$$

For $\bar{S}_{k} \subseteq \bar{D}_{k} \cup \bar{B}_{k}$ such that $\bar{S}_{k} \cap \bar{D}_{k} \neq \emptyset$, we have

$$
\begin{aligned}
& P\left(\left(U_{D_{k} \cup B_{k}}^{n}\left(l_{\bar{S}_{k}}^{\prime}, L_{\bar{S}_{k}^{c}}\right), Y_{k}^{n}\right) \in \mathcal{T}_{\epsilon_{k}}^{(n)} \text { for some } l_{\bar{S}_{k}}^{\prime} \text { s.t. } l_{i}^{\prime} \neq L_{i} \text { for all } i \in \bar{S}_{k}, \hat{L}_{\bar{D}_{j}, j}=L_{\bar{D}_{j}},\right. \\
&\left.\left(U_{D_{j} \cup B_{j}}^{n}\left(L_{\bar{D}_{j}}, L_{\bar{B}_{j}}\right), Y_{j}^{n}\right) \in \mathcal{T}_{\epsilon_{j}}^{(n)},\left(U_{D_{j}}^{n}\left(L_{\bar{D}_{j}}\right), U_{W_{j}}^{n}\left(L_{\bar{D}_{j}}, L_{\bar{W}_{j}}\right), Y_{j}^{n}\right) \in \mathcal{T}_{\epsilon_{j}^{\prime}}^{(n)} \text { for all } j \in[1: k-1]\right) \\
&=P\left(\left(U_{D_{k} \cup B_{k}}^{n}\left(l_{\bar{S}_{k}}^{\prime}, l_{\bar{S}_{k}^{c}}\right), Y_{k}^{n}\right) \in \mathcal{T}_{\epsilon_{k}}^{(n)} \text { for some } l_{\bar{S}_{k}}^{\prime} \text { s.t. } l_{i}^{\prime} \neq l_{i} \text { for all } i \in \bar{S}_{k}, \hat{L}_{\bar{D}_{j}, j}=l_{\bar{D}_{j}},\right.\left(U_{D_{j} \cup B_{j}}^{n}\left(l_{\bar{D}_{j}}, l_{\bar{B}_{j}}\right), Y_{j}^{n}\right) \in \mathcal{T}_{\epsilon_{j}}^{(n)},\left(U_{D_{j}}^{n}\left(l_{\bar{D}_{j}}\right), U_{W_{j}}^{n}\left(l_{\bar{D}_{j}}, l_{\bar{W}_{j}}\right), Y_{j}^{n}\right) \in \mathcal{T}_{\epsilon_{j}^{\prime}}^{(n)} \text { for all } j \in[1: k-1], \\
&\left.L_{\bar{W}^{k-1}}=l_{\bar{W}^{k-1}} \text { for some } l_{\bar{W}^{k-1}}\right) \\
&=P\left(\left(U_{D_{k} \cup B_{k}}^{n}\left(l_{\bar{S}_{k}}^{\prime}, l_{\bar{S}_{k}^{c}}\right), Y_{k}^{n}\right) \in \mathcal{T}_{\epsilon_{k}}^{(n)} \text { for some } l_{\bar{S}_{k}}^{\prime} \text { s.t. } l_{i}^{\prime} \neq l_{i} \text { for all } i \in \bar{S}_{k}, U_{D_{j} \cup B_{j}}^{n}(\cdot) \in \mathcal{A}_{j}\left(Y_{j}^{n}, l_{\bar{D}_{j} \cup \bar{B}_{j}}\right),\right. \\
&\left.\quad\left(U_{D_{j}}^{n}\left(l_{\bar{D}_{j}}\right), U_{W_{j}}^{n}\left(l_{\bar{D}_{j}}, \cdot\right)\right) \in \mathcal{B}_{j}\left(Y_{j}^{n}, l_{\bar{D}_{j} \cup \bar{W}_{j}}\right) \text { for all } j \in[1: k-1] \text { for some } l_{\bar{W}^{k-1}}\right)
\end{aligned}
$$

where $\mathcal{A}_{j}\left(y_{j}^{n}, l_{\bar{D}_{j} \cup \bar{B}_{j}}\right)$ is the ensemble of codebooks $u_{D_{j} \cup B_{j}}^{n}(\cdot)$ such that node $j$ with received channel output $y_{j}^{n}$ decodes $\hat{l}_{\bar{D}_{j}, j}$ as $l_{\bar{D}_{j}}$ and the joint typicality (6) is satisfied for given $l_{\bar{D}_{j} \cup \bar{B}_{j}}$ and $\mathcal{B}_{j}\left(y_{j}^{n}, l_{\bar{D}_{j} \cup \bar{W}_{j}}\right)$ is the ensemble of codebooks $\left(u_{D_{j}}^{n}\left(l_{\bar{D}_{j}}\right), u_{W_{j}}^{n}\left(l_{\bar{D}_{j}}, \cdot\right)\right)$ such that node $j$ with received channel output $y_{j}^{n}$ and decoded index vector $l_{\bar{D}_{j}}$ chooses $l_{\bar{W}_{j}}$ and the joint typicality (7) is satisfied for given $l_{\bar{D}_{j} \cup \bar{W}_{j}}$. More precisely, we define

$$
\begin{gathered}
\mathcal{A}_{j}\left(y_{j}^{n}, l_{\bar{D}_{j} \cup \bar{B}_{j}}\right) \triangleq\left\{u_{D_{j} \cup B_{j}}^{n}(\cdot):\left(u_{D_{j} \cup B_{j}}^{n}\left(\tilde{l}_{\bar{D}_{j} \cup \bar{B}_{j}}\right), y_{j}^{n}\right) \notin T_{\epsilon_{j}} \text { for all } \tilde{l}_{\bar{D}_{j}}<l_{\bar{D}_{j}}, \tilde{l}_{\bar{B}_{j}},\right. \\
\left.\left(u_{D_{j} \cup B_{j}}^{n}\left(l_{\bar{D}_{j} \cup \bar{B}_{j}}\right), y_{j}^{n}\right) \in T_{\epsilon_{j}}\right\} \\
\mathcal{B}_{j}\left(y_{j}^{n}, l_{\bar{D}_{j} \cup \bar{W}_{j}}\right) \triangleq\left\{\left(u_{D_{j}}^{n}\left(l_{\bar{D}_{j}}\right), u_{W_{j}}^{n}\left(l_{\bar{D}_{j}}, \cdot\right)\right):\left(u_{D_{j}}^{n}\left(l_{\bar{D}_{j}}\right), u_{W_{j}}^{n}\left(l_{\bar{D}_{j}}, \tilde{l}_{\bar{W}_{j}}\right), y_{j}^{n}\right) \notin T_{\epsilon_{j}^{\prime}} \text { for all } \tilde{l}_{\bar{W}_{j}}<l_{\bar{W}_{j}},\right. \\
\left.\left(u_{D_{j}}^{n}\left(l_{\bar{D}_{j}}\right), u_{W_{j}}^{n}\left(l_{\bar{D}_{j} \cup \bar{W}_{j}}\right), y_{j}^{n}\right) \in T_{\epsilon_{j}^{\prime}}\right\} .
\end{gathered}
$$


Continuing with the bound in 46, we have

$P\left(\left(U_{D_{k} \cup B_{k}}^{n}\left(l_{\bar{S}_{k}}^{\prime}, l_{\bar{S}_{k}^{c}}\right), Y_{k}^{n}\right) \in \mathcal{T}_{\epsilon_{k}}^{(n)}\right.$ for some $l_{\bar{S}_{k}}^{\prime}$ s.t. $l_{i}^{\prime} \neq l_{i}$ for all $i \in \bar{S}_{k}, U_{D_{j} \cup B_{j}}^{n}(\cdot) \in \mathcal{A}_{j}\left(Y_{j}^{n}, l_{\bar{D}_{j} \cup \bar{B}_{j}}\right)$,

$\left(U_{D_{j}}^{n}\left(l_{\bar{D}_{j}}\right), U_{W_{j}}^{n}\left(l_{\bar{D}_{j}}, \cdot\right)\right) \in \mathcal{B}_{j}\left(Y_{j}^{n}, l_{\bar{D}_{j} \cup \bar{W}_{j}}\right)$ for all $j \in[1: k-1]$ for some $\left.l_{\bar{W}^{k-1}}\right)$

$=P\left(\left(U_{D_{k} \cup B_{k}}^{n}\left(l_{\bar{S}_{k}}^{\prime}, l_{\bar{S}_{k}^{c}}\right), Y_{k}^{n}\right) \in \mathcal{T}_{\epsilon_{k}}^{(n)}, U_{D_{j} \cup B_{j}}^{n}(\cdot) \in \mathcal{A}_{j}\left(Y_{j}^{n}, l_{\bar{D}_{j} \cup \bar{B}_{j}}\right),\left(U_{D_{j}}^{n}\left(l_{\bar{D}_{j}}\right), U_{W_{j}}^{n}\left(l_{\bar{D}_{j}}, \cdot\right)\right) \in \mathcal{B}_{j}\left(Y_{j}^{n}, l_{\bar{D}_{j} \cup \bar{W}_{j}}\right)\right.$

for all $j \in[1: k-1]$ for some $l_{\bar{W}^{k-1}}$ s.t. $l_{i} \neq l_{i}^{\prime}$ for all $i \in \bar{S}_{k}$ for some $l_{\bar{S}_{k}}^{\prime}$ )

$\leq \sum_{l_{\bar{S}_{k}}^{\prime}} P\left(\left(U_{D_{k} \cup B_{k}}^{n}\left(l_{\bar{S}_{k}}^{\prime}, l_{\bar{S}_{k}^{c}}\right), Y_{k}^{n}\right) \in \mathcal{T}_{\epsilon_{k}}^{(n)}, U_{D_{j} \cup B_{j}}^{n}(\cdot) \in \mathcal{A}_{j}\left(Y_{j}^{n}, l_{\bar{D}_{j} \cup \bar{B}_{j}}\right)\right.$,

$\left(U_{D_{j}}^{n}\left(l_{\bar{D}_{j}}\right), U_{W_{j}}^{n}\left(l_{\bar{D}_{j}}, \cdot\right)\right) \in \mathcal{B}_{j}\left(Y_{j}^{n}, l_{\bar{D}_{j} \cup \bar{W}_{j}}\right)$ for all $j \in[1: k-1]$ for some $l_{\bar{W}^{k-1}}$ s.t. $l_{i} \neq l_{i}^{\prime}$ for all $\left.i \in \bar{S}_{k}\right)$

$\stackrel{(a)}{=} \sum_{l_{\bar{S}_{k}}^{\prime}} P\left(\left(U_{D_{k} \cup B_{k}}^{n}\left(l_{\bar{S}_{k}}^{\prime}, L_{\bar{S}_{k}^{c}}^{\prime \prime}\right), \tilde{Y}_{k}^{n}\right) \in \mathcal{T}_{\epsilon_{k}}^{(n)}, U_{D_{j} \cup B_{j}}^{n}(\cdot) \in \mathcal{A}_{j}\left(Y_{j}^{n}, l_{\bar{D}_{j} \cup \bar{B}_{j}}\right)\right.$,

$\left(U_{D_{j}}^{n}\left(l_{\bar{D}_{j}}\right), U_{W_{j}}^{n}\left(l_{\bar{D}_{j}}, \cdot\right)\right) \in \mathcal{B}_{j}\left(Y_{j}^{n}, l_{\bar{D}_{j} \cup \bar{W}_{j}}\right)$ for all $j \in[1: k-1]$ for some $l_{\bar{W}^{k-1}}$ s.t. $l_{i} \neq l_{i}^{\prime}$ for all $\left.i \in \bar{S}_{k}\right)$

where $\tilde{Y}_{k}^{n}$ is the channel output sequence at node $k$ assuming that decoded index vector $\hat{l}_{\bar{D}_{j}, j}^{\prime \prime}=$ $\hat{l}_{\bar{D}_{j}, j}^{\prime \prime}\left(y_{j}^{n}, l_{\bar{S}_{k}}^{\prime}, u_{D_{j} \cup B_{j}}^{n}\left(\tilde{l}_{\bar{D}_{j} \cup \bar{B}_{j}}\right)\right.$ for all $\tilde{l}_{\bar{D}_{j} \cup \bar{B}_{j}}$ such that $\tilde{l}_{i} \neq l_{i}^{\prime}$ for all $\left.i \in \bar{S}_{k} \cap\left(\bar{D}_{j} \cup \bar{B}_{j}\right)\right)$ and covering index vector $l_{\bar{W}_{j}}^{\prime \prime}=l_{\bar{W}_{j}}^{\prime \prime}\left(y_{j}^{n}, l_{\bar{S}_{k}}^{\prime}, \hat{l}_{\bar{D}_{j}, j}^{\prime \prime}, u_{D_{j}}^{n}\left(\hat{l}_{\bar{D}_{j}, j}^{\prime \prime}\right), u_{W_{j}}^{n}\left(\hat{l}_{\bar{D}_{j}, j}^{\prime \prime}, \tilde{l}_{\bar{W}_{j}}\right)\right.$ for all $\tilde{l}_{\bar{W}_{j}}$ such that $\tilde{l}_{i} \neq l_{i}^{\prime}$ for all $i \in$ $\left.\bar{S}_{k} \cap \bar{W}_{j}\right)$ at node $j \in[1: k-1]$ are chosen according to the following rule:

- Find the smallest $\hat{l}_{\bar{D}_{j}, j}^{\prime \prime} \in\left\{\tilde{l}_{\bar{D}_{j}}: \tilde{l}_{i} \neq l_{i}^{\prime}\right.$ for all $\left.i \in \bar{S}_{k} \cap \bar{D}_{j}\right\}$ such that

$$
\left(u_{D_{j} \cup B_{j}}^{n}\left(\hat{l}_{\bar{D}_{j}, j}^{\prime \prime}, \tilde{\tilde{l}}_{\bar{B}_{j}}^{\prime \prime}\right), y_{j}^{n}\right) \in \mathcal{T}_{\epsilon_{j}}^{(n)}
$$

for some $\tilde{\tilde{l}}_{\bar{B}_{j}}^{\prime \prime} \in\left\{\tilde{l}_{\bar{B}_{j}}: \tilde{l}_{i} \neq l_{i}^{\prime}\right.$ for all $\left.i \in \bar{S}_{k} \cap \bar{B}_{j}\right\}$. If there is no such index vector, let $\hat{l}_{\bar{D}_{j}, j}^{\prime \prime}$ be the smallest one in $\left\{\tilde{l}_{\bar{D}_{j}}: \tilde{l}_{i} \neq l_{i}^{\prime}\right.$ for all $\left.i \in \bar{S}_{k} \cap \bar{D}_{j}\right\}$.

- Find the smallest $l_{\bar{W}_{j}}^{\prime \prime} \in\left\{\tilde{l}_{\bar{W}_{j}}: \tilde{l}_{i} \neq l_{i}^{\prime}\right.$ for all $\left.i \in \bar{S}_{k} \cap \bar{W}_{j}\right\}$ such that

$$
\left(u_{D_{j}}^{n}\left(\hat{l}_{\bar{D}_{j}, j}^{\prime \prime}\right), u_{W_{j}}^{n}\left(\hat{l}_{\bar{D}_{j}, j}^{\prime \prime}, l_{\bar{W}_{j}}^{\prime \prime}\right), y_{j}^{n}\right) \in \mathcal{T}_{\epsilon_{j}^{\prime}}^{(n)} .
$$

If there is no such index vector, let $l_{\bar{W}_{j}}^{\prime \prime}$ be the smallest one in $\left\{\tilde{l}_{\bar{W}_{j}}: \tilde{l}_{i} \neq l_{i}^{\prime}\right.$ for all $\left.i \in \bar{S}_{k} \cap \bar{W}_{j}\right\}$. Note that $(a)$ follows because if $U_{D_{j} \cup B_{j}}^{n}(\cdot) \in \mathcal{A}_{j}\left(Y_{j}^{n}, l_{\bar{D}_{j} \cup \bar{B}_{j}}\right),\left(U_{\bar{D}_{j}}^{n}\left(l_{\bar{D}_{j}}\right), U_{W_{j}}^{n}\left(l_{\bar{D}_{j}}, \cdot\right)\right) \in \mathcal{B}_{j}\left(Y_{j}^{n}, l_{\bar{D}_{j} \cup \bar{W}_{j}}\right)$ for all $j \in[1: k-1]$ for some $l_{\bar{W}^{k-1}}$ such that $l_{i} \neq l_{i}^{\prime}$ for all $i \in \bar{S}_{k}$, then $\hat{L}_{\bar{D}_{j}, j}^{\prime \prime}=l_{\bar{D}_{j}}, L_{\bar{W}_{j}}^{\prime \prime}=l_{\bar{W}_{j}}$ for all $j \in[1: k-1]$, and hence $\tilde{Y}_{k}^{n}=Y_{k}^{n}$. 
By discarding some constraints, 477 is upper-bounded by

$$
\sum_{l_{\bar{S}_{k}}^{\prime}} P\left(\left(U_{D_{k} \cup B_{k}}^{n}\left(l_{\bar{S}_{k}}^{\prime}, L_{\bar{S}_{k}^{c}}^{\prime \prime}\right), \tilde{Y}_{k}^{n}\right) \in \mathcal{T}_{\epsilon_{k}}^{(n)}\right)
$$

Now, we can show that the joint distribution of $\left(U_{D_{k} \cup B_{k}}^{n}\left(l_{\bar{S}_{k}}^{\prime}, L_{\bar{S}_{k}^{c}}^{\prime \prime}\right), \tilde{Y}_{k}^{n}\right)$ is given as follows:

$$
P\left(U_{D_{k} \cup B_{k}}^{n}\left(l_{\bar{S}_{k}}^{\prime}, L_{\bar{S}_{k}^{c}}^{\prime \prime}\right)=u_{D_{k} \cup B_{k}}^{n}, \tilde{Y}_{k}^{n}=y_{k}^{n}\right)=p\left(u_{S_{k}^{c}}^{n}, y_{k}^{n}\right) \prod_{j \in S_{k}} \prod_{i=1}^{n} p\left(u_{j, i} \mid u_{A_{j}, i}\right),
$$

where $S_{k}$ is defined in (4).

Now, we can obtain the following upper bound:

$$
\begin{aligned}
& \sum_{l_{\bar{S}_{k}}^{\prime}} P\left(\left(U_{D_{k} \cup B_{k}}^{n}\left(l_{\bar{S}_{k}}^{\prime}, L_{\bar{S}_{k}^{c}}^{\prime \prime}\right), \tilde{Y}_{k}^{n}\right) \in \mathcal{T}_{\epsilon_{k}}^{(n)}\right) \\
& =2^{n \sum_{j \in \bar{S}_{k}} r_{j}} \sum_{\left(u_{S_{k}^{c}}^{n}, y_{k}^{n}\right) \in \mathcal{T}_{\epsilon_{k}}^{(n)}} \sum_{u_{S_{k}}^{n} \in \mathcal{T}_{\epsilon_{k}}^{(n)}\left(U_{S_{k}} \mid u_{S_{k}^{c}}^{n}, y_{k}^{n}\right)} p\left(u_{S_{k}^{c}}^{n}, y_{k}^{n}\right) \prod_{j \in S_{k}} \prod_{i=1}^{n} p\left(u_{j, i} \mid u_{A_{j}, i}\right) \\
& \leq 2^{n \sum_{j \in \bar{S}_{k}} r_{j}} \sum_{\left(u_{S_{k}^{c}}^{n}, y_{k}^{n}\right) \in \mathcal{T}_{\epsilon_{k}}^{(n)}} p\left(u_{S_{k}^{c}}^{n}, y_{k}^{n}\right) \cdot 2^{n\left(H\left(U_{S_{k}} \mid U_{S_{k}^{c}}, Y_{k}\right)+\delta\left(\epsilon_{k}\right)\right)} \cdot \prod_{j \in S_{k}} 2^{-n\left(H\left(U_{j} \mid U_{A_{j}}\right)-\delta\left(\epsilon_{k}\right)\right)} \\
& \leq 2^{n \sum_{j \in \bar{S}_{k}} r_{j}} \cdot 2^{-n\left(\sum_{j \in S_{k}} H\left(U_{j} \mid U_{A_{j}}\right)-H\left(U_{S_{k}} \mid U_{S_{k}^{c}}, Y_{k}\right)-(1+\nu) \delta\left(\epsilon_{k}\right)\right)} \\
& =2^{n \sum_{j \in \bar{S}_{k}} r_{j}} \cdot 2^{-n\left(\sum_{j \in S_{k}}\left(H\left(U_{j} \mid U_{A_{j}}\right)-H\left(U_{j} \mid U_{A_{j}}, U_{S_{k}[j]}, U_{S_{k}^{c}}, Y_{k}\right)\right)-(1+\nu) \delta\left(\epsilon_{k}\right)\right)} \\
& =2^{n \sum_{j \in \bar{S}_{k}} r_{j}} \cdot 2^{-n\left(\sum_{j \in S_{k}} I\left(U_{j} ; U_{S_{k}[j] \cup S_{k}^{c}}, Y_{k} \mid U_{A_{j}}\right)-(1+\nu) \delta\left(\epsilon_{k}\right)\right)},
\end{aligned}
$$

which tends to zero as $n$ tends to infinity if

$$
\sum_{j \in \bar{S}_{k}} r_{j}<\sum_{j \in S_{k}} I\left(U_{j} ; U_{S_{k}[j] \cup S_{k}^{c}}, Y_{k} \mid U_{A_{j}}\right)-(1+\nu) \delta\left(\epsilon_{k}\right) .
$$

Therefore, $P\left(\mathcal{E}_{k, 2} \cap \bigcap_{j=1}^{k-1}\left(\mathcal{E}_{j, 1} \cup \mathcal{E}_{j, 2} \cup \mathcal{E}_{j, 3}\right)^{c}\right)$ tends to zero as $n$ tends to infinity when 490) is satisfied for all $\bar{S}_{k} \subseteq \bar{D}_{k} \cup \bar{B}_{k}$ such that $\bar{S}_{k} \cap \bar{D}_{k} \neq \emptyset$.

\section{B. Bounding the third term in the summation in (8)}

The proof follows similar steps to the mutual covering lemma in [40]. For given $k \in[1: N]$, we have

$$
\begin{aligned}
& P\left(\mathcal{E}_{k, 3} \cap \mathcal{E}_{k, 1}^{c} \cap \mathcal{E}_{k, 2}^{c}\right) \\
& \leq P\left(\left(U_{D_{k}}^{n}\left(L_{\bar{D}_{k}}\right), Y_{k}^{n}\right) \in \mathcal{T}_{\epsilon_{k}}^{(n)},\left(U_{D_{k}}^{n}\left(L_{\bar{D}_{k}}\right), U_{W_{k}}^{n}\left(L_{\bar{D}_{k}}, l_{\bar{W}_{k}}\right), Y_{k}^{n}\right) \notin \mathcal{T}_{\epsilon_{k}^{\prime}}^{(n)} \text { for all } l_{\bar{W}_{k}}\right) \\
& =\sum_{l_{\bar{D}_{k}},\left(u_{D_{k}}^{n}, y_{k}^{n}\right) \in \mathcal{T}_{\epsilon_{k}}^{(n)}} P\left(L_{\bar{D}_{k}}=l_{\bar{D}_{k}}, U_{D_{k}}^{n}\left(l_{\bar{D}_{k}}\right)=u_{D_{k}}^{n}, Y_{k}^{n}=y_{k}^{n}\right) P\left(\left|\mathcal{L}_{k}\left(l_{\bar{D}_{k}}, u_{D_{k}}^{n}, y_{k}^{n}\right)\right|=0 \mid U_{D_{k}}^{n}\left(l_{\bar{D}_{k}}\right)=u_{D_{k}}^{n}\right)
\end{aligned}
$$


where

$$
\mathcal{L}_{k}\left(l_{\bar{D}_{k}}, u_{D_{k}}^{n}, y_{k}^{n}\right) \triangleq\left\{l_{\bar{W}_{k}}:\left(u_{D_{k}}^{n}, U_{W_{k}}^{n}\left(l_{\bar{D}_{k} \cup \bar{W}_{k}}\right), y_{k}^{n}\right) \in \mathcal{T}_{\epsilon_{k}^{\prime}}^{(n)}\right\} .
$$

Consider $l_{\bar{D}_{k}}$ and $\left(u_{D_{k}}^{n}, y_{k}^{n}\right) \in \mathcal{T}_{\epsilon_{k}}^{(n)}$. From the Chebyshev lemma, we have

$$
P\left(\left|\mathcal{L}_{k}\left(l_{\bar{D}_{k}}, u_{D_{k}}^{n}, y_{k}^{n}\right)\right|=0 \mid U_{D_{k}}^{n}\left(l_{\bar{D}_{k}}\right)=u_{D_{k}}^{n}\right) \leq \frac{\operatorname{Var}\left(\left|\mathcal{L}_{k}\left(l_{\bar{D}_{k}}, u_{D_{k}}^{n}, y_{k}^{n}\right)\right| \mid U_{D_{k}}^{n}\left(l_{\bar{D}_{k}}\right)=u_{D_{k}}^{n}\right)}{\left(\mathrm{E}\left[\left|\mathcal{L}_{k}\left(l_{\bar{D}_{k}}, u_{D_{k}}^{n}, y_{k}^{n}\right)\right| \mid U_{D_{k}}^{n}\left(l_{\bar{D}_{k}}\right)=u_{D_{k}}^{n}\right]\right)^{2}} .
$$

Now, define the indicator function

$$
I\left(l_{\bar{W}_{k}}\right)= \begin{cases}1 & \text { if }\left(u_{D_{k}}^{n}, U_{W_{k}}^{n}\left(l_{\bar{D}_{k} \cup \bar{W}_{k}}\right), y_{k}^{n}\right) \in \mathcal{T}_{\epsilon_{k}^{\prime}}^{(n)} \\ 0 & \text { otherwise }\end{cases}
$$

for each $l_{\bar{W}_{k}}$. Note that $\left|\mathcal{L}_{k}\left(l_{\bar{D}_{k}}, u_{D_{k}}^{n}, y_{k}^{n}\right)\right|=\sum_{l_{\bar{W}_{k}}} I\left(l_{\bar{W}_{k}}\right)$.

Due to the symmetry of the codebook generation, for any $l_{\bar{W}_{k}}, l_{\bar{W}_{k}}^{\prime}, \tilde{l}_{\bar{W}_{k}}, \tilde{l}_{\bar{W}_{k}}^{\prime}$, and $\bar{T}_{k} \subseteq \bar{W}_{k}$ such that $l_{\bar{T}_{k}}=l_{\bar{T}_{k}}^{\prime}, \tilde{l}_{\bar{T}_{k}}=\tilde{l}_{\bar{T}_{k}}^{\prime}$ and $l_{i} \neq l_{i}^{\prime}, \tilde{l}_{i} \neq \tilde{l}_{i}^{\prime}$ for all $i \notin \bar{T}_{k}$, we have $\mathrm{E}\left[I\left(l_{\bar{W}_{k}}\right) I\left(l_{\bar{W}_{k}}^{\prime}\right) \mid U_{D_{k}}^{n}\left(l_{\bar{D}_{k}}\right)=\right.$ $\left.u_{D_{k}}^{n}\right]=\mathrm{E}\left[I\left(\tilde{l}_{\bar{W}_{k}}\right) I\left(\tilde{l}_{\bar{W}_{k}}^{\prime}\right) \mid U_{D_{k}}^{n}\left(l_{\bar{D}_{k}}\right)=u_{D_{k}}^{n}\right]$. Let $p_{\bar{T}_{k}}$ for $\bar{T}_{k} \subseteq \bar{W}_{k}$ denote $\mathrm{E}\left[I\left(l_{\bar{W}_{k}}\right) I\left(l_{\bar{W}_{k}}^{\prime}\right) \mid l_{\bar{T}_{k}}=l_{\bar{T}_{k}}^{\prime}, l_{i} \neq\right.$ $l_{i}^{\prime}$ for all $\left.i \notin \bar{T}_{k}, U_{D_{k}}^{n}\left(l_{\bar{D}_{k}}\right)=u_{D_{k}}^{n}\right]$.

Then, we have

$$
\begin{aligned}
\mathrm{E}\left[\left|\mathcal{L}_{k}\left(l_{\bar{D}_{k}}, u_{D_{k}}^{n}, y_{k}^{n}\right)\right| \mid U_{D_{k}}^{n}\left(l_{\bar{D}_{k}}\right)=u_{D_{k}}^{n}\right] & =\sum_{l_{\bar{W}_{k}}} \mathrm{E}\left[I\left(l_{\bar{W}_{k}}\right) \mid U_{D_{k}}^{n}\left(l_{\bar{D}_{k}}\right)=u_{D_{k}}^{n}\right] \\
& =\sum_{l_{\bar{W}_{k}}} \mathrm{E}\left[I^{2}\left(l_{\bar{W}_{k}}\right) \mid U_{D_{k}}^{n}\left(l_{\bar{D}_{k}}\right)=u_{D_{k}}^{n}\right] \\
& =2^{n \sum_{i \in \bar{W}_{k}}{ }^{r_{i}} p_{\bar{W}_{k}}}
\end{aligned}
$$

and

$$
\begin{aligned}
\mathrm{E}\left[\left|\mathcal{L}_{k}\left(l_{\bar{D}_{k}}, u_{D_{k}}^{n}, y_{k}^{n}\right)\right|^{2} \mid U_{D_{k}}^{n}\left(l_{\bar{D}_{k}}\right)=u_{D_{k}}^{n}\right] & =\sum_{l_{\bar{W}_{k}}} \sum_{\bar{T}_{k} \subseteq \bar{W}_{k}} \sum_{l_{\bar{T}_{k}}^{\prime}: l_{i}^{\prime} \neq l_{i}, \forall i \in \bar{T}_{k}^{c}} \mathrm{E}\left[I\left(l_{\bar{W}_{k}}\right) I\left(l_{\bar{T}_{k}}, l_{\bar{T}_{k}^{c}}^{\prime}\right) \mid U_{D_{k}}^{n}\left(l_{\bar{D}_{k}}\right)=u_{D_{k}}^{n}\right] \\
& =\sum_{\bar{T}_{k} \subseteq \bar{W}_{k}} \sum_{l_{\bar{W}_{k}}} \sum_{l_{\bar{T}_{k}}^{\prime}: l_{i}^{\prime} \neq l_{i}, \forall i \in \bar{T}_{k}^{c}} \mathrm{E}\left[I\left(l_{\bar{W}_{k}}\right) I\left(l_{\bar{T}_{k}}, l_{\bar{T}_{k}^{c}}^{\prime}\right) \mid U_{D_{k}}^{n}\left(l_{\bar{D}_{k}}\right)=u_{D_{k}}^{n}\right] \\
& \leq \sum_{\bar{T}_{k} \subseteq \bar{W}_{k}} 2^{n\left(\sum_{i \in \bar{T}_{k}} r_{i}+2 \sum_{i \in \bar{T}_{k}^{c}} r_{i}\right)} p_{\bar{T}_{k}} .
\end{aligned}
$$

Since $p_{\emptyset}=p_{\bar{W}_{k}}^{2}$, we have

$$
\begin{aligned}
& \operatorname{Var}\left(\left|\mathcal{L}_{k}\left(l_{\bar{D}_{k}}, u_{D_{k}}^{n}, y_{k}^{n}\right)\right| \mid U_{D_{k}}^{n}\left(l_{\bar{D}_{k}}\right)=u_{D_{k}}^{n}\right) \\
& =\mathrm{E}\left[\left|\mathcal{L}_{k}\left(l_{\bar{D}_{k}}, u_{D_{k}}^{n}, y_{k}^{n}\right)\right|^{2} \mid U_{D_{k}}^{n}\left(l_{\bar{D}_{k}}\right)=u_{D_{k}}^{n}\right]-\mathrm{E}^{2}\left[\left|\mathcal{L}_{k}\left(l_{\bar{D}_{k}}, u_{D_{k}}^{n}, y_{k}^{n}\right)\right| \mid U_{D_{k}}^{n}\left(l_{\bar{D}_{k}}\right)=u_{D_{k}}^{n}\right] \\
& \leq \sum_{\bar{T}_{k} \subseteq \bar{W}_{k}, \bar{T}_{k} \neq \emptyset} 2^{n\left(\sum_{i \in \bar{T}_{k}} r_{i}+2 \sum_{i \in \bar{T}_{k}^{c}} r_{i}\right)} p_{\bar{T}_{k}} .
\end{aligned}
$$


Now, for $\bar{T}_{k} \subseteq \bar{W}_{k}$ such that $\bar{T}_{k} \neq \emptyset$, we have

$$
\begin{gathered}
p_{\bar{T}_{k}}=P\left(\left(u_{D_{k}}^{n}, U_{W_{k}}^{n}\left(l_{\bar{D}_{k} \cup \bar{W}_{k}}\right), y_{k}^{n}\right) \in \mathcal{T}_{\epsilon_{k}^{\prime}}^{(n)},\left(u_{D_{k}}^{n}, U_{W_{k}}^{n}\left(l_{\bar{D}_{k}}, l_{\bar{W}_{k}}^{\prime}\right), y_{k}^{n}\right) \in \mathcal{T}_{\epsilon_{k}^{\prime}}^{(n)} \mid l_{\bar{T}_{k}}=l_{\bar{T}_{k}}^{\prime},\right. \\
\left.l_{i} \neq l_{i}^{\prime} \text { for all } i \notin \bar{T}_{k}, U_{D_{k}}^{n}\left(l_{\bar{D}_{k}}\right)=u_{D_{k}}^{n}\right) \\
\leq 2^{-n\left(\sum_{j \in T_{k}} I\left(U_{j} ; U_{T_{k}[j] \cup D_{k}}, Y_{k} \mid U_{A_{j}}\right)+2 \sum_{j \in T_{k}^{c}} I\left(U_{j} ; U_{T_{k} \cup T_{k}^{c}[j] \cup D_{k}}, Y_{k} \mid U_{A_{j}}\right)-2(1+\nu) \delta\left(\epsilon_{k}^{\prime}\right)\right)}
\end{gathered}
$$

by the joint typicality lemma [41], where $T_{k}$ is defined in (5). Similarly, for $\bar{T}_{k} \subseteq \bar{W}_{k}$ such that $\bar{T}_{k} \neq \emptyset$, we have

$$
p_{\bar{W}_{k}} \geq 2^{-n\left(\sum_{j \in T_{k}} I\left(U_{j} ; U_{T_{k}[j] \cup D_{k}}, Y_{k} \mid U_{A_{j}}\right)+\sum_{j \in T_{k}^{c}} I\left(U_{j} ; U_{T_{k} \cup T_{k}^{c}[j] \cup D_{k}}, Y_{k} \mid U_{A_{j}}\right)+(1+\nu) \delta\left(\epsilon_{k}^{\prime}\right)\right)} .
$$

By substituting the above bounds into (50) and (51), we obtain

$$
\frac{\operatorname{Var}\left(\left|\mathcal{L}_{k}\left(l_{\bar{D}_{k}}, u_{D_{k}}^{n}, y_{k}^{n}\right)\right| \mid U_{D_{k}}^{n}\left(l_{\bar{D}_{k}}\right)=u_{D_{k}}^{n}\right)}{\left(\mathrm{E}\left[\left|\mathcal{L}_{k}\left(l_{\bar{D}_{k}}, u_{D_{k}}^{n}, y_{k}^{n}\right)\right| \mid U_{D_{k}}^{n}\left(l_{\bar{D}_{k}}\right)=u_{D_{k}}^{n}\right]\right)^{2}} \leq \sum_{\bar{T}_{k} \subseteq \bar{W}_{k}, \bar{T}_{k} \neq \emptyset} 2^{-n\left(\sum_{j \in \bar{T}_{k}} r_{j}-\sum_{j \in T_{k}} I\left(U_{j} ; U_{T_{k}[j] \cup D_{k}}, Y_{k} \mid U_{A_{j}}\right)-4(1+\nu) \delta\left(\epsilon_{k}^{\prime}\right)\right)} .
$$

Therefore, $P\left(\left|\mathcal{L}_{k}\left(l_{\bar{D}_{k}}, u_{D_{k}}^{n}, y_{k}^{n}\right)\right|=0 \mid U_{D_{k}}^{n}\left(l_{\bar{D}_{k}}\right)=u_{D_{k}}^{n}\right)$ and thus $P\left(\mathcal{E}_{k, 3} \cap \mathcal{E}_{k, 1}^{c} \cap \mathcal{E}_{k, 2}^{c}\right)$ tend to zero as $n$ tends to infinity if

$$
\sum_{j \in \bar{T}_{k}} r_{j}>\sum_{j \in T_{k}} I\left(U_{j} ; U_{T_{k}[j] \cup D_{k}}, Y_{k} \mid U_{A_{j}}\right)+4(1+\nu) \delta\left(\epsilon_{k}^{\prime}\right)
$$

for all $\bar{T}_{k} \subseteq \bar{W}_{k}$ such that $\bar{T}_{k} \neq \emptyset$.

\section{Bounding the fourth term in the summation in (8)}

For given $k \in[1: N]$, we have

$$
\begin{aligned}
& P\left(\mathcal{E}_{k, 4} \cap \mathcal{E}_{k, 1}^{c} \cap \mathcal{E}_{k, 2}^{c}\right) \\
& \leq P\left(\left(U_{W^{k-1}}^{n}\left(L_{\bar{W}^{k-1}}\right), Y_{[1: k]}^{n}\right) \in \mathcal{T}_{\epsilon_{k}}^{(n)},\left(U_{W^{k}}^{n}\left(L_{\bar{W}^{k}}\right), Y_{[1: k]}^{n}\right) \notin \mathcal{T}_{\epsilon_{k}^{\prime \prime}}^{(n)}, \hat{L}_{\bar{D}_{k}, k}=L_{\bar{D}_{k}}\right) \\
& \leq P\left(\left(U_{W^{k-1}}^{n}\left(\hat{L}_{\bar{D}_{k}, k}, L_{\bar{W}^{k-1} \backslash \bar{D}_{k}}\right), Y_{[1: k]}^{n}\right) \in \mathcal{T}_{\epsilon_{k}}^{(n)},\right. \\
& \left.\quad\left(U_{W^{k-1}}^{n}\left(\hat{L}_{\bar{D}_{k}, k}, L_{\bar{W}^{k-1} \backslash \bar{D}_{k}}\right), Y_{[1: k]}^{n}, U_{W_{k}}^{n}\left(\hat{L}_{\bar{D}_{k}, k}, L_{\bar{W}_{k}}\right)\right) \notin \mathcal{T}_{\epsilon_{k}^{\prime \prime}}^{(n)}\right) \\
& \quad \sum_{l_{\bar{D}_{k}},\left(u_{W^{k-1}}^{n}, y_{[1: k]}^{n}\right) \in \mathcal{T}_{\epsilon_{k}}^{(n)}} P\left(\hat{L}_{\bar{D}_{k}, k}=l_{\bar{D}_{k}}, U_{W^{k-1}}^{n}\left(l_{\bar{D}_{k}}, L_{\bar{W}^{k-1} \backslash \bar{D}_{k}}\right)=u_{W^{k-1}}^{n}, Y_{[1: k]}^{n}=y_{[1: k]}^{n}\right) \\
& \quad \cdot P\left(\left(u_{W^{k-1}}^{n}, y_{[1: k]}^{n}, U_{W_{k}}^{n}\left(l_{\bar{D}_{k}}, L_{\bar{W}_{k}}\right)\right) \notin \mathcal{T}_{\epsilon_{k}^{\prime \prime}}^{(n)} \mid \hat{L}_{\bar{D}_{k}, k}=l_{\bar{D}_{k}}, U_{W^{k-1}}^{n}\left(l_{\bar{D}_{k}}, L_{\bar{W}^{k-1} \backslash \bar{D}_{k}}\right)=u_{W^{k-1}}^{n}, Y_{[1: k]}^{n}=y_{[1: k]}^{n}\right)
\end{aligned}
$$

We use the following modified Markov lemma to bound the above, which can be proved from the proof of the Markov lemma in [28], [41] with some minor modification. 
Lemma 3: Consider random variables $X, Y, Z, A$ such that $X \rightarrow Y \rightarrow Z$ form a Markov chain. Let $\left(x^{n}, y^{n}\right) \in \mathcal{T}_{\epsilon}^{(n)}$ and $a \in \mathcal{A}$. Suppose that $P\left(Z^{n}=z^{n} \mid X^{n}=x^{n}, Y^{n}=y^{n}, A=a\right)=P\left(Z^{n}=z^{n} \mid Y^{n}=\right.$ $\left.y^{n}, A=a\right)$, where $P\left(Z^{n}=z^{n} \mid Y^{n}=y^{n}, A=a\right)$ satisfies the following conditions for $\epsilon^{\prime}>\epsilon$ :

1) $\lim _{n \rightarrow \infty} P\left(\left(y^{n}, Z^{n}\right) \in \mathcal{T}_{\epsilon^{\prime}}^{(n)} \mid Y^{n}=y^{n}, A=a\right)=1$.

2) For every $z^{n} \in \mathcal{T}_{\epsilon^{\prime}}^{(n)}\left(Z \mid y^{n}\right)$ and $n$ sufficiently large,

$$
P\left(Z^{n}=z^{n} \mid Y^{n}=y^{n}, A=a\right) \leq 2^{-n\left(H(Z \mid Y)-\delta\left(\epsilon^{\prime}\right)\right)} .
$$

Then, for sufficiently small $\epsilon$ and $\epsilon^{\prime}$ such that $\epsilon<\epsilon^{\prime}<\epsilon^{\prime \prime}$,

$$
\lim _{n \rightarrow \infty} P\left(\left(x^{n}, y^{n}, Z^{n}\right) \in \mathcal{T}_{\epsilon^{\prime \prime}}^{(n)} \mid X^{n}=x^{n}, Y^{n}=y^{n}, A=a\right)=1 .
$$

Fix $l_{\bar{D}_{k}}$ and $\left(u_{W^{k-1}}^{n}, y_{[1: k]}^{n}\right) \in \mathcal{T}_{\epsilon_{k}}^{(n)}$. We use Lemma 3 to show

$$
\begin{aligned}
& \lim _{n \rightarrow \infty} P\left(\left(u_{W^{k-1}}^{n}, y_{[1: k]}^{n}, U_{W_{k}}^{n}\left(l_{\bar{D}_{k}}, L_{\bar{W}_{k}}\right)\right) \in \mathcal{T}_{\epsilon_{k}^{\prime \prime}}^{(n)} \mid \hat{L}_{\bar{D}_{k}, k}=l_{\bar{D}_{k}},\right. \\
& \left.\quad U_{W^{k-1}}^{n}\left(l_{\bar{D}_{k}}, L_{\bar{W}^{k-1} \backslash \bar{D}_{k}}\right)=u_{W^{k-1}}^{n}, Y_{[1: k]}^{n}=y_{[1: k]}^{n}\right)=1 .
\end{aligned}
$$

Note that $\left(U_{W^{k-1} \backslash D_{k}}, Y_{[1: k-1]}\right)-\left(U_{D_{k}}, Y_{k}\right)-U_{W_{k}}$ form a Markov chain and

$$
\begin{aligned}
& P\left(U_{W_{k}}^{n}\left(l_{\bar{D}_{k}}, L_{\bar{W}_{k}}\right)=u_{W_{k}}^{n} \mid \hat{L}_{\bar{D}_{k}, k}=l_{\bar{D}_{k}}, U_{W^{k-1}}^{n}\left(l_{\bar{D}_{k}}, L_{\bar{W}^{k-1} \backslash \bar{D}_{k}}\right)=u_{W^{k-1}}^{n}, Y_{[1: k]}^{n}=y_{[1: k]}^{n}\right) \\
& =P\left(U_{W_{k}}^{n}\left(l_{\bar{D}_{k}}, L_{\bar{W}_{k}}\right)=u_{W_{k}}^{n} \mid \hat{L}_{\bar{D}_{k}, k}=l_{\bar{D}_{k}}, U_{D_{k}}^{n}\left(l_{\bar{D}_{k}}\right)=u_{D_{k}}^{n}, Y_{k}^{n}=y_{k}^{n}\right) .
\end{aligned}
$$

The first condition in Lemma $\underline{3}$ is safisfied if (52) is satisfied for all $\bar{T}_{k} \subseteq \bar{W}_{k}$ such that $\bar{T}_{k} \neq \emptyset$ since

$$
\begin{aligned}
& P\left(\left(u_{D_{k}}^{n}, y_{k}^{n}, U_{W_{k}}^{n}\left(l_{\bar{D}_{k}}, L_{\bar{W}_{k}}\right)\right) \in \mathcal{T}_{\epsilon_{k}^{\prime}}^{(n)} \mid \hat{L}_{\bar{D}_{k}, k}=l_{\bar{D}_{k}}, U_{D_{k}}^{n}\left(l_{\bar{D}_{k}}\right)=u_{D_{k}}^{n}, Y_{k}^{n}=y_{k}^{n}\right) \\
& =1-P\left(\left(u_{D_{k}}^{n}, y_{k}^{n}, U_{W_{k}}^{n}\left(l_{\bar{D}_{k}}, l_{\bar{W}_{k}}\right)\right) \notin \mathcal{T}_{\epsilon_{k}^{\prime}}^{(n)} \text { for all } l_{\bar{W}_{k}} \mid \hat{L}_{\bar{D}_{k}, k}=l_{\bar{D}_{k}}, U_{D_{k}}^{n}\left(l_{\bar{D}_{k}}\right)=u_{D_{k}}^{n}, Y_{k}^{n}=y_{k}^{n}\right) \\
& =1-P\left(\left(u_{D_{k}}^{n}, y_{k}^{n}, U_{W_{k}}^{n}\left(l_{\bar{D}_{k}}, l_{\bar{W}_{k}}\right)\right) \notin \mathcal{T}_{\epsilon_{k}^{\prime}}^{(n)} \text { for all } l_{\bar{W}_{k}} \mid U_{D_{k}}^{n}\left(l_{\bar{D}_{k}}\right)=u_{D_{k}}^{n}\right)
\end{aligned}
$$

and we have showed in the analysis of the third error event

$$
\lim _{n \rightarrow \infty} P\left(\left(u_{D_{k}}^{n}, y_{k}^{n}, U_{W_{k}}^{n}\left(l_{\bar{D}_{k}}, l_{\bar{W}_{k}}\right)\right) \notin \mathcal{T}_{\epsilon_{k}^{\prime}}^{(n)} \text { for all } l_{\bar{W}_{k}} \mid U_{D_{k}}^{n}\left(l_{\bar{D}_{k}}\right)=u_{D_{k}}^{n}\right)=0
$$

under the aforementioned condition. 
Now, let us show that the second condition in Lemma 3 is satisfied. For every $u_{W_{k}}^{n} \in \mathcal{T}_{\epsilon_{k}^{\prime}}^{(n)}\left(U_{W_{k}} \mid u_{D_{k}}^{n}, y_{k}^{n}\right)$,

$$
\begin{gathered}
P\left(U_{W_{k}}^{n}\left(l_{\bar{D}_{k}}, L_{\bar{W}_{k}}\right)=u_{W_{k}}^{n} \mid \hat{L}_{\bar{D}_{k}, k}=l_{\bar{D}_{k}}, U_{D_{k}}^{n}\left(l_{\bar{D}_{k}}\right)=u_{D_{k}}^{n}, Y_{k}^{n}=y_{k}^{n}\right) \\
=P\left(U_{W_{k}}^{n}\left(l_{\bar{D}_{k}}, L_{\bar{W}_{k}}\right)=u_{W_{k}}^{n}, U_{W_{k}}^{n}\left(l_{\bar{D}_{k}}, L_{\bar{W}_{k}}\right) \in \mathcal{T}_{\epsilon_{k}^{\prime}}^{(n)}\left(U_{W_{k}} \mid u_{D_{k}}^{n}, y_{k}^{n}\right) \mid \hat{L}_{\bar{D}_{k}, k}=l_{\bar{D}_{k}}, U_{D_{k}}^{n}\left(l_{\bar{D}_{k}}\right)=u_{D_{k}}^{n}, Y_{k}^{n}=y_{k}^{n}\right) \\
\leq P\left(U_{W_{k}}^{n}\left(l_{\bar{D}_{k}}, L_{\bar{W}_{k}}\right)=u_{W_{k}}^{n} \mid \hat{L}_{\bar{D}_{k}, k}=l_{\bar{D}_{k}}, U_{D_{k}}^{n}\left(l_{\bar{D}_{k}}\right)=u_{D_{k}}^{n}, Y_{k}^{n}=y_{k}^{n}, U_{W_{k}}^{n}\left(l_{\bar{D}_{k}}, L_{\bar{W}_{k}}\right) \in \mathcal{T}_{\epsilon_{k}^{\prime}}^{(n)}\left(U_{W_{k}} \mid u_{D_{k}}^{n}, y_{k}^{n}\right)\right) \\
=\sum_{l_{\bar{W}_{k}}} P\left(L_{\bar{W}_{k}}=l_{\bar{W}_{k}} \mid \hat{L}_{\bar{D}_{k}, k}=l_{\bar{D}_{k}}, U_{D_{k}}^{n}\left(l_{\bar{D}_{k}}\right)=u_{D_{k}}^{n}, Y_{k}^{n}=y_{k}^{n}, U_{W_{k}}^{n}\left(l_{\bar{D}_{k}}, L_{\bar{W}_{k}}\right) \in \mathcal{T}_{\epsilon_{k}^{\prime}}^{(n)}\left(U_{W_{k}} \mid u_{D_{k}}^{n}, y_{k}^{n}\right)\right) \\
\cdot P\left(U_{W_{k}}^{n}\left(l_{\bar{D}_{k} \cup \bar{W}_{k}}\right)=u_{W_{k}}^{n} \mid \hat{L}_{\bar{D}_{k}, k}=l_{\bar{D}_{k}}, L_{\bar{W}_{k}}=l_{\bar{W}_{k}}, U_{D_{k}}^{n}\left(l_{\bar{D}_{k}}\right)=u_{D_{k}}^{n}, Y_{k}^{n}=y_{k}^{n},\right. \\
\left.U_{W_{k}}^{n}\left(l_{\bar{D}_{k} \cup \bar{W}_{k}}\right) \in \mathcal{T}_{\epsilon_{k}^{\prime}}^{(n)}\left(U_{W_{k}} \mid u_{D_{k}}^{n}, y_{k}^{n}\right)\right) .
\end{gathered}
$$

For given $l_{\bar{W}_{k}}$, we have

$$
\begin{aligned}
& P\left(U_{W_{k}}^{n}\left(l_{\bar{D}_{k} \cup \bar{W}_{k}}\right)=u_{W_{k}}^{n} \mid \hat{L}_{\bar{D}_{k}, k}=l_{\bar{D}_{k}}, L_{\bar{W}_{k}}=l_{\bar{W}_{k}}, U_{D_{k}}^{n}\left(l_{\bar{D}_{k}}\right)=u_{D_{k}}^{n}, Y_{k}^{n}=y_{k}^{n},\right. \\
& \left.\quad U_{W_{k}}^{n}\left(l_{\bar{D}_{k} \cup \bar{W}_{k}}\right) \in \mathcal{T}_{\epsilon_{k}^{\prime}}^{(n)}\left(U_{W_{k}} \mid u_{D_{k}}^{n}, y_{k}^{n}\right)\right) \\
& \stackrel{(a)}{=} P\left(U_{W_{k}}^{n}\left(l_{\bar{D}_{k} \cup \bar{W}_{k}}\right)=u_{W_{k}}^{n} \mid U_{D_{k}}^{n}\left(l_{\bar{D}_{k}}\right)=u_{D_{k}}^{n}, U_{W_{k}}^{n}\left(l_{\bar{D}_{k} \cup \bar{W}_{k}}\right) \in \mathcal{T}_{\epsilon_{k}^{\prime}}^{(n)}\left(U_{W_{k}} \mid u_{D_{k}}^{n}, y_{k}^{n}\right)\right) \\
& =\frac{P\left(U_{W_{k}}^{n}\left(l_{\bar{D}_{k} \cup \bar{W}_{k}}\right)=u_{W_{k}}^{n} \mid U_{D_{k}}^{n}\left(l_{\bar{D}_{k}}\right)=u_{D_{k}}^{n}\right)}{P\left(U_{W_{k}}^{n}\left(l_{\bar{D}_{k} \cup \bar{W}_{k}}\right) \in \mathcal{T}_{\epsilon_{k}^{\prime}}^{(n)}\left(U_{W_{k}} \mid u_{D_{k}}^{n}, y_{k}^{n}\right) \mid U_{D_{k}}^{n}\left(l_{\bar{D}_{k}}\right)=u_{D_{k}}^{n}\right)} \\
& \leq 2^{-n\left(H\left(U_{W_{k}} \mid U_{D_{k}}, Y_{k}\right)-\delta\left(\epsilon_{k}^{\prime}\right)\right)},
\end{aligned}
$$

where $(a)$ is because

$$
\begin{aligned}
& P\left(U_{W_{k}}^{n}\left(l_{\bar{D}_{k} \cup \bar{W}_{k}}\right)=u_{W_{k}}^{n}, \hat{L}_{\bar{D}_{k}, k}=l_{\bar{D}_{k}}, L_{\bar{W}_{k}}=l_{\bar{W}_{k}}, U_{D_{k}}^{n}\left(l_{\bar{D}_{k}}\right)=u_{D_{k}}^{n}, Y_{k}^{n}=y_{k}^{n},\right. \\
& \left.\quad U_{W_{k}}^{n}\left(l_{\bar{D}_{k} \cup \bar{W}_{k}}\right) \in \mathcal{T}_{\epsilon_{k}^{\prime}}^{(n)}\left(U_{W_{k}} \mid u_{D_{k}}^{n}, y_{k}^{n}\right)\right) \\
& =P\left(\hat{L}_{\bar{D}_{k}, k}=l_{\bar{D}_{k}}, U_{D_{k}}^{n}\left(l_{\bar{D}_{k}}\right)=u_{D_{k}}^{n}, Y_{k}^{n}=y_{k}^{n}\right) \\
& \quad \times P\left(U_{W_{k}}^{n}\left(l_{\bar{D}_{k} \cup \bar{W}_{k}}\right)=u_{W_{k}}^{n}, U_{W_{k}}^{n}\left(l_{\bar{D}_{k} \cup \bar{W}_{k}}\right) \in \mathcal{T}_{\epsilon_{k}^{\prime}}^{(n)}\left(U_{W_{k}} \mid u_{D_{k}}^{n}, y_{k}^{n}\right) \mid U_{D_{k}}^{n}\left(l_{\bar{D}_{k}}\right)=u_{D_{k}}^{n}\right) \\
& \quad \times P\left(L_{\bar{W}_{k}}=l_{\bar{W}_{k}} \mid \hat{L}_{\bar{D}_{k}, k}=l_{\bar{D}_{k}}, U_{D_{k}}^{n}\left(l_{\bar{D}_{k}}\right)=u_{D_{k}}^{n}, Y_{k}^{n}=y_{k}^{n}, U_{W_{k}}^{n}\left(l_{\bar{D}_{k} \cup \bar{W}_{k}}\right) \in \mathcal{T}_{\epsilon_{k}^{\prime}}^{(n)}\left(U_{W_{k}} \mid u_{D_{k}}^{n}, y_{k}^{n}\right)\right) \\
& =P\left(\hat{L}_{\bar{D}_{k}, k}=l_{\bar{D}_{k}}, U_{D_{k}}^{n}\left(l_{\bar{D}_{k}}\right)=u_{D_{k}}^{n}, Y_{k}^{n}=y_{k}^{n}, U_{W_{k}}^{n}\left(l_{\bar{D}_{k} \cup \bar{W}_{k}}\right) \in \mathcal{T}_{\epsilon_{k}^{\prime}}^{(n)}\left(U_{W_{k}} \mid u_{D_{k}}^{n}, y_{k}^{n}\right), L_{\bar{W}_{k}}=l_{\bar{W}_{k}}\right) \\
& \quad \times P\left(U_{W_{k}}^{n}\left(l_{\bar{D}_{k} \cup \bar{W}_{k}}\right)=u_{W_{k}}^{n} \mid U_{D_{k}}^{n}\left(l_{\bar{D}_{k}}\right)=u_{D_{k}}^{n}, U_{W_{k}}^{n}\left(l_{\bar{D}_{k} \cup \bar{W}_{k}}\right) \in \mathcal{T}_{\epsilon_{k}^{\prime}}^{(n)}\left(U_{W_{k}} \mid u_{D_{k}}^{n}, y_{k}^{n}\right)\right) .
\end{aligned}
$$

Hence, the second condition in Lemma 3 is satisfied.

Now, from Lemma 3, (53) holds and hence $P\left(\mathcal{E}_{k, 4} \cap \mathcal{E}_{k, 1}^{c} \cap \mathcal{E}_{k, 2}^{c}\right)$ tends to zero as $n$ tends to infinity for sufficiently small $\epsilon_{k}$ and $\epsilon_{k}^{\prime}$ if (52) is satisfied for all $\bar{T}_{k} \subseteq \bar{W}_{k}$ such that $\bar{T}_{k} \neq \emptyset$. 


\section{Special cases}

In this appendix, we present more examples of previous results that can be obtained as simple corollaries of our theorem.

1) Channels with action-dependent states [21]:

- ADMN: $N=3, H\left(Y_{1}\right)=R, Y_{2}=\left(X_{1}, Y_{1}, S\right), p\left(s \mid x_{1}, y_{1}\right)=p\left(s \mid x_{1}\right), p\left(y_{3} \mid y_{[1: 2]}, x_{[1: 2]}\right)=p\left(y_{3} \mid x_{2}, s\right)$.

- Target distribution: $p^{*}$ such that $X_{3}=Y_{1}$.

- Choice of $\omega^{\prime} \in \Omega^{\prime}$ in Corollary $11 \mu=2, U_{1}=\left(Y_{1}, X_{1}\right), W_{1}=\{1\}, W_{2}=\{2\}, D_{2}=\{1\}, D_{3}=$ $\{1\}, B_{3}=\{2\}, A_{2}=\{1\}, p\left(x_{1} \mid y_{1}\right)=p\left(x_{1}\right), p\left(u_{2} \mid y_{2}, u_{1}\right)=p\left(u_{2} \mid s, x_{1}\right), x_{2}\left(y_{2}, u_{1}, u_{2}\right)=x_{2}\left(s, u_{2}\right)$, $x_{3}\left(y_{3}, u_{1}\right)=y_{1}$.

2) Marton's inner bound with common message [53]:

- ADMN: $N=3, Y_{1}=\left(M_{1}, M_{2}, M_{3}\right), H\left(M_{k}\right)=R_{k}$ for $k \in[1: 3], p\left(y_{1}\right)=p\left(m_{1}\right) p\left(m_{2}\right) p\left(m_{3}\right)$, $p\left(y_{2} \mid y_{1}, x_{1}\right)=p\left(y_{2} \mid x_{1}\right), p\left(y_{3} \mid y_{[1: 2]}, x_{[1: 2]}\right)=p\left(y_{3} \mid x_{1}, y_{2}\right)$.

- Target distribution: $p^{*}$ such that $X_{2}=\left(M_{1}, M_{2}\right), X_{3}=\left(M_{1}, M_{3}\right)$.

- Choice of $\omega^{\prime} \in \Omega^{\prime}$ in Corollary $11 \mu=3, U_{j}=\left(M_{j}, V_{j}\right)$ for $j \in[1: 3], W_{1}=\{1,2,3\}, D_{2}=$ $\{1,2\}, D_{3}=\{1,3\}, A_{2}=\{1\}, A_{3}=\{1\}, p\left(v_{[1: 3]} \mid y_{1}\right)=p\left(v_{[1: 3]}\right), x_{1}\left(u_{[1: 3]}, y_{1}\right)=x_{1}\left(v_{[1: 3]}\right)$, $x_{2}\left(u_{[1: 2]}, y_{2}\right)=\left(m_{1}, m_{2}\right), x_{3}\left(u_{\{1,3\}}, y_{3}\right)=\left(m_{1}, m_{3}\right)$.

3) Three-receiver multilevel broadcast channel [38]:

- $\mathrm{ADMN}: N=4, Y_{1}=\left(M_{0}, M_{10}, M_{11}\right), H\left(M_{0}\right)=R_{0}, H\left(M_{10}\right)=R_{10}, H\left(M_{11}\right)=R_{11}, R_{1}=$ $R_{10}+R_{11}, p\left(y_{1}\right)=p\left(m_{0}\right) p\left(m_{10}\right) p\left(m_{11}\right), p\left(y_{2} \mid y_{1}, x_{1}\right)=p\left(y_{2} \mid x_{1}\right), p\left(y_{3} \mid y_{[1: 2]}, x_{[1: 2]}\right)=p\left(y_{3} \mid y_{2}\right)$, $p\left(y_{4} \mid y_{[1: 3]}, x_{[1: 3]}\right)=p\left(y_{4} \mid y_{2}, x_{1}\right)$.

- Target distribution: $p^{*}$ such that $X_{2}=\left(M_{0}, M_{10}, M_{11}\right), X_{3}=M_{0}, X_{4}=M_{0}$.

- Choice of $\omega^{\prime} \in \Omega^{\prime}$ in Corollary $1 \mu=3, U_{1}=\left(M_{0}, V_{1}\right), U_{2}=\left(M_{10}, V_{2}\right), U_{3}=\left(M_{11}, X_{1}\right)$, $W_{1}=\{1,2,3\}, D_{2}=\{1,2,3\}, D_{3}=\{1\}, D_{4}=\{1\}, B_{4}=\{2\}, A_{2}=\{1\}, A_{3}=\{1,2\}$, $p\left(v_{[1: 2]}, x_{1} \mid y_{1}\right)=p\left(v_{[1: 2]}\right) p\left(x_{1} \mid v_{2}\right)$.

\section{REFERENCES}

[1] S.-H. Lee and S.-Y. Chung, "A unified approach for network information theory," to appear in Proc. IEEE Int. Symp. Inform. Theory (ISIT), June 2015.

[2] H. H. J. Liao, "Multiple access channels," Ph.D. dissertation, University of Hawaii, Honolulu, HI, 1972.

[3] T. M. Cover and A. El Gamal, "Capacity theorems for the relay channel," IEEE Trans. Inf. Theory, vol. 25, pp. 572-584, Sep. 1979.

[4] D. Slepian and J. K. Wolf, "Noiseless coding of correlated information sources," IEEE Trans. Inf. Theory, vol. 19, pp. 471-480, Jul. 1973. 
[5] R. Ahlswede, N. Cai, S. R. Li, and R. W. Yeung, "Network information flow," IEEE Trans. Inf. Theory, vol. 46, pp. 1204-1216, July 2000.

[6] G. Kramer, M. Gastpar, and P. Gupta, "Cooperative strategies and capacity theorems for relay networks," IEEE Trans. Inf. Theory, vol. 51, pp. 3037-3063, Sep. 2005.

[7] A. S. Avestimehr, S. N. Diggavi, and D. Tse, "Wireless network information flow: A deterministic approach," IEEE Trans. Inf. Theory, vol. 57, pp. 1872-1905, April 2011.

[8] M. H. Yassaee and M. R. Aref, "Slepian-Wolf coding over cooperative relay networks," IEEE Trans. Inf. Theory, vol. 57, pp. 3462-3482, Jun. 2011.

[9] S. H. Lim, Y.-H. Kim, A. El Gamal, and S.-Y. Chung, "Noisy network coding," IEEE Trans. Inf. Theory, vol. 57, pp. 3132-3152, May 2011.

[10] J. Hou and G. Kramer, "Short message noisy network coding with a decode-forward option," IEEE Trans. Inf. Theory, submitted for publication. [Online]. Available: http://arxiv.org/abs/1304.1692

[11] S. Rini and A. Goldsmith, "A general approach to random coding for multi-terminal networks," in Proc. Information Theory and Applications Workshop, San Diego, USA, Feb. 2013.

[12] P. Minero, S. H. Lim, and Y.-H. Kim, “A unified approach to hybrid coding,” IEEE Trans. Inf. Theory, pp. 1509-1523, April 2015.

[13] S. H. Lim, K. T. Kim, and Y.-H. Kim, "Distributed decode-forward for multicast," in Proc. IEEE Int. Symp. Inform. Theory (ISIT), Jun.-Jul. 2014, pp. 636-640.

[14] — - "Distributed decode-forward for broadcast," in Proc. IEEE Information Theory Workshop (ITW), Hobart, TAS, November 2014, pp. 556-560.

[15] M. H. Yassaee, M. R. Aref, and A. Gohari, "Achievability proof via output statistics of random binning," IEEE Trans. Inf. Theory, vol. 60, pp. 6760-6786, Nov. 2014.

[16] B. Schein and R. Gallager, "The Gaussian parallel relay networks," in Proc. IEEE Int. Symp. Inform. Theory (ISIT), 2000, p. 22 .

[17] S. I. Gelfand and M. S. Pinsker, "Coding for channel with random parameters," Probl. Control Inf. Theory, vol. 9, pp. 19-31, 1980.

[18] K. Marton, "A coding theorem for the discrete memoryless broadcast channel," IEEE Trans. Inf. Theory, vol. 25, pp. 306-311, May 1979.

[19] T. S. Han and K. Kobayashi, "A new achievable rate region for the interference channel," IEEE Trans. Inf. Theory, vol. 27, pp. 49-60, Jan. 1981.

[20] H.-F. Chong, M. Motani, H. K. Garg, and H. El Gamal, "On the Han-Kobayashi region for the interference channel," IEEE Trans. Inf. Theory, vol. 54, pp. 3188-3195, Jul. 2008.

[21] T. Weissman, "Capacity of channels with action-dependent states," IEEE Trans. Inf. Theory, vol. 56, pp. 5396-5411, Nov. 2010 .

[22] B. Bandemer and A. El Gamal, "Interference decoding for deterministic channels," IEEE Trans. Inf. Theory, vol. 57, pp. 2966-2975, May 2011.

[23] — - "An achievable rate region for the 3-user-pair deterministic interference channel," in Proc. 49th Annual Allerton Conference on Communication, Control, and Computing, Allerton House, UIUC, Illinois, USA, Sep. 2011, pp. 38-44.

[24] T. M. Cover and C. Leung, "An achievable rate region for the multiple-access channel with feedback," IEEE Trans. Inf. Theory, vol. 27, pp. 292-298, May 1981. 
[25] L. Sankar, G. Kramer, and N. B. Mandayam, “Offset encoding for multiple-access relay channels," IEEE Trans. Inf. Theory, vol. 53, pp. 3814-3821, Oct. 2007.

[26] A. D. Wyner and J. Ziv, "The rate-distortion function for source coding with side information at the decoder," IEEE Trans. Inf. Theory, vol. 22, pp. 1-10, Jan. 1976.

[27] T. Berger, "Multiterminal source coding," The Information Theory Approach to Communications, New York: SpringerVerlag, 1977.

[28] S.-Y. Tung, "Multiterminal source coding," Ph.D. dissertation, Cornell University, Ithaca, NY, 1978.

[29] Z. Zhang and T. Berger, "New results in binary multiple descriptions," IEEE Trans. Inf. Theory, vol. 33, pp. 502-521, Jul. 1987.

[30] T. Cover, A. El Gamal, and M. Salehi, "Multiple access channels with arbitrarily correlated sources," IEEE Trans. Inf. Theory, vol. 26, pp. 648-657, Nov. 1980.

[31] T. S. Han and M. M. H. Costa, "Broadcast channels with arbitrarily correlated sources," IEEE Trans. Inf. Theory, vol. 33, pp. 641-650, Sep. 1987.

[32] W. Liu and B. Chen, "Interference channels with arbitrarily correlated sources," IEEE Trans. Inf. Theory, vol. 57, pp. 8027-8037, Dec. 2011.

[33] _ _ "Communicating correlated sources over interference channels: The lossy case," in Proc. IEEE Int. Symp. Inform. Theory (ISIT), Austin, Texas, 2010, pp. 345-349.

[34] P. Kocher, J. Jaffe, and B. Jun, “Differential power analysis,” vol. 28, pp. 803-807, Sep. 1982.

[35] P. Cuff, H.-I. Su, and A. El Gamal, "Cascade multiterminal source coding," in Proc. IEEE Int. Symp. Inform. Theory (ISIT), Seoul, South Korea, Jun.-Jul. 2009, pp. 1199-1203.

[36] S.-H. Lee and S.-Y. Chung, "Noisy network coding with partial DF," to appear in Proc. IEEE Int. Symp. Inform. Theory (ISIT), June 2015. [Online]. Available: http://arxiv.org/abs/1505.05435

[37] T. M. Cover, "Broadcast channels," IEEE Trans. Inf. Theory, vol. 18, pp. 2-14, Jan. 1972.

[38] C. Nair and A. El Gamal, "The capacity region of a class of three-receiver broadcast channels with degraded message sets," IEEE Trans. Inf. Theory, vol. 55, pp. 4479-4493, Oct. 2009.

[39] B. Bandemer, A. El Gamal, and Y.-H. Kim, "Simultaneous nonunique decoding is rate-optimal," in Proc. 50th Annual Allerton Conference on Communication, Control, and Computing, Monticello, IL, Oct. 2012.

[40] A. El Gamal and E. C. van der Meulen, "A proof of Marton's coding theorem for the discrete memoryless broadcast channel," IEEE Trans. Inf. Theory, vol. 27, pp. 120-122, Jan. 1981.

[41] A. El Gamal and Y.-H. Kim, Network information theory. Cambridge, U.K.: Cambridge Univ. Press, 2011.

[42] C. E. Shannon, "Channels with side information at the transmitter," IBM J. Res. Develop., vol. 2, pp. $289-293$, 1958.

[43] L. Wang and M. Naghshvar, "On the capacity of the noncausal relay channel," IEEE Trans. Inf. Theory, submitted for publication. [Online]. Available: http://arxiv.org/abs/1103.4177

[44] A. Lapidoth and S. Tinguely, "Sending a bivariate Gaussian over a Gaussian MAC," IEEE Trans. Inf. Theory, vol. 56, pp. 2714-2752, Jun. 2010.

[45] A. Orlitsky and J. R. Roche, “Coding for computing,” IEEE Trans. Inf. Theory, vol. 47, pp. 903-917, Mar. 2001.

[46] C. E. Shannon, “A mathematical theory of communication,” Bell Syst. Tech. J., vol. 27, pp. 379-423 and 623-656, 1948.

[47] A. El Gamal, N. Hassanpour, and J. Mammen, "Relay networks with delays," IEEE Trans. Inf. Theory, vol. 53, pp. 3413-3431, Oct. 2007. 
[48] T. M. Cover and A. El Gamal, "Achievable rates for multiple descriptions," IEEE Trans. Inf. Theory, vol. 28, pp. 851-857, Nov. 1982.

[49] P. G. J. Körner, “Common information is far less than mutual information,” Probl. Control Inf. Theory, vol. 2, pp. 149-162, 1973.

[50] W. Witsenhausen, "On sequences of pairs of dependent random variables," SIAM J. Appl. Math, vol. 28, pp. 100-113, 1975.

[51] I. Csiszár and J. Körner, "Broadcast channels with confidential messages," IEEE Trans. Inf. Theory, vol. 24, pp. 339-348, May 1978.

[52] B. E. Schein, "Distributed coordination in network information theory," Ph.D. dissertation, Massachusetts Institute of Technology, 2001.

[53] Y. Liang, "Multiuser communications with relaying and user cooperation," Ph.D. dissertation, Univ. Illinois at UrbanaChampaign, Urbana, IL, 2005.

[54] R. J. McEliece, The theory of information and coding. Addison-Wesley, Reading, 1977. 